\title{
Copper-Catalyzed Amino-Oxymethylation of Ynamides with
}

\section{N,O-Acetals}

\author{
Jianliang Yu, Guangyang Xu, Shengbiao Tang, Ying Shao and Jiangtao Sun* \\ Jiangsu Key Laboratory of Advanced Catalytic Materials \& Technology, School of Petrochemical \\ Engineering, Changzhou University, 1 Gehu Road, 213164 Changzhou, China \\ E-mail: jtsun@cczu.edu.cn or jtsun08@gmail.com
}

Table of Contents

1. General information

2. Preparation of substrates

3. General procedure for Scheme 2 and Scheme 3

4. Preparation of 3aa in $2 \mathrm{mmol}$ scale

5. Control experiments for Scheme 4

6. X-ray structure of 3ac and 3am

7. References

8. ${ }^{1} \mathrm{H}$ NMR, ${ }^{13} \mathrm{C}$ NMR and ${ }^{19} \mathrm{~F}$ NMR Spectra of compounds 


\section{General information}

All of the reactions were carried out in flame-dried tubes under an argon atmosphere. Solvents were dried prior to use. Commercially obtained reagents were used as received. Analytical thin layer chromatography (TLC) was carried out using pre-coated $(0.20 \mathrm{~mm}$ thickness) silica gel plates with $\mathrm{F}_{254}$ indicator. For column chromatography, 200-300 mesh basic aluminium oxide was used. ${ }^{1} \mathrm{H}$ NMR spectra were recorded on Bruker $300 \mathrm{MHz}$ or $400 \mathrm{MHz}$ spectrometer in DMSO- $d_{6}$. ${ }^{13} \mathrm{C}$ NMR spectra were recorded on Bruker $75 \mathrm{MHz}$ or $100 \mathrm{MHz}$ spectrometer in DMSO- $d_{6} \cdot{ }^{19} \mathrm{~F}$ NMR spectra were recorded on Bruker $376 \mathrm{MHz}$ spectrometer in DMSO- $d_{6}$. Data for ${ }^{1} \mathrm{H}$ NMR spectra were reported relative to DMSO- $d_{6}$ as an internal standard $(2.50 \mathrm{ppm})$ and were reported as follows: chemical shift $(\delta \mathrm{ppm})$, multiplicity, coupling constant $(\mathrm{Hz})$ and integration. Multiplicities are denoted as follows: $\mathrm{s}=$ singlet, $\mathrm{d}=$ doublet, $\mathrm{t}=$ triplet, $\mathrm{q}=$ quartet, $\mathrm{dd}=$ doublet of doublets, $\mathrm{td}=$ triplet of doublets, and $\mathrm{m}=$ multiplet. Data for ${ }^{13} \mathrm{C}$ NMR spectra were reported relative to DMSO- $d_{6}$ as an internal standard $(39.52 \mathrm{ppm})$ and were reported in terms of chemical shift $(\delta \mathrm{ppm})$. High resolution mass spectra (HRMS) were performed on Agilent 6540 Q-TOF mass spectrometer (ESI). Melting points were determined on a SGW X-4B melting point apparatus.

\section{Preparation of substrates}

$\mathrm{N}, \mathrm{O}$-acetals 1 were known compounds and prepared according to the literature procedures. ${ }^{1}$

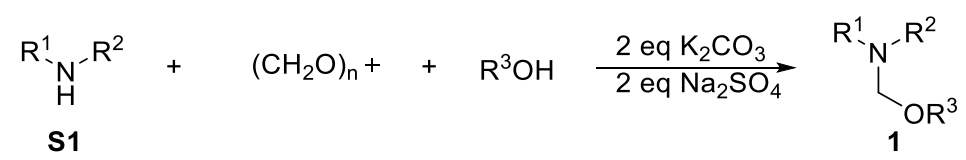

General procedure: To a solution of amine $\mathbf{S 1}(20 \mathrm{mmol})$ in $\mathrm{R}^{1} \mathrm{OH}(20 \mathrm{~mL})$ was added paraformaldehyde $(50 \mathrm{mmol}), \mathrm{K}_{2} \mathrm{CO}_{3}(40 \mathrm{mmol})$ and $\mathrm{Na}_{2} \mathrm{SO}_{4}(40 \mathrm{mmol})$ sequentially. The mixture was stirred at $\mathrm{rt}$ for $12 \mathrm{~h}$, then filtered and washed with $\mathrm{CH}_{2} \mathrm{Cl}_{2}\left(20 \mathrm{~mL}^{*} 3\right)$, the filtrate was concentrated under vacuum to give a mixture of oil and solid (paraformaldehyde). The mixture was dissolved in $\mathrm{Et}_{2} \mathrm{O}(50 \mathrm{~mL})$, filtered through a pad of celite and anhydrous $\mathrm{Na}_{2} \mathrm{SO}_{4}$, washed with $\mathrm{Et}_{2} \mathrm{O}(10 \mathrm{~mL} * 3)$. The combined filtrates were concentrated to about $20 \mathrm{~mL}$, filtered through a pad of celite and anhydrous $\mathrm{Na}_{2} \mathrm{SO}_{4}$ again, washed with $\mathrm{Et}_{2} \mathrm{O}(10 \mathrm{~mL} * 3)$ and concentrated give $\mathrm{N}, \mathrm{O}$-acetals 1, which was pure enough and used directly.

Yanmides 2 were known compounds and prepared according to the literature procedures. ${ }^{2}$

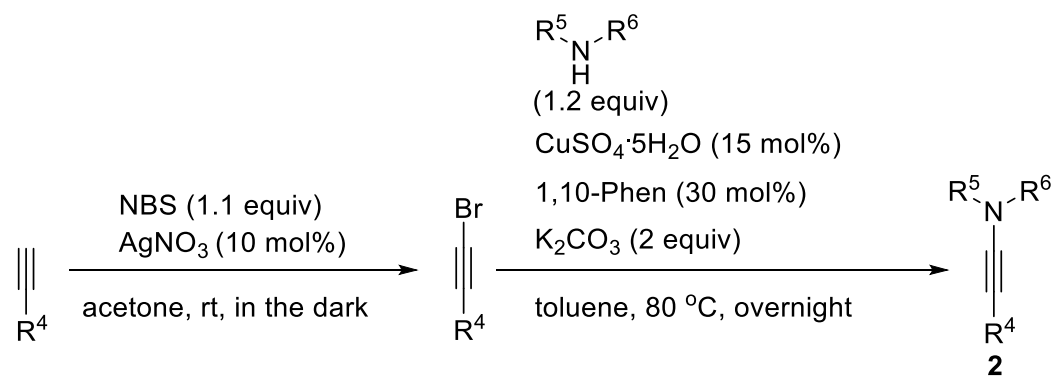

Step (1): In the dark, to a solution of terminal alkyne $(5.5 \mathrm{mmol})$ in $25 \mathrm{~mL}$ acetone was added NBS (1.1 g, $6.05 \mathrm{mmol})$ and $\mathrm{AgNO}_{3}(94 \mathrm{mg}, 0.55 \mathrm{mmol})$. The resulting mixture was stirred at room temperature for $3 \mathrm{~h}$. The mixture was filtered and washed with petroleum ether; the filtrate was evaporated. Then $20 \mathrm{~mL}$ petroleum ether was added to the residue, filtered again and the 
filtrate was evaporated under vacuum to give the corresponding alkynyl bromide which was used directly.

Step (2): Amide (5.0 mmol), alkynyl bromide $(5.5 \mathrm{mmol})$, copper sulfate pentahydrate $(187.0 \mathrm{mg}$, $0.75 \mathrm{mmol}), 1,10$-phenanthroline (270.0 mg, $1.5 \mathrm{mmol}), \mathrm{K}_{2} \mathrm{CO}_{3}(1.38 \mathrm{~g}, 10.0 \mathrm{mmol})$, and toluene $(25 \mathrm{~mL})$ were added to a flask. The reaction flask was evacuated under vacuum and flushed with nitrogen for three times. Then, the reaction mixture was heated to $80{ }^{\circ} \mathrm{C}$ and stirred overnight. The reaction mixture was cooled down to room temperature, filtered through a pad of silica gel, the filtrate was evaporated and purified by column chromatography (silica gel, EtOAc: Petroleum ether $=1: 20$ to $1: 10$ ) to give the desired ynamide 2 in high yield.

\section{General procedure for Scheme 2 and Scheme 3}

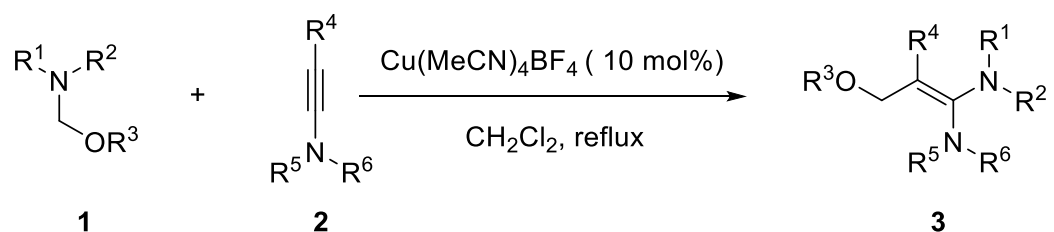

To a tube was added $\mathrm{Cu}(\mathrm{MeCN})_{4} \mathrm{BF}_{4}(0.1 \mathrm{mmol}, 3.2 \mathrm{mg}), 1(0.1 \mathrm{mmol}), 2(0.12 \mathrm{mmol})$ and $\mathrm{CH}_{2} \mathrm{Cl}_{2}(2 \mathrm{~mL})$ under an argon atmosphere. Then, the mixture was stirred at reflux for $6 \mathrm{~h}$. The reaction mixture was concentrated under vacuum, the crude residue was purified by column chromatography (basic aluminium oxide, EtOAc:Petroleum ether $=1: 50$ to 1:5) to give products 3.

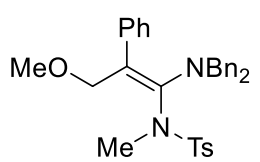

(Z)-N-(1-(dibenzylamino)-3-methoxy-2-phenylprop-1-en-1-yl)-N,4-dimethylbenzenesulfonamid (3aa):

Obtained as colorless oil after column chromatography (EtOAc:Petroleum ether $=1: 50$ to 1:20), $45.3 \mathrm{mg}, 86 \%$ yield. ${ }^{1} \mathrm{H}$ NMR (400MHz, DMSO- $\left.d_{6}\right) \delta 7.81(\mathrm{~d}, J=8.2 \mathrm{~Hz}, 2 \mathrm{H}), 7.43(\mathrm{~d}, J=8.2 \mathrm{~Hz}$, 2H), 7.39-7.28 (m, 6H), $7.17(\mathrm{~d}, J=6.9 \mathrm{~Hz}, 4 \mathrm{H}), 7.11-7.03(\mathrm{~m}, 3 \mathrm{H}), 6.86-6.79(\mathrm{~m}, 2 \mathrm{H}), 3.81(\mathrm{~d}, J$ $=10.9 \mathrm{~Hz}, 1 \mathrm{H}), 3.76-3.67(\mathrm{~m}, 3 \mathrm{H}), 3.40(\mathrm{~d}, J=14.6 \mathrm{~Hz}, 2 \mathrm{H}), 3.02(\mathrm{~s}, 3 \mathrm{H}), 2.94(\mathrm{~s}, 3 \mathrm{H}), 2.40(\mathrm{~s}$, $3 \mathrm{H}) .{ }^{13} \mathrm{C}$ NMR $\left(100 \mathrm{MHz}\right.$, DMSO- $\left.d_{6}\right) \delta 144.9,144.3,140.1,137.2,136.0,130.3,129.6,128.9$, 128.8, 128.3, 128.0, 127.7, 126.4, 115.0, 73.7, 57.8, 53.4, 39.0, 21.5. HRMS (ESI) calculated for $\mathrm{C}_{32} \mathrm{H}_{35} \mathrm{~N}_{2} \mathrm{O}_{3} \mathrm{~S}[\mathrm{M}+\mathrm{H}]^{+}:$527.2363, found: 527.2367 .<smiles>COC(=C(N(C)C)N(C)C)c1ccc(OC)cc1</smiles>

(Z)-N-(1-(dibenzylamino)-3-methoxy-2-(4-methoxyphenyl)prop-1-en-1-yl)-N,4-dimethylbenzene sulfonamide (3ab): 
Obtained as colorless oil after column chromatography (EtOAc:Petroleum ether $=1: 50$ to 1:20), $48.4 \mathrm{mg}, 87 \%$ yield. ${ }^{1} \mathrm{H}$ NMR (400 MHz, DMSO- $\left.d_{6}\right) \delta 7.78(\mathrm{~d}, J=8.2 \mathrm{~Hz}, 2 \mathrm{H}), 7.42(\mathrm{~d}, J=8.2$ $\mathrm{Hz}, 2 \mathrm{H}), 7.39-7.28(\mathrm{~m}, 6 \mathrm{H}), 7.17(\mathrm{~d}, J=7.0 \mathrm{~Hz}, 4 \mathrm{H}), 6.76(\mathrm{~d}, J=8.6 \mathrm{~Hz}, 2 \mathrm{H}), 6.63(\mathrm{~d}, J=8.6 \mathrm{~Hz}$, 2H), 3.76-3.66 (m , 7H), $3.41(\mathrm{~d}, J=14.6 \mathrm{~Hz}, 2 \mathrm{H}), 2.97(\mathrm{~s}, 3 \mathrm{H}), 2.92(\mathrm{~s}, 3 \mathrm{H}), 2.39$ (s, 3H). ${ }^{13} \mathrm{C}$ NMR (75 MHz, DMSO- $\left.d_{6}\right) \delta$ 158.0, 144.2, 137.4, 136.1, 132.2, 130.2, 129.9, 129.5, 128.8, 127.9, 127.7, 115.0, 113.7, 73.8, 57.8, 55.4, 53.3, 38.9, 21.5. HRMS (ESI) calculated for $\mathrm{C}_{33} \mathrm{H}_{37} \mathrm{~N}_{2} \mathrm{O}_{4} \mathrm{~S}$ $[\mathrm{M}+\mathrm{H}]^{+}: 557.2469$, found: 557.2467

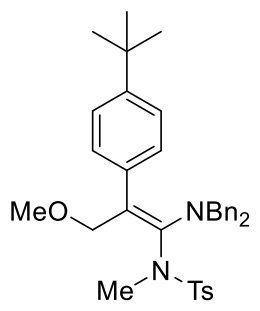

(Z)-N-(2-(4-(tert-butyl)phenyl)-1-(dibenzylamino)-3-methoxyprop-1-en-1-yl)-N,4-dimethylbenze nesulfonamide (3ac):

Obtained as a white solid after column chromatography (EtOAc:Petroleum ether $=1: 50$ to 1:20), $51.3 \mathrm{mg}, 88 \%$ yield, mp: $130-132{ }^{\circ} \mathrm{C} .{ }^{1} \mathrm{H}$ NMR $\left(400 \mathrm{MHz}, \mathrm{DMSO}-d_{6}\right) \delta 7.84(\mathrm{~d}, J=8.2 \mathrm{~Hz}, 2 \mathrm{H})$, $7.47(\mathrm{~d}, J=8.2 \mathrm{~Hz}, 2 \mathrm{H}), 7.39-7.29(\mathrm{~m}, 6 \mathrm{H}), 7.20(\mathrm{~d}, J=8.3 \mathrm{~Hz}, 2 \mathrm{H}), 7.11(\mathrm{~d}, J=6.8 \mathrm{~Hz}, 4 \mathrm{H})$, $6.93(\mathrm{~d}, J=8.3 \mathrm{~Hz}, 2 \mathrm{H}), 3.86(\mathrm{~d}, J=14.5 \mathrm{~Hz}, 2 \mathrm{H}), 3.70(\mathrm{~d}, J=10.8 \mathrm{~Hz}, 1 \mathrm{H}), 3.63-3.52(\mathrm{~m}, 3 \mathrm{H})$, $2.92(\mathrm{~s}, 3 \mathrm{H}), 2.87$ (s, , 3H), 2.43 (s, 3H), 1.27 (s, 9H). ${ }^{13} \mathrm{C}$ NMR (100 MHz, DMSO- $\left.d_{6}\right) \delta 148.9$, 145.2, 144.3, 137.8, 137.2, 135.8, 130.3, 129.4, 128.7, 128.6, 127.8, 125.1, 116.7, 73.5, 57.8, 54.1, 39.0, 34.5, 31.6, 21.5. HRMS (ESI) calculated for $\mathrm{C}_{36} \mathrm{H}_{43} \mathrm{~N}_{2} \mathrm{O}_{3} \mathrm{~S}[\mathrm{M}+\mathrm{H}]^{+}$: 583.2989, found: 583.2991 .<smiles>COCC(=C(N(C)C)N(C)C)c1ccc(Cl)cc1</smiles>

(Z)-N-(2-(4-chlorophenyl)-1-(dibenzylamino)-3-methoxyprop-1-en-1-yl)-N,4-dimethylbenzenesu lfonamide (3ad):

Obtained as colorless oil after column chromatography (EtOAc:Petroleum ether $=1: 50$ to 1:20), $46.0 \mathrm{mg}, 82 \%$ yield. ${ }^{1} \mathrm{H}$ NMR (400 MHz, DMSO- $\left.d_{6}\right) \delta 7.81(\mathrm{~d}, J=8.1 \mathrm{~Hz}, 2 \mathrm{H}), 7.43(\mathrm{~d}, J=8.1$ $\mathrm{Hz}, 2 \mathrm{H}), 7.41-7.31(\mathrm{~m}, 6 \mathrm{H}), 7.21(\mathrm{~d}, J=7.1 \mathrm{~Hz}, 4 \mathrm{H}), 7.08(\mathrm{~d}, J=8.3 \mathrm{~Hz}, 2 \mathrm{H}), 6.79(\mathrm{~d}, J=8.3 \mathrm{~Hz}$, 2H), $3.80(\mathrm{~d}, J=11.1 \mathrm{~Hz}, 1 \mathrm{H}), 3.75-3.67(\mathrm{~m}, 3 \mathrm{H}), 3.43(\mathrm{~d}, J=14.6 \mathrm{~Hz}, 2 \mathrm{H}), 3.03(\mathrm{~s}, 3 \mathrm{H}), 2.95(\mathrm{~s}$, $3 \mathrm{H}), 2.40(\mathrm{~s}, 3 \mathrm{H}) .{ }^{13} \mathrm{C}$ NMR $\left(100 \mathrm{MHz}\right.$, DMSO- $\left.d_{6}\right) \delta 145.2,144.4,139.0,136.9,135.9,130.8$, $130.4,130.3,129.6,129.0,128.2,128.1,127.8,113.2,73.4,57.8,53.4,38.9,21.5$. HRMS (ESI) calculated for $\mathrm{C}_{32} \mathrm{H}_{34} \mathrm{ClN}_{2} \mathrm{O}_{3} \mathrm{~S}[\mathrm{M}+\mathrm{H}]^{+}$: 561.1973, found: 561.1972 . 


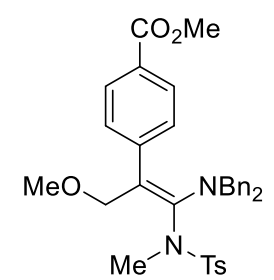

Methyl(Z)-4-(1-(dibenzylamino)-1-((N,4-dimethylphenyl)sulfonamido)-3-methoxyprop-1-en-2-y l)benzoate (3ae):

Obtained as colorless oil after column chromatography (EtOAc:Petroleum ether $=1: 50$ to 1:20), $47.4 \mathrm{mg}, 81 \%$ yield. ${ }^{1} \mathrm{H}$ NMR (400 MHz, DMSO- $\left.d_{6}\right) \delta 7.82(\mathrm{~d}, J=8.3 \mathrm{~Hz}, 2 \mathrm{H}), 7.60$ (d, $J=8.5$ $\mathrm{Hz}, 2 \mathrm{H}), 7.44-7.32(\mathrm{~m}, 8 \mathrm{H}), 7.23(\mathrm{~d}, J=6.8 \mathrm{~Hz}, 4 \mathrm{H}), 6.88(\mathrm{~d}, J=8.3 \mathrm{~Hz}, 2 \mathrm{H}), 3.91(\mathrm{~d}, J=11.1$ $\mathrm{Hz}, 1 \mathrm{H}), 3.84-3.79(\mathrm{~m}, 4 \mathrm{H}), 3.69(\mathrm{~d}, J=14.5 \mathrm{~Hz}, 2 \mathrm{H}), 3.44-3.39(\mathrm{~m}, 2 \mathrm{H}), 3.12(\mathrm{~s}, 3 \mathrm{H}), 2.97(\mathrm{~s}$, $3 \mathrm{H}), 2.37$ (s, 3H). ${ }^{13} \mathrm{C}$ NMR (75 MHz, DMSO- $\left.d_{6}\right) \delta 166.5,145.9,145.5,144.5,136.5,135.8$, 130.3, 129.7, 129.1, 129.0, 128.4, 128.2 127.8, 127.0, 112.0, 73.2, 57.8, 53.3, 52.4, 39.0, 21.5. HRMS (ESI) calculated for $\mathrm{C}_{34} \mathrm{H}_{37} \mathrm{~N}_{2} \mathrm{O}_{5} \mathrm{~S}[\mathrm{M}+\mathrm{H}]^{+}: 585.2418$, found: 585.2421.

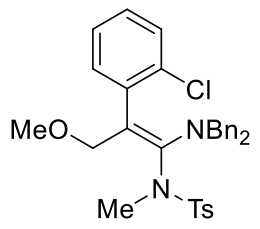

(Z)-N-(2-(2-chlorophenyl)-1-(dibenzylamino)-3-methoxyprop-1-en-1-yl)-N,4-dimethylbenzenesu lfonamide (3af):

Obtained as colorless oil after column chromatography (EtOAc:Petroleum ether $=1: 50$ to 1:20), $42.1 \mathrm{mg}, 75 \%$ yield. ${ }^{1} \mathrm{H}$ NMR (400 MHz, DMSO- $\left.d_{6}\right) \delta 7.79(\mathrm{~d}, J=8.2 \mathrm{~Hz}, 2 \mathrm{H}), 7.48(\mathrm{~d}, J=8.2$ $\mathrm{Hz}, 2 \mathrm{H}), 7.38-7.19(\mathrm{~m}, 8 \mathrm{H}), 7.14-7.08(\mathrm{~m}, 1 \mathrm{H}), 7.05(\mathrm{~d}, J=7.0 \mathrm{~Hz}, 4 \mathrm{H}), 6.65-6.50(\mathrm{~m}, 1 \mathrm{H}), 4.00$ $(\mathrm{d}, J=14.6 \mathrm{~Hz}, 2 \mathrm{H}), 3.52(\mathrm{~d}, J=11.0 \mathrm{~Hz}, 1 \mathrm{H}), 3.42(\mathrm{~d}, J=14.6 \mathrm{~Hz}, 2 \mathrm{H}), 3.15(\mathrm{~d}, J=11.0 \mathrm{~Hz}$, 1H), $2.81(\mathrm{~s}, 3 \mathrm{H}), 2.78(\mathrm{~s}, 3 \mathrm{H}), 2.42(\mathrm{~s}, 3 \mathrm{H}) .{ }^{13} \mathrm{C}$ NMR (100 MHz, DMSO- $\left.d_{6}\right) \delta$ 147.5, 144.6, 137.9, 137.86, 135.2, 133.3, 133.2, 130.4, 130.2, 129.2, 129.1, 128.8, 127.74, 127.73, 127.1, 117.2, 70.9, 58.2, 54.1, 39.8, 21.5. HRMS (ESI) calculated for $\mathrm{C}_{32} \mathrm{H}_{34} \mathrm{ClN}_{2} \mathrm{O}_{3} \mathrm{~S}[\mathrm{M}+\mathrm{H}]^{+}: 561.1973$, found: 561.1977 .

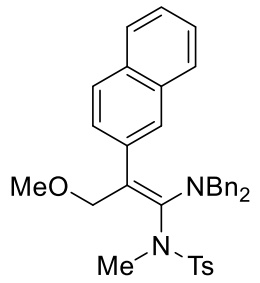

(Z)-N-(1-(dibenzylamino)-3-methoxy-2-(naphthalen-2-yl)prop-1-en-1-yl)-N,4-dimethylbenzenes ulfonamide (3ag):

Obtained as colorless oil after column chromatography (EtOAc:Petroleum ether $=1: 50$ to 1:20), $46.7 \mathrm{mg}, 81 \%$ yield. ${ }^{1} \mathrm{H}$ NMR (400 MHz, DMSO- $\left.d_{6}\right) \delta 7.84(\mathrm{~d}, J=8.3 \mathrm{~Hz}, 2 \mathrm{H}), 7.79-7.74(\mathrm{~m}, 1 \mathrm{H})$, $7.62(\mathrm{~d}, J=8.5 \mathrm{~Hz}, 1 \mathrm{H}), 7.44-7.32(\mathrm{~m}, 11 \mathrm{H}), 7.24-7.18(\mathrm{~m}, 4 \mathrm{H}), 7.18-7.13(\mathrm{~m}, 2 \mathrm{H}), 4.02(\mathrm{~d}, J=$ $11.0 \mathrm{~Hz}, 1 \mathrm{H}), 3.92(\mathrm{~d}, J=11.0 \mathrm{~Hz}, 1 \mathrm{H}), 3.70(\mathrm{~d}, J=14.5 \mathrm{~Hz}, 2 \mathrm{H}), 3.40-3.35(\mathrm{~m}, 2 \mathrm{H}), 3.18(\mathrm{~s}, 3 \mathrm{H})$, 
$3.00(\mathrm{~s}, 3 \mathrm{H}), 2.37(\mathrm{~s}, 3 \mathrm{H}) .{ }^{13} \mathrm{C}$ NMR (75 MHz, DMSO- $\left.d_{6}\right) \delta 145.1,144.3,140.0,137.7,137.0$, 136.1, 133.1, 131.8, 130.3, 130.2, 129.8, 129.0, 128.1, 127.7, 127.6, 127.3, 126.5, 126.3, 125.8, 113.5, 73.8, 57.9, 53.1, 39.1, 21.5. HRMS (ESI) calculated for $\mathrm{C}_{36} \mathrm{H}_{37} \mathrm{~N}_{2} \mathrm{O}_{3} \mathrm{~S}[\mathrm{M}+\mathrm{H}]^{+}: 577.2519$, found: 577.2524 .

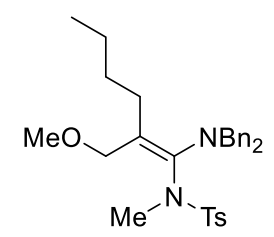

(Z)-N-(1-(dibenzylamino)-2-(methoxymethyl)hex-1-en-1-yl)-N,4-dimethylbenzenesulfonamide (3ah):

Obtained as colorless oil after column chromatography (EtOAc:Petroleum ether $=1: 50$ to 1:20), $44.6 \mathrm{mg}, 88 \%$ yield. ${ }^{1} \mathrm{H}$ NMR (300 MHz, DMSO- $\left.d_{6}\right) \delta 7.72(\mathrm{~d}, J=8.1 \mathrm{~Hz}, 2 \mathrm{H}), 7.45$ (d, $J=8.1$ $\mathrm{Hz}, 2 \mathrm{H}), 7.40-7.22(\mathrm{~m}, 10 \mathrm{H}), 3.42-3.04(\mathrm{~m}, 7 \mathrm{H}), 3.00(\mathrm{~s}, 3 \mathrm{H}), 2.83-2.64(\mathrm{~m}, 2 \mathrm{H}), 2.44(\mathrm{~s}, 3 \mathrm{H})$, $2.22(\mathrm{t}, J=7.4 \mathrm{~Hz} 2 \mathrm{H}), 1.26-1.12(\mathrm{~m}, 2 \mathrm{H}), 1.10-0.96(\mathrm{~m}, 2 \mathrm{H}), 0.78(\mathrm{t}, J=7.2 \mathrm{~Hz}, 3 \mathrm{H}) .{ }^{13} \mathrm{C}$ NMR $\left(75 \mathrm{MHz}\right.$, DMSO- $\left.d_{6}\right) \delta 145.3,144.1,139.9,136.3,130.2,129.0,128.6,127.8,127.4,121.0,58.1$, 57.4, 52.6, 37.5, 29.6, 26.6, 22.5, 21.5, 14.3. HRMS (ESI) calculated for $\mathrm{C}_{30} \mathrm{H}_{39} \mathrm{~N}_{2} \mathrm{O}_{3} \mathrm{~S}[\mathrm{M}+\mathrm{H}]^{+}$: 507.2676, found: 507.2679.

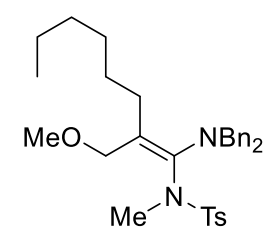

(Z)-N-(1-(dibenzylamino)-2-(methoxymethyl)oct-1-en-1-yl)-N,4-dimethylbenzenesulfonamide

(3ai):

Obtained as colorless oil after column chromatography (EtOAc:Petroleum ether $=1: 50$ to 1:20), $45.5 \mathrm{mg}, 85 \%$ yield. ${ }^{1} \mathrm{H}$ NMR (400 MHz, DMSO- $\left.d_{6}\right) \delta 7.69(\mathrm{~d}, J=8.3 \mathrm{~Hz}, 2 \mathrm{H}), 7.43$ (d, $J=8.3$ $\mathrm{Hz}, 2 \mathrm{H}), 7.36-7.28(\mathrm{~m}, 8 \mathrm{H}), 7.27-7.20(\mathrm{~m}, 2 \mathrm{H}), 3.65-3.35(\mathrm{~m}, 2 \mathrm{H}), 3.28-3.06(\mathrm{~m}, 5 \mathrm{H}), 2.99(\mathrm{~s}, 3 \mathrm{H})$, 2.85-2.55 (m, 2H), $2.43(\mathrm{~s}, 3 \mathrm{H}), 2.19(\mathrm{t}, J=7.3 \mathrm{~Hz}, 2 \mathrm{H}), 1.26-0.98(\mathrm{~m}, 8 \mathrm{H}), 0.84(\mathrm{t}, J=7.1 \mathrm{~Hz}$, $3 \mathrm{H}) .{ }^{13} \mathrm{C}$ NMR (75 MHz, DMSO- $\left.d_{6}\right) \delta 145.3,144.1,139.9,136.3,130.2,129.0,128.6,127.8$, $127.4,120.9,58.2,57.4,52.7,37.5,31.6,28.9,27.3,26.9,22.4,21.5$, 14.4. HRMS (ESI) calculated for $\mathrm{C}_{32} \mathrm{H}_{43} \mathrm{~N}_{2} \mathrm{O}_{3} \mathrm{~S}[\mathrm{M}+\mathrm{H}]^{+}$: 535.2989, found: 535.2985.

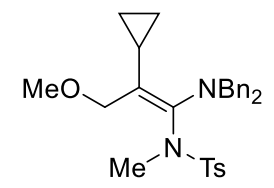

(Z)-N-(2-cyclopropyl-1-(dibenzylamino)-3-methoxyprop-1-en-1-yl)-N,4-dimethylbenzenesulfona mide (3aj):

Obtained as colorless oil after column chromatography (EtOAc:Petroleum ether $=1: 50$ to 1:20), $37.8 \mathrm{mg}, 77 \%$ yield. ${ }^{1} \mathrm{H}$ NMR (400 MHz, DMSO- $\left.d_{6}\right) \delta 7.68(\mathrm{~d}, J=8.2 \mathrm{~Hz}, 2 \mathrm{H}), 7.40(\mathrm{~d}, J=8.2$ $\mathrm{Hz}, 2 \mathrm{H}), 7.36(\mathrm{~d}, J=4.4 \mathrm{~Hz}, 8 \mathrm{H}), 7.31-7.24(\mathrm{~m}, 2 \mathrm{H}), 4.15(\mathrm{~d}, J=15.2 \mathrm{~Hz}, 2 \mathrm{H}), 4.04(\mathrm{~d}, J=15.2$ 
$\mathrm{Hz}, 2 \mathrm{H}), 3.15$ (d, $J=10.7 \mathrm{~Hz}, 1 \mathrm{H}), 2.96(\mathrm{~d}, J=10.7 \mathrm{~Hz}, 1 \mathrm{H}), 2.85$ (s, 3H), 2.40 (s, 3H), 2.39 (s, $3 \mathrm{H}), 1.72-1.63(\mathrm{~m}, 1 \mathrm{H}), 0.64-0.40(\mathrm{~m}, 4 \mathrm{H}) .{ }^{13} \mathrm{C}$ NMR $\left(75 \mathrm{MHz}, \mathrm{DMSO}-d_{6}\right) \delta 145.4,144.1,139.5$, 135.8, 130.1, 128.9, 128.3, 127.7, $127.5118 .8,69.2,57.6,54.4,38.6,21.5,13.5,5.8,5.7 . \mathrm{K}$ HRMS (ESI) calculated for $\mathrm{C}_{29} \mathrm{H}_{35} \mathrm{~N}_{2} \mathrm{O}_{3} \mathrm{~S}[\mathrm{M}+\mathrm{H}]^{+}:$491.2363, found: 491.2366 .

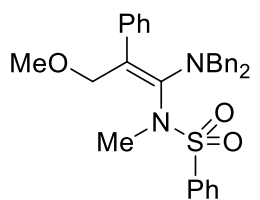

(Z)-N-(1-(dibenzylamino)-3-methoxy-2-phenylprop-1-en-1-yl)-N-methylbenzenesulfonamide (3ak):

Obtained as colorless oil after column chromatography (EtOAc:Petroleum ether $=1: 50$ to 1:20), $45.7 \mathrm{mg}, 89 \%$ yield. ${ }^{1} \mathrm{H}$ NMR (400 MHz, DMSO- $\left.d_{6}\right) \delta 7.93(\mathrm{~d}, J=7.8 \mathrm{~Hz}, 2 \mathrm{H}), 7.73$ (t, $J=7.3 \mathrm{~Hz}$ $1 \mathrm{H}), 7.63(\mathrm{t}, J=7.6 \mathrm{~Hz}, 2 \mathrm{H}), 7.40-7.28(\mathrm{~m}, 6 \mathrm{H}), 7.16(\mathrm{~d}, J=7.2 \mathrm{~Hz}, 4 \mathrm{H}), 7.12-7.04(\mathrm{~m}, 3 \mathrm{H}), 6.84$ $(\mathrm{d}, J=7.5 \mathrm{~Hz}, 2 \mathrm{H}), 3.83-3.72(\mathrm{~m}, 2 \mathrm{H}), 3.72-3.66(\mathrm{~m}, 2 \mathrm{H}), 3.41(\mathrm{~d}, J=14.5 \mathrm{~Hz}, 2 \mathrm{H}), 3.04(\mathrm{~s}, 3 \mathrm{H})$, $2.92(\mathrm{~s}, 3 \mathrm{H}) .{ }^{13} \mathrm{C}$ NMR $\left(100 \mathrm{MHz}, \mathrm{DMSO}-d_{6}\right) \delta 144.9,140.1,138.8,137.2,133.9,129.9,129.6$, $128.9,128.8,128.3,128.0,127.7,126.5,115.2,73.7,57.9,53.4,39.2$. HRMS (ESI) calculated for $\mathrm{C}_{31} \mathrm{H}_{33} \mathrm{~N}_{2} \mathrm{O}_{3} \mathrm{~S}[\mathrm{M}+\mathrm{H}]^{+}:$513.2206, found: 513.2210.

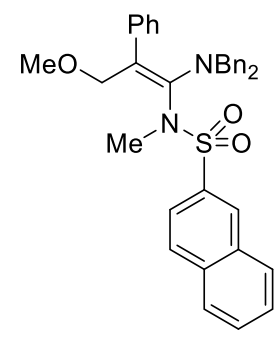

(Z)-N-(1-(dibenzylamino)-3-methoxy-2-phenylprop-1-en-1-yl)- $N$-methylnaphthalene-2-sulfona mide (3al):

Obtained as colorless oil after column chromatography (EtOAc:Petroleum ether $=1: 50$ to 1:20), $51.2 \mathrm{mg}, 91 \%$ yield. ${ }^{1} \mathrm{H}$ NMR (400 MHz, DMSO- $\left.d_{6}\right) \delta 8.57(\mathrm{~s}, 1 \mathrm{H}), 8.15(\mathrm{~d}, J=8.8 \mathrm{~Hz}, 1 \mathrm{H}), 8.08$ $(\mathrm{t}, J=8.5 \mathrm{~Hz}, 2 \mathrm{H}), 7.95(\mathrm{~d}, J=8.8 \mathrm{~Hz}, 1 \mathrm{H}), 7.75-7.65(\mathrm{~m}, 2 \mathrm{H}), 7.41-7.30(\mathrm{~m}, 6 \mathrm{H}), 7.19(\mathrm{~d}, J=7.1$ $\mathrm{Hz}, 4 \mathrm{H}), 7.12-7.02(\mathrm{~m}, 3 \mathrm{H}), 6.80(\mathrm{~d}, J=7.1 \mathrm{~Hz}, 2 \mathrm{H}), 3.84(\mathrm{q}, J=11.0 \mathrm{~Hz}, 2 \mathrm{H}), 3.68(\mathrm{~d}, J=14.5$ $\mathrm{Hz}, 2 \mathrm{H}), 3.35-3.29(\mathrm{~m}, 2 \mathrm{H}), 3.16(\mathrm{~s}, 3 \mathrm{H}), 2.87(\mathrm{~s}, 3 \mathrm{H}){ }^{13} \mathrm{C}$ NMR $\left(100 \mathrm{MHz}, \mathrm{DMSO}-d_{6}\right) \delta 145.6$, 144.9, 140.0, 137.1, 136.1, 134.9, 132.2, 129.9, 129.8, 129.7, 129.6, 128.9, 128.86, 128.7, 128.4, 128.3, 128.0, 126.5, 123.0, 115.0, 73.8, 57.8, 53.2, 39.4. HRMS (ESI) calculated for $\mathrm{C}_{35} \mathrm{H}_{35} \mathrm{~N}_{2} \mathrm{O}_{3} \mathrm{~S}$ $[\mathrm{M}+\mathrm{H}]^{+}:$: 563.2363, found: 563.2360 .<smiles>CCC/C(=C(\COC)c1ccccc1)N([N+](=O)[O-])S(=O)(=O)c1ccc([N+](=O)[O-])cc1</smiles> 
(Z)-N-(1-(dibenzylamino)-3-methoxy-2-phenylprop-1-en-1-yl)-N-methyl-4-nitrobenzenesulfona mide (3am):

Obtained as a yellow solid after column chromatography (EtOAc:Petroleum ether $=1: 20$ to 1:10), $41.3 \mathrm{mg}, 74 \%$ yield, mp: $153-155^{\circ} \mathrm{C} .{ }^{1} \mathrm{H}$ NMR $\left(400 \mathrm{MHz}, \mathrm{DMSO}-d_{6}\right) \delta 8.41(\mathrm{~d}, J=8.8 \mathrm{~Hz}, 2 \mathrm{H})$, $8.17(\mathrm{~d}, J=8.8 \mathrm{~Hz}, 2 \mathrm{H}), 7.41-7.30(\mathrm{~m}, 6 \mathrm{H}), 7.17(\mathrm{~d}, J=7.0 \mathrm{~Hz}, 4 \mathrm{H}), 7.14-7.03(\mathrm{~m}, 3 \mathrm{H}), 6.83$ (d, $J=6.9 \mathrm{~Hz}, 2 \mathrm{H}), 3.81(\mathrm{~d}, J=11.0 \mathrm{~Hz}, 1 \mathrm{H}), 3.75-3.63(\mathrm{~m}, 3 \mathrm{H}), 3.44-3.39(\mathrm{~m}, 2 \mathrm{H}), 3.13(\mathrm{~s}, 3 \mathrm{H})$, $2.93(\mathrm{~s}, 3 \mathrm{H}) .{ }^{13} \mathrm{C}$ NMR $\left(100 \mathrm{MHz}, \mathrm{DMSO}-d_{6}\right) \delta 150.5,144.2,144.0,139.8,137.0,129.6,129.3$, $128.9,128.8,128.3,128.0,125.1,115.8,73.6,58.0,53.4,39.5$. HRMS (ESI) calculated for $\mathrm{C}_{31} \mathrm{H}_{32} \mathrm{~N}_{3} \mathrm{O}_{5} \mathrm{~S}[\mathrm{M}+\mathrm{H}]^{+}:$558.2057, found: 558.2060.

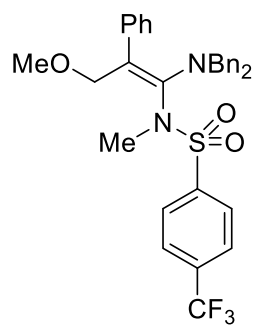

(Z)-N-(1-(dibenzylamino)-3-methoxy-2-phenylprop-1-en-1-yl)-N-methyl-4-(trifluoromethyl)benz enesulfonamide (3an):

Obtained as colorless oil after column chromatography (EtOAc:Petroleum ether $=1: 50$ to 1:20), $46.5 \mathrm{mg}, 80 \%$ yield. ${ }^{1} \mathrm{H}$ NMR (400 MHz, DMSO- $\left.d_{6}\right) \delta 8.15(\mathrm{~d}, J=8.4 \mathrm{~Hz}, 2 \mathrm{H}), 8.02(\mathrm{~d}, J=8.4$ $\mathrm{Hz}, 2 \mathrm{H}), 7.40-7.29(\mathrm{~m}, 6 \mathrm{H}), 7.17(\mathrm{~d}, J=7.0 \mathrm{~Hz}, 4 \mathrm{H}), 7.13-7.05(\mathrm{~m}, 3 \mathrm{H}), 6.89-6.82(\mathrm{~m}, 2 \mathrm{H})$, 3.79-3.69 (m, 3H), $3.63(\mathrm{~d}, J=10.9 \mathrm{~Hz}, 1 \mathrm{H}), 3.44$ (d, $J=14.6 \mathrm{~Hz}, 2 \mathrm{H}), 3.08(\mathrm{~s}, 3 \mathrm{H}), 2.89$ (s, 3H). ${ }^{13} \mathrm{C}$ NMR $\left(75 \mathrm{MHz}, \mathrm{DMSO}-d_{6}\right) \delta 144.3,142.5,139.8,137.1,133.9,133.6,133.2,132.9,129.5$, 128.9, 128.75, 128.7, 128.3, 128.0, 127.1, 127.0, 126.6, 125.2, 122.5, 115.7, 73.5, 57.8, 53.4, 39.3. ${ }^{19} \mathrm{~F}$ NMR (376 MHz, DMSO- $d_{6}$ ) $\delta$-61.7. HRMS (ESI) calculated for $\mathrm{C}_{32} \mathrm{H}_{32} \mathrm{~F}_{3} \mathrm{~N}_{2} \mathrm{O}_{3} \mathrm{~S}[\mathrm{M}+\mathrm{H}]^{+}$: 581.2080, found: 581.2078 .

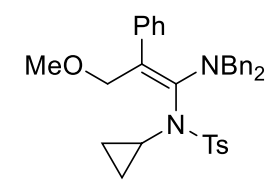

(Z)-N-cyclopropyl-N-(1-(dibenzylamino)-3-methoxy-2-phenylprop-1-en-1-yl)-4-methylbenzenes ulfonamide (3ao):

Obtained as colorless oil after column chromatography (EtOAc:Petroleum ether $=1: 50$ to 1:20), $36.0 \mathrm{mg}, 65 \%$ yield. ${ }^{1} \mathrm{H}$ NMR (400 MHz, DMSO- $\left.d_{6}\right) \delta 7.88(\mathrm{~d}, J=8.2 \mathrm{~Hz}, 2 \mathrm{H}), 7.46$ (d, $J=8.2$ $\mathrm{Hz}, 2 \mathrm{H}), 7.37-7.27(\mathrm{~m}, 6 \mathrm{H}), 7.18(\mathrm{~d}, J=6.7 \mathrm{~Hz}, 4 \mathrm{H}), 7.11-7.04(\mathrm{~m}, 5 \mathrm{H}), 3.93-3.83(\mathrm{~m}, 3 \mathrm{H}), 3.66$ $(\mathrm{d}, J=10.4 \mathrm{~Hz}, 1 \mathrm{H}), 3.46(\mathrm{~d}, J=14.8 \mathrm{~Hz}, 2 \mathrm{H}), 3.05(\mathrm{~s}, 3 \mathrm{H}), 2.74-2.67(\mathrm{~m}, 1 \mathrm{H}), 2.42(\mathrm{~s}, 3 \mathrm{H})$, $0.90-0.82(\mathrm{~m}, 1 \mathrm{H}), 0.80-0.70(\mathrm{~m}, 2 \mathrm{H}), 0.60-0.50(\mathrm{~m}, 1 \mathrm{H}) .{ }^{13} \mathrm{C}$ NMR $\left(75 \mathrm{MHz}, \mathrm{DMSO}-d_{6}\right) \delta 144.6$, $142.9,140.9,136.9,136.3,130.1,129.5,128.7,128.6,128.5,128.2,127.9,126.2,114.0,74.7$, 57.9, 52.8, 30.3, 21.5, 9.3, 7.5. HRMS (ESI) calculated for $\mathrm{C}_{34} \mathrm{H}_{37} \mathrm{~N}_{2} \mathrm{O}_{3} \mathrm{~S}[\mathrm{M}+\mathrm{H}]^{+}: 553.2519$, found: 553.2523 . 


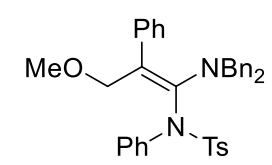

(Z)-N-(1-(dibenzylamino)-3-methoxy-2-phenylprop-1-en-1-yl)-4-methyl-N-phenylbenzenesulfon amide (3ap):

Obtained as colorless oil after column chromatography (EtOAc:Petroleum ether $=1: 50$ to 1:20), $45.4 \mathrm{mg}, 77 \%$ yield. ${ }^{1} \mathrm{H}$ NMR (400 MHz, DMSO- $\left.d_{6}\right) \delta$ 7.66-7.57 (m, 4H), 7.55-7.47 (m, 3H), 7.37 $(\mathrm{d}, J=8.1 \mathrm{~Hz}, 2 \mathrm{H}), 7.28-7.15(\mathrm{~m}, 6 \mathrm{H}), 7.08-7.01(\mathrm{~m}, 1 \mathrm{H}), 6.99-6.90(\mathrm{~m}, 4 \mathrm{H}), 6.74(\mathrm{~d}, J=7.2 \mathrm{~Hz}$, 4H), 4.26 (d, $J=10.4 \mathrm{~Hz}, 1 \mathrm{H}), 3.97(\mathrm{~d}, J=14.6 \mathrm{~Hz}, 2 \mathrm{H}), 3.88(\mathrm{~d}, J=10.4 \mathrm{~Hz}, 1 \mathrm{H}), 3.19$ (d, $J=$ $14.6 \mathrm{~Hz}, 2 \mathrm{H}), 3.08$ (s, 3H), 2.37 (s, 3H). ${ }^{13} \mathrm{C}$ NMR (75 MHz, DMSO- $\left.d_{6}\right) \delta 144.7,143.4,140.6$, $140.1,136.5,136.4,130.2,130.1,129.3,128.7,128.3,128.2,128.05,127.98,127.8,127.8,126.4$, 114.9, 74.7, 58.1, 52.5, 21.5. HRMS (ESI) calculated for $\mathrm{C}_{37} \mathrm{H}_{37} \mathrm{~N}_{2} \mathrm{O}_{3} \mathrm{~S}[\mathrm{M}+\mathrm{H}]^{+}: 589.2519$, found: 589.2522 .

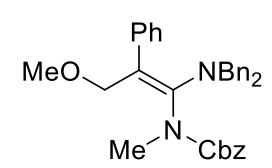

Benzyl (Z)-(1-(dibenzylamino)-3-methoxy-2-phenylprop-1-en-1-yl)(methyl)carbamate (3aq):

Obtained as colorless oil after column chromatography (EtOAc:Petroleum ether $=1: 50$ to 1:20), $34.0 \mathrm{mg}, 67 \%$ yield. ${ }^{1} \mathrm{H}$ NMR (400 MHz, DMSO- $\left.d_{6}\right) \delta$ 7.50-7.29 (m, 7H), 7.27-7.17(m, 5H), 7.11-7.00 (m, 6H), 7.00-6.88 (m, 2H), 5.26-4.98 (m, 2H), $4.03(\mathrm{~d}, J=11.2 \mathrm{~Hz}, 1 \mathrm{H}), 3.89-3.78(\mathrm{~m}$, $1 \mathrm{H}), 3.76-3.61(\mathrm{~m}, 2 \mathrm{H}), 3.54-3.37(\mathrm{~m}, 2 \mathrm{H}), 3.11(\mathrm{~s}, 3 \mathrm{H}), 3.04-2.88(\mathrm{~m}, 3 \mathrm{H}) .{ }^{13} \mathrm{C}$ NMR $(100 \mathrm{MHz}$, DMSO- $\left.d_{6}\right) \delta 156.0,144.4,140.7,137.5,136.6,129.3,128.8,128.73,128.71,128.5,128.2,127.8$, 126.2, 110.9, 74.2, 67.7, 57.5, 53.0, 36.0. HRMS (ESI) calculated for $\mathrm{C}_{33} \mathrm{H}_{35} \mathrm{~N}_{2} \mathrm{O}_{3}[\mathrm{M}+\mathrm{H}]^{+}$: 507.2642, found: 507.2639 .

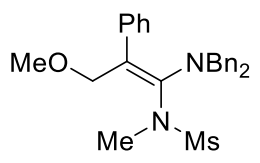

(Z)-N-(1-(dibenzylamino)-3-methoxy-2-phenylprop-1-en-1-yl)-N-methylmethanesulfonamide (3ar):

Obtained as colorless oil after column chromatography (EtOAc:Petroleum ether $=1: 20$ to 1:10), $32.5 \mathrm{mg}, 72 \%$ yield. ${ }^{1} \mathrm{H}$ NMR (400 MHz, DMSO- $\left.d_{6}\right) \delta 7.42-7.30(\mathrm{~m}, 6 \mathrm{H}), 7.23(\mathrm{~d}, J=6.8 \mathrm{~Hz}, 4 \mathrm{H})$, 7.09-7.03 (m, 1H), 7.03-6.96 (m, 2H), $6.73(\mathrm{~d}, J=7.3 \mathrm{~Hz}, 2 \mathrm{H}), 4.23-4.14(\mathrm{~m}, 2 \mathrm{H}), 3.73(\mathrm{~d}, J=$ $14.6 \mathrm{~Hz}, 2 \mathrm{H}), 3.40$ (d, J=14.6Hz, 2H), 3.21 (s, 3H), $3.18(\mathrm{~s}, 3 \mathrm{H}), 3.13(\mathrm{~s}, 3 \mathrm{H}) .{ }^{13} \mathrm{C} \mathrm{NMR}(75 \mathrm{MHz}$, DMSO- $\left.d_{6}\right) \delta 144.5,140.0,137.1,129.7,129.0,128.6,128.1,128.0,126.3,114.4,74.1,57.9,52.7$, 39.5, 38.4. HRMS (ESI) calculated for $\mathrm{C}_{26} \mathrm{H}_{31} \mathrm{~N}_{2} \mathrm{O}_{3} \mathrm{~S}[\mathrm{M}+\mathrm{H}]^{+}$: 451.2050, found: 451.2055 .

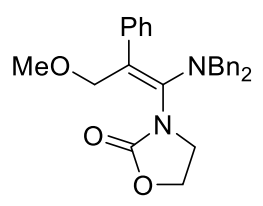

(Z)-3-(1-(dibenzylamino)-3-methoxy-2-phenylprop-1-en-1-yl)oxazolidin-2-one (3as): 
Obtained as colorless oil after column chromatography (EtOAc:Petroleum ether $=1: 10$ to 1:5), $35.6 \mathrm{mg}, 83 \%$ yield. ${ }^{1} \mathrm{H}$ NMR (400 MHz, DMSO- $\left.d_{6}\right) \delta 7.41-7.32(\mathrm{~m}, 6 \mathrm{H}), 7.19(\mathrm{~d}, J=6.6 \mathrm{~Hz}, 4 \mathrm{H})$, 7.10-7.05 (m, 1H), 6.99 (t, $J=7.2 \mathrm{~Hz}, 2 \mathrm{H}), 6.70(\mathrm{~d}, J=7.2 \mathrm{~Hz}, 2 \mathrm{H}), 4.49(\mathrm{t}, J=8.1 \mathrm{~Hz}, 2 \mathrm{H}), 4.20$ $(\mathrm{d}, J=11.8 \mathrm{~Hz}, 1 \mathrm{H}), 3.88(\mathrm{t}, J=9.7 \mathrm{~Hz}, 2 \mathrm{H}), 3.80-3.68(\mathrm{~m}, 3 \mathrm{H}), 3.42(\mathrm{~d}, J=14.6 \mathrm{~Hz}, 2 \mathrm{H}), 3.16(\mathrm{~s}$, $3 \mathrm{H}) .{ }^{13} \mathrm{C}$ NMR $\left(100 \mathrm{MHz}\right.$, DMSO- $\left.d_{6}\right) \delta 156.5,140.0,139.0,137.3,129.4,129.0,128.6,128.1$, 128.0, 126.4, 112.7, 73.9, 62.7, 57.3, 52.9, 46.5. HRMS (ESI) calculated for $\mathrm{C}_{27} \mathrm{H}_{29} \mathrm{~N}_{2} \mathrm{O}_{3}[\mathrm{M}+\mathrm{H}]^{+}$: 429.2173, found: 429.2169 .

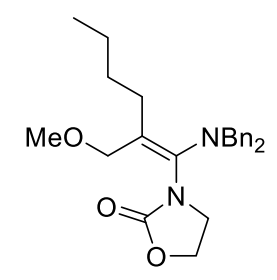

(Z)-3-(1-(dibenzylamino)-2-(methoxymethyl)hex-1-en-1-yl)oxazolidin-2-one (3at):

Obtained as colorless oil after column chromatography (EtOAc:Petroleum ether $=1: 10$ to 1:5), $30.6 \mathrm{mg}, 75 \%$ yield. ${ }^{1} \mathrm{H}$ NMR (400 MHz, DMSO- $\left.d_{6}\right) \delta$ 7.39-7.32 (m, 5H), 7.32-7.28 (m, 5H), 4.35 (t, $J=7.6 \mathrm{~Hz} 2 \mathrm{H}), 3.95-3.65(\mathrm{~m}, 6 \mathrm{H}), 3.56(\mathrm{t}, J=7.6 \mathrm{~Hz}, 2 \mathrm{H}), 3.09(\mathrm{~s}, 3 \mathrm{H}), 2.04-1.88(\mathrm{~m}, 1 \mathrm{H})$, $1.82-1.60(\mathrm{~m}, 1 \mathrm{H}), 1.40-1.24(\mathrm{~m}, 1 \mathrm{H}), 1.10-0.94(\mathrm{~m}, 3 \mathrm{H}), 0.76(\mathrm{t}, J=7.6 \mathrm{~Hz}, 3 \mathrm{H}) .{ }^{13} \mathrm{C}$ NMR $(100$ MHz, DMSO-d 6 ) $\delta 156.6,138.6,136.5,129.0,128.8,127.6,118.8,70.4,62.4,57.4,54.0,45.7$, 29.9, 28.6, 23.0, 14.4. HRMS (ESI) calculated for $\mathrm{C}_{25} \mathrm{H}_{33} \mathrm{~N}_{2} \mathrm{O}_{3}[\mathrm{M}+\mathrm{H}]^{+}: 409.2486$, found: 409.2488 .

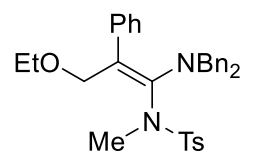

(Z)-N-(1-(dibenzylamino)-3-ethoxy-2-phenylprop-1-en-1-yl)-N,4-dimethylbenzenesulfonamide (3ba):

Obtained as colorless oil after column chromatography (EtOAc:Petroleum ether $=1: 50$ to 1:20), $48.0 \mathrm{mg}, 89 \%$ yield. ${ }^{1} \mathrm{H}$ NMR (400 MHz, DMSO- $\left.d_{6}\right) \delta 7.81(\mathrm{~d}, J=8.2 \mathrm{~Hz}, 2 \mathrm{H}), 7.43(\mathrm{~d}, J=8.2$ $\mathrm{Hz}, 2 \mathrm{H}), 7.39-7.29(\mathrm{~m}, 6 \mathrm{H}), 7.17(\mathrm{~d}, J=6.8 \mathrm{~Hz}, 4 \mathrm{H}), 7.11-7.03(\mathrm{~m}, 3 \mathrm{H}), 6.88-6.83(\mathrm{~m}, 2 \mathrm{H}), 3.79$ (s, 2H), $3.72(\mathrm{~d}, J=14.6 \mathrm{~Hz}, 2 \mathrm{H}), 3.42(\mathrm{~d}, J=14.6 \mathrm{~Hz}, 2 \mathrm{H}), 3.13-3.05(\mathrm{~m}, 2 \mathrm{H}), 3.00(\mathrm{~s}, 3 \mathrm{H}), 2.39$ (s, 3H), 0.91 (t, $J=7.0 \mathrm{~Hz}, 3 \mathrm{H}) .{ }^{13} \mathrm{C}$ NMR $\left(75 \mathrm{MHz}, \mathrm{DMSO}-d_{6}\right) \delta 144.7,144.3,140.2,137.3$, 136.0, 130.3, 129.6, 128.84, 128.78, 128.2, 127.9, 127.7, 126.4, 115.3, 71.5, 65.2, 53.4, 38.9, 21.5, 15.3. HRMS (ESI) calculated for $\mathrm{C}_{33} \mathrm{H}_{37} \mathrm{~N}_{2} \mathrm{O}_{3} \mathrm{~S}[\mathrm{M}+\mathrm{H}]^{+}: 541.2519$, found: 541.2516 .

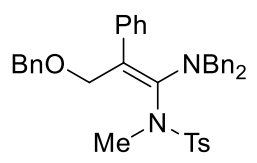

(Z)-N-(3-(benzyloxy)-1-(dibenzylamino)-2-phenylprop-1-en-1-yl)-N,4-dimethylbenzenesulfonam ide (3ca):

Obtained as colorless oil after column chromatography (EtOAc:Petroleum ether $=1: 50$ to 1:20), $47.0 \mathrm{mg}, 78 \%$ yield. ${ }^{1} \mathrm{H}$ NMR $\left(400 \mathrm{MHz}, \mathrm{DMSO}-d_{6}\right) \delta 7.74(\mathrm{~d}, J=8.2 \mathrm{~Hz}, 2 \mathrm{H}), 7.42-7.23(\mathrm{~m}$, $11 \mathrm{H}), 7.17(\mathrm{~d}, J=7.0 \mathrm{~Hz}, 4 \mathrm{H}), 7.13-7.06(\mathrm{~m}, 3 \mathrm{H}), 7.05-6.98(\mathrm{~m}, 2 \mathrm{H}), 6.88(\mathrm{~d}, J=6.1 \mathrm{~Hz}, 2 \mathrm{H})$, $4.13(\mathrm{q}, J=12.1 \mathrm{~Hz}, 2 \mathrm{H}), 3.85-3.69(\mathrm{~m}, 4 \mathrm{H}), 3.46$ (d, $J=14.5 \mathrm{~Hz}, 2 \mathrm{H}), 2.92(\mathrm{~s}, 3 \mathrm{H}), 2.40$ (s, 3H). 
${ }^{13} \mathrm{C}$ NMR $\left(75 \mathrm{MHz}, \mathrm{DMSO}-d_{6}\right) \delta 144.8,144.3,140.1,138.6,137.3,135.8,130.3,129.5,128.85$, 128.82, 128.6, 128.3, 128.0, 127.9, 127.8, 127.6, 126.5, 115.0, 72.0, 71.3, 53.5, 38.8, 21.5. HRMS (ESI) calculated for $\mathrm{C}_{38} \mathrm{H}_{39} \mathrm{~N}_{2} \mathrm{O}_{3} \mathrm{~S}[\mathrm{M}+\mathrm{H}]^{+}:$603.2676, found: 603.2682 .

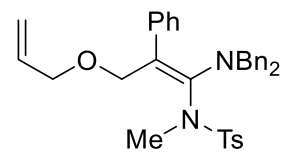

(Z)-N-(3-(allyloxy)-1-(dibenzylamino)-2-phenylprop-1-en-1-yl)-N,4-dimethylbenzenesulfonamid $e(3 d a)$ :

Obtained as colorless oil after column chromatography (EtOAc:Petroleum ether $=1: 50$ to 1:20), $45.3 \mathrm{mg}, 82 \%$ yield. ${ }^{1} \mathrm{H}$ NMR (400 MHz, DMSO- $\left.d_{6}\right) \delta 7.80(\mathrm{~d}, J=8.2 \mathrm{~Hz}, 2 \mathrm{H}), 7.43(\mathrm{~d}, J=8.2$ $\mathrm{Hz}, 2 \mathrm{H}), 7.39-7.28(\mathrm{~m}, 6 \mathrm{H}), 7.16(\mathrm{~d}, J=7.0 \mathrm{~Hz}, 4 \mathrm{H}), 7.12-7.05(\mathrm{~m}, 3 \mathrm{H}), 6.89-6.83(\mathrm{~m}, 2 \mathrm{H})$, $5.74-5.56(\mathrm{~m}, 1 \mathrm{H}), 5.01(\mathrm{~d}, J=13.4 \mathrm{~Hz}, 2 \mathrm{H}), 3.74(\mathrm{~d}, J=14.6 \mathrm{~Hz}, 4 \mathrm{H}), 3.66-3.54(\mathrm{~m}, 2 \mathrm{H}), 3.44(\mathrm{~d}$, $J=14.6 \mathrm{~Hz}, 2 \mathrm{H}), 2.98(\mathrm{~s}, 3 \mathrm{H}), 2.40(\mathrm{~s}, 3 \mathrm{H}) .{ }^{13} \mathrm{C}$ NMR $\left(75 \mathrm{MHz}, \mathrm{DMSO}-d_{6}\right) \delta 145.0,144.3,140.2$, 137.3, 135.9, 135.4, 130.3, 129.5, 128.9, 128.3, 127.9, 127.7, 126.5, 117.1, 115.1, 71.2, 70.9, 53.5, 39.0, 21.5. HRMS (ESI) calculated for $\mathrm{C}_{34} \mathrm{H}_{37} \mathrm{~N}_{2} \mathrm{O}_{3} \mathrm{~S}[\mathrm{M}+\mathrm{H}]^{+}$: 553.2519, found: 553.2517 .

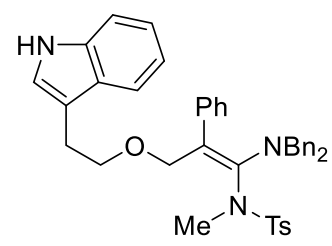

(Z)-N-(3-(2-(1H-indol-3-yl)ethoxy)-1-(dibenzylamino)-2-phenylprop-1-en-1-yl)-N,4-dimethylbe nzenesulfonamide (3ea):

Obtained as colorless oil after column chromatography (EtOAc:Petroleum ether $=1: 20$ to 1:10), $60.4 \mathrm{mg}, 92 \%$ yield. ${ }^{1} \mathrm{H}$ NMR (400 MHz, DMSO- $\left.d_{6}\right) \delta 7.79(\mathrm{~d}, J=8.1 \mathrm{~Hz}, 2 \mathrm{H}), 7.48(\mathrm{~d}, J=7.8$ $\mathrm{Hz}, 1 \mathrm{H}), 7.42-7.32(\mathrm{~m}, 6 \mathrm{H}), 7.30(\mathrm{~d}, J=8.1 \mathrm{~Hz}, 2 \mathrm{H}), 7.24-7.18(\mathrm{~m}, 5 \mathrm{H}), 7.07-6.94(\mathrm{~m}, 3 \mathrm{H}), 6.90$ (t, $J=7.6 \mathrm{~Hz}, 2 \mathrm{H}), 6.85(\mathrm{~s}, 1 \mathrm{H}), 6.54(\mathrm{~d}, J=7.6 \mathrm{~Hz}, 2 \mathrm{H}), 4.83-4.73(\mathrm{~m}, 2 \mathrm{H}), 4.60(\mathrm{t}, J=5.3 \mathrm{~Hz}$, 1H), $3.69(\mathrm{~d}, J=14.6 \mathrm{~Hz}, 2 \mathrm{H}), 3.58-3.45(\mathrm{~m}, 4 \mathrm{H}), 3.06(\mathrm{~s}, 3 \mathrm{H}), 2.75(\mathrm{t}, J=7.4 \mathrm{~Hz}, 2 \mathrm{H}), 2.33$ (s, $3 \mathrm{H}) .{ }^{13} \mathrm{C}$ NMR $\left(100 \mathrm{MHz}\right.$, DMSO- $\left.d_{6}\right) \delta 144.6,144.5,138.9,137.1,136.8,135.8,130.3,129.6$, 128.9, 128.8, 128.3, 128.2, 128.1, 127.8, 126.9, 125.7, 121.4, 119.1, 118.9, 113.4, 111.7, 110.1, 62.2, 53.4, 48.6, 38.0, 29.2, 21.4. HRMS (ESI) calculated for $\mathrm{C}_{41} \mathrm{H}_{42} \mathrm{~N}_{3} \mathrm{O}_{3} \mathrm{~S}[\mathrm{M}+\mathrm{H}]^{+}: 656.2941$, found: 656.2938 .

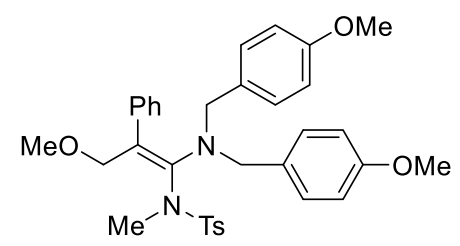

(Z)-N-(1-(bis(4-methoxybenzyl)amino)-3-methoxy-2-phenylprop-1-en-1-yl)-N,4-dimethylbenzen esulfonamide ( $3 f a)$ :

Obtained as colorless oil after column chromatography (EtOAc:Petroleum ether $=1: 20$ to 1:10), $42.2 \mathrm{mg}, 72 \%$ yield. ${ }^{1} \mathrm{H}$ NMR (400 MHz, DMSO- $\left.d_{6}\right) \delta 7.81(\mathrm{~d}, J=8.2 \mathrm{~Hz}, 2 \mathrm{H}), 7.43(\mathrm{~d}, J=8.2$ $\mathrm{Hz}, 2 \mathrm{H}), 7.13-7.03(\mathrm{~m}, 7 \mathrm{H}), 6.93(\mathrm{~d}, J=8.6 \mathrm{~Hz}, 4 \mathrm{H}), 6.83-6.78(\mathrm{~m}, 2 \mathrm{H}), 3.85(\mathrm{~d}, J=10.9 \mathrm{~Hz}, 1 \mathrm{H})$, 
3.80-3.74 (m, 7H), $3.61(\mathrm{~d}, J=14.5 \mathrm{~Hz}, 2 \mathrm{H}), 3.33(\mathrm{~d}, J=14.5 \mathrm{~Hz}, 2 \mathrm{H}), 3.06(\mathrm{~s}, 3 \mathrm{H}), 2.97(\mathrm{~s}, 3 \mathrm{H})$, $2.39(\mathrm{~s}, 3 \mathrm{H}) .{ }^{13} \mathrm{C}$ NMR $\left(100 \mathrm{MHz}, \mathrm{DMSO}-d_{6}\right) \delta 159.1,144.9,144.3,140.2,136.1,130.8,130.3$, 129.1, 128.7, 128.2, 127.7, 126.3, 114.19, 114.17, 73.8, 57.8, 55.6, 52.4, 39.0, 21.5. HRMS (ESI) calculated for $\mathrm{C}_{34} \mathrm{H}_{39} \mathrm{~N}_{2} \mathrm{O}_{5} \mathrm{~S}[\mathrm{M}+\mathrm{H}]^{+}:$587.2574, found: 587.2578 .

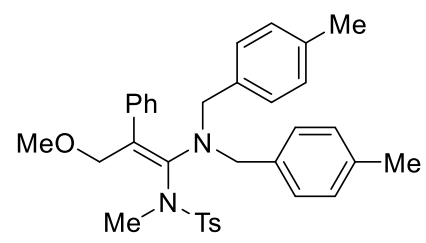

(Z)-N-(1-(bis(4-methylbenzyl)amino)-3-methoxy-2-phenylprop-1-en-1-yl)-N,4-dimethylbenzenes ulfonamide (3ga):

Obtained as colorless oil after column chromatography (EtOAc:Petroleum ether $=1: 50$ to 1:20), $48.8 \mathrm{mg}, 88 \%$ yield. ${ }^{1} \mathrm{H}$ NMR (400 MHz, DMSO- $\left.d_{6}\right) \delta 7.80(\mathrm{~d}, J=8.2 \mathrm{~Hz}, 2 \mathrm{H}), 7.43(\mathrm{~d}, J=8.2$ $\mathrm{Hz}, 2 \mathrm{H}), 7.17(\mathrm{~d}, J=7.8 \mathrm{~Hz}, 4 \mathrm{H}), 7.11-7.02(\mathrm{~m}, 7 \mathrm{H}), 6.80(\mathrm{~d}, J=6.7 \mathrm{~Hz}, 2 \mathrm{H}), 3.79(\mathrm{dd}, J=26.6$, $10.9 \mathrm{~Hz}, 2 \mathrm{H}), 3.63(\mathrm{~d}, J=14.5 \mathrm{~Hz}, 2 \mathrm{H}), 3.36-3.31(\mathrm{~m}, 2 \mathrm{H}), 3.03(\mathrm{~s}, 3 \mathrm{H}), 2.95(\mathrm{~s}, 3 \mathrm{H}), 2.39(\mathrm{~s}, 3 \mathrm{H})$ $2.31(\mathrm{~s}, 6 \mathrm{H}) .{ }^{13} \mathrm{C}$ NMR $\left(75 \mathrm{MHz}, \mathrm{DMSO}-d_{6}\right) \delta 145.0,144.3,140.2,137.1,136.0,134.2,130.2$, 129.5, 129.4, 128.7, 128.2, 127.7, 126.3, 114.5, 73.8, 57.8, 52.9, 39.0, 21.5, 21.2. HRMS (ESI) calculated for $\mathrm{C}_{34} \mathrm{H}_{39} \mathrm{~N}_{2} \mathrm{O}_{3} \mathrm{~S}[\mathrm{M}+\mathrm{H}]^{+}$: 555.2676, found: 555.2673 .<smiles>COCC(=C(N(C)C)N(Cc1ccc(Cl)cc1)Cc1ccc(Cl)cc1)c1ccccc1</smiles>

(Z)-N-(1-(bis(4-chlorobenzyl)amino)-3-methoxy-2-phenylprop-1-en-1-yl)-N,4-dimethylbenzenes ulfonamide (3ha):

Obtained as colorless oil after column chromatography (EtOAc:Petroleum ether $=1: 50$ to 1:20), $53.0 \mathrm{mg}, 89 \%$ yield. ${ }^{1} \mathrm{H}$ NMR (400 MHz, DMSO- $\left.d_{6}\right) \delta 7.82(\mathrm{~d}, J=8.1 \mathrm{~Hz}, 2 \mathrm{H}), 7.46(\mathrm{~d}, J=8.1$ $\mathrm{Hz}, 2 \mathrm{H}), 7.39$ (d, $J=8.4 \mathrm{~Hz}, 4 \mathrm{H}), 7.17-7.10(\mathrm{~m}, 7 \mathrm{H}), 6.94-6.89(\mathrm{~m}, 2 \mathrm{H}), 3.80(\mathrm{~d}, J=14.8 \mathrm{~Hz}, 2 \mathrm{H})$, $3.69(\mathrm{~d}, J=11.0 \mathrm{~Hz}, 1 \mathrm{H}), 3.60(\mathrm{~d}, J=11.0 \mathrm{~Hz}, 1 \mathrm{H}), 3.47$ (d, $J=14.8 \mathrm{~Hz}, 2 \mathrm{H}), 2.94(\mathrm{~s}, 3 \mathrm{H}), 2.90$ (s, 3H), 2.41 (s, 3H). ${ }^{13} \mathrm{C}$ NMR (100 MHz, DMSO- $\left.d_{6}\right) \delta 144.7,144.4,140.0,136.4,135.7,132.5$, 131.2, 130.4, 128.9, 128.8, 128.4, 127.8, 126.7, 116.6, 73.5, 57.8, 53.2, 39.0, 21.5. HRMS (ESI) calculated for $\mathrm{C}_{32} \mathrm{H}_{33} \mathrm{Cl}_{2} \mathrm{~N}_{2} \mathrm{O}_{3} \mathrm{~S}[\mathrm{M}+\mathrm{H}]^{+}:$595.1583, found: 595.1586.<smiles>COCC(=C(N(C)C)N(Cc1ccccc1Cl)Cc1ccccc1Cl)P(C)C</smiles>

(Z)-N-(1-(bis(2-chlorobenzyl)amino)-3-methoxy-2-phenylprop-1-en-1-yl)-N,4-dimethylbenzenes ulfonamide (3ia):

Obtained as colorless oil after column chromatography (EtOAc:Petroleum ether $=1: 50$ to 1:20), $37.5 \mathrm{mg}, 63 \%$ yield. ${ }^{1} \mathrm{H}$ NMR (400 MHz, DMSO- $\left.d_{6}\right) \delta 7.77(\mathrm{~d}, J=8.2 \mathrm{~Hz}, 2 \mathrm{H}), 7.44(\mathrm{~d}, J=8.2$ 
$\mathrm{Hz}, 2 \mathrm{H}), 7.36-7.31(\mathrm{~m}, 2 \mathrm{H}), 7.29-7.19(\mathrm{~m}, 8 \mathrm{H}), 7.17-7.10(\mathrm{~m}, 3 \mathrm{H}), 4.23(\mathrm{~d}, J=16.0 \mathrm{~Hz}, 2 \mathrm{H}), 3.77$ $(\mathrm{d}, J=16.0 \mathrm{~Hz}, 2 \mathrm{H}), 3.51(\mathrm{q}, J=11.0 \mathrm{~Hz}, 2 \mathrm{H}), 2.89$ (s, 3H), $2.83(\mathrm{~s}, 3 \mathrm{H}), 2.41(\mathrm{~s}, 3 \mathrm{H}) .{ }^{13} \mathrm{C} \mathrm{NMR}$ (75 MHz, DMSO- $d_{6}$ ) $\delta 145.1,144.5,140.0,135.5,135.4,132.8,130.4,130.2,129.6,129.3,129.2$, 128.6, 127.8, 127.3, 126.9, 118.9, 73.4, 57.9, 52.6, 38.7, 21.5. HRMS (ESI) calculated for $\mathrm{C}_{32} \mathrm{H}_{33} \mathrm{Cl}_{2} \mathrm{~N}_{2} \mathrm{O}_{3} \mathrm{~S}[\mathrm{M}+\mathrm{H}]^{+}:$595.1583, found: 595.1580 .

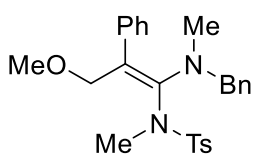

(Z)-N-(1-(benzyl(methyl)amino)-3-methoxy-2-phenylprop-1-en-1-yl)-N,4-dimethylbenzenesulfo namide (3ja):

Obtained as colorless oil after column chromatography (EtOAc:Petroleum ether $=1: 50$ to 1:20), $33.3 \mathrm{mg}, 74 \%$ yield. ${ }^{1} \mathrm{H}$ NMR (400 MHz, DMSO- $\left.d_{6}\right) \delta 7.74(\mathrm{~d}, J=8.1 \mathrm{~Hz}, 2 \mathrm{H}), 7.41(\mathrm{~d}, J=8.1$ $\mathrm{Hz}, 2 \mathrm{H}), 7.35-7.28(\mathrm{~m}, 3 \mathrm{H}), 7.17-7.07(\mathrm{~m}, 5 \mathrm{H}), 6.99-6.92(\mathrm{~m}, 2 \mathrm{H}), 3.90(\mathrm{q}, J=11.0 \mathrm{~Hz}, 2 \mathrm{H}), 3.71$ (d, $J=14.1 \mathrm{~Hz}, 1 \mathrm{H}), 3.32$ (d, $J=14.1 \mathrm{~Hz}, 1 \mathrm{H}), 3.16$ (s, 3H), 3.04 (s, 3H), 2.39 (s, 3H), 2.03 (s, $3 \mathrm{H}) .{ }^{13} \mathrm{C}$ NMR (100 MHz, DMSO- $\left.d_{6}\right) \delta 145.3,144.0,140.4,137.1,136.6,130.2,129.5,128.8$, $128.7,128.3,127.9,127.7,126.1,113.0,73.5,57.6,56.9,38.9,38.5,21.5$. HRMS (ESI) calculated for $\mathrm{C}_{26} \mathrm{H}_{31} \mathrm{~N}_{2} \mathrm{O}_{3} \mathrm{~S}[\mathrm{M}+\mathrm{H}]^{+}: 451.2050$, found: 451.2054 .

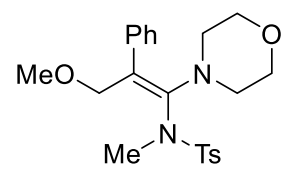

(Z)-N-(3-methoxy-1-morpholino-2-phenylprop-1-en-1-yl)-N,4-dimethylbenzenesulfonamide (3ka):

Obtained as colorless oil after column chromatography (EtOAc:Petroleum ether $=1: 20$ to 1:5), $28.3 \mathrm{mg}, 68 \%$ yield. ${ }^{1} \mathrm{H}$ NMR (400 MHz, DMSO- $\left.d_{6}\right) \delta 7.79(\mathrm{~d}, J=8.2 \mathrm{~Hz}, 2 \mathrm{H}), 7.47$ (d, $J=8.2$ $\mathrm{Hz}, 2 \mathrm{H}), 7.36-7.28(\mathrm{~m}, 2 \mathrm{H}), 7.22-7.15(\mathrm{~m}, 3 \mathrm{H}), 3.79(\mathrm{q}, J=11.0 \mathrm{~Hz}, 2 \mathrm{H}), 3.34-3.26(\mathrm{~m}, 2 \mathrm{H})$, 3.21-3.11 (m, 5H), $3.01(\mathrm{~s}, 3 \mathrm{H}), 2.50-2.45(\mathrm{~m}, 4 \mathrm{H}), 2.42(\mathrm{~s}, 3 \mathrm{H}) .{ }^{13} \mathrm{C}$ NMR (100 MHz, DMSO- $\left.d_{6}\right)$ $\delta 144.9,144.1,140.3,136.9,130.3,129.0,128.5,127.7,126.5,113.8,73.3,65.8,57.6,49.4,38.4$, 21.5. HRMS (ESI) calculated for $\mathrm{C}_{22} \mathrm{H}_{29} \mathrm{~N}_{2} \mathrm{O}_{4} \mathrm{~S}[\mathrm{M}+\mathrm{H}]^{+}: 417.1843$, found: 417.1840 .

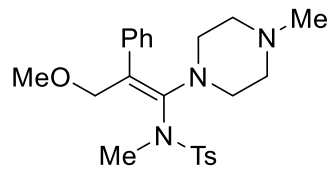

(Z)-N-(3-methoxy-1-(4-methylpiperazin-1-yl)-2-phenylprop-1-en-1-yl)-N,4-dimethylbenzenesulf onamide (3la):

Obtained as colorless oil after column chromatography (EtOAc:Petroleum ether $=1: 20$ to 1:5), $33.1 \mathrm{mg}, 77 \%$ yield. ${ }^{1} \mathrm{H}$ NMR (400 MHz, DMSO- $\left.d_{6}\right) \delta 7.78(\mathrm{~d}, J=8.2 \mathrm{~Hz}, 2 \mathrm{H}), 7.46$ (d, $J=8.2$ $\mathrm{Hz}, 2 \mathrm{H}), 7.33-7.27(\mathrm{~m}, 2 \mathrm{H}), 7.20-7.13(\mathrm{~m}, 3 \mathrm{H}), 3.87-3.77(\mathrm{~m}, 2 \mathrm{H}), 3.12(\mathrm{~s}, 3 \mathrm{H}), 3.03(\mathrm{~s}, 3 \mathrm{H})$, 2.49-2.43 (m, 4H), $2.42(\mathrm{~s}, 3 \mathrm{H}), 2.05-1.97(\mathrm{~m}, 5 \mathrm{H}), 1.92-1.83(\mathrm{~m}, 2 \mathrm{H}) .{ }^{13} \mathrm{C}$ NMR $(75 \mathrm{MHz}$, DMSO- $\left.d_{6}\right) \delta 144.8,144.0,140.5137 .0,130.2,128.9,128.4,127.7,126.3,113.3,73.5,57.4,54.2$, 
48.9, 46.2, 38.2, 21.5. HRMS (ESI) calculated for $\mathrm{C}_{23} \mathrm{H}_{32} \mathrm{~N}_{3} \mathrm{O}_{3} \mathrm{~S}[\mathrm{M}+\mathrm{H}]^{+}: 430.2159$, found: 430.2162 .

\section{Preparation of 3aa in $2 \mathrm{mmol}$ scale}<smiles>COCN(Cc1ccccc1)Cc1ccccc1</smiles>

1 a

$482.3 \mathrm{mg}, 2 \mathrm{mmol}$

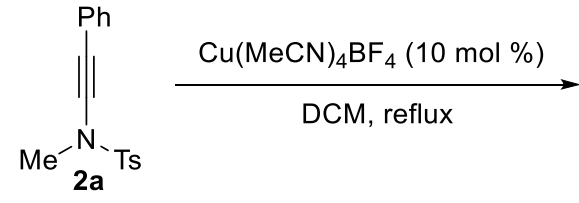

$684.8 \mathrm{mg}, 2.4 \mathrm{mmol}$

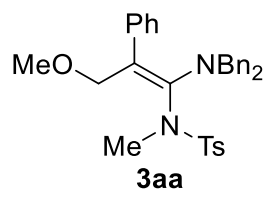

$863.8 \mathrm{mg}, 82 \%$

To a tube was added $\mathrm{Cu}(\mathrm{MeCN})_{4} \mathrm{BF}_{4}(63 \mathrm{mg}, 0.2 \mathrm{mmol}), \mathbf{1 a}(482.3 \mathrm{mg}, 2 \mathrm{mmol}), 2 \mathrm{a}(684.8 \mathrm{mg}$, $2.4 \mathrm{mmol})$ and $\mathrm{CH}_{2} \mathrm{Cl}_{2}(40 \mathrm{~mL})$ under an argon atmosphere. Then, the mixture was stirred at reflux for $6 \mathrm{~h}$. The reaction mixture was concentrated under vacuum, the crude residue was purified by column chromatography (basic aluminium oxide, EtOAc: Petroleum ether $=1: 50$ to 1:20) to give 3aa (863.8 $\mathrm{mg}, 82 \%)$.

\section{Control experiments for Scheme 4}

\section{(1) Scheme 4-a}
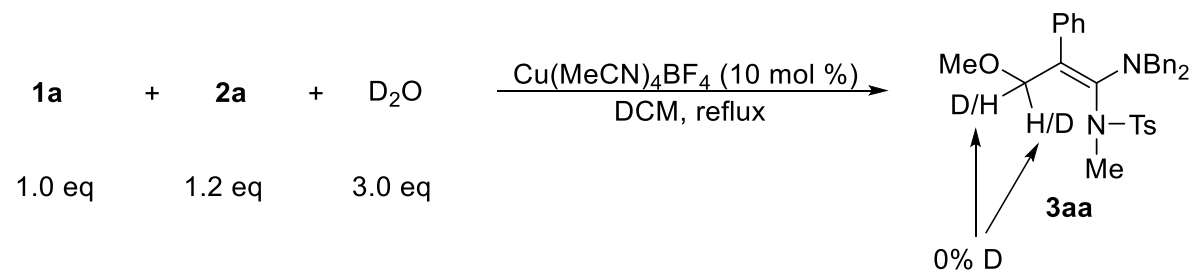

To a tube was added $\mathrm{Cu}(\mathrm{MeCN})_{4} \mathrm{BF}_{4}(3.2 \mathrm{mg}, 0.01 \mathrm{mmol}), \mathbf{1 a}(24.1 \mathrm{mg}, 0.1 \mathrm{mmol}), \mathbf{2 a}(34.2 \mathrm{mg}$, $0.12 \mathrm{mmol}), \mathrm{D}_{2} \mathrm{O}(6.0 \mathrm{mg} 0.3 \mathrm{mmol})$ and $\mathrm{CH}_{2} \mathrm{Cl}_{2}(2 \mathrm{~mL})$ under an argon atmosphere. Then, the mixture was stirred at reflux for $6 \mathrm{~h}$. The reaction mixture was concentrated under vacuum, the crude residue was purified by column chromatography (basic aluminium oxide, EtOAc: Petroleum ether $=1: 50$ to $1: 20)$ to give $\mathbf{3 a a}(42.1 \mathrm{mg}, 80 \%)$.

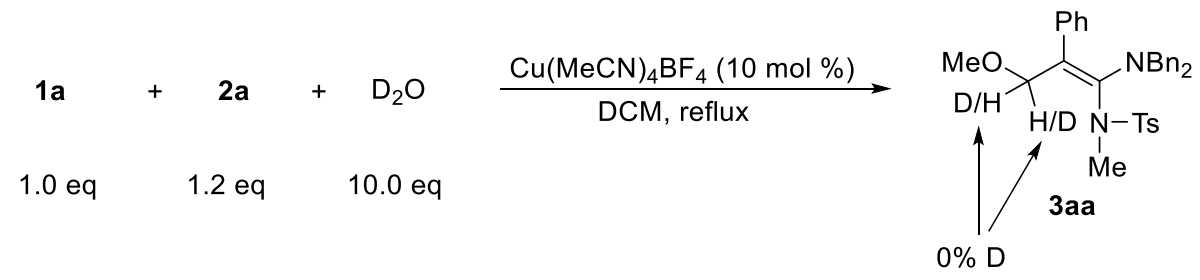

To a tube was added $\mathrm{Cu}(\mathrm{MeCN})_{4} \mathrm{BF}_{4}(3.2 \mathrm{mg}, 0.01 \mathrm{mmol}), \mathbf{1 a}(24.1 \mathrm{mg}, 0.1 \mathrm{mmol}), \mathbf{2 a}(34.2 \mathrm{mg}$, $0.12 \mathrm{mmol}), \mathrm{D}_{2} \mathrm{O}(20 \mathrm{mg} 1.0 \mathrm{mmol})$ and $\mathrm{CH}_{2} \mathrm{Cl}_{2}(2 \mathrm{~mL})$ under an argon atmosphere. Then, the mixture was stirred at reflux for $6 \mathrm{~h}$. The reaction mixture was concentrated under vacuum, the crude residue was purified by column chromatography (basic aluminium oxide, EtOAc: Petroleum ether $=1: 50$ to $1: 20)$ to give $\mathbf{3 a a}(40 \mathrm{mg}, 74 \%)$.

\section{(2) Scheme 4-b}




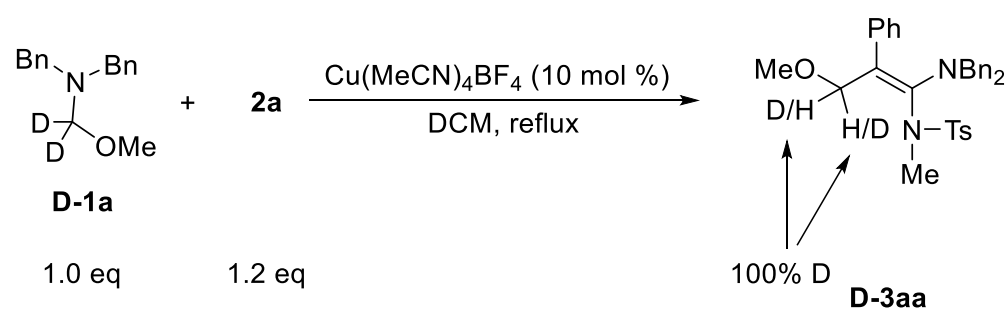

To a tube was added $\mathrm{Cu}(\mathrm{MeCN})_{4} \mathrm{BF}_{4}(3.2 \mathrm{mg}, 0.01 \mathrm{mmol}), \mathbf{D}-1 \mathbf{a}(24.3 \mathrm{mg}, 0.1 \mathrm{mmol}), \mathbf{2 a}(34.2 \mathrm{mg}$, $0.12 \mathrm{mmol})$ and $\mathrm{CH}_{2} \mathrm{Cl}_{2}(2 \mathrm{~mL})$ under an argon atmosphere. Then, the mixture was stirred at reflux for $6 \mathrm{~h}$. The reaction mixture was concentrated under vacuum, the crude product was purified by column chromatography (basic aluminium oxide, EtOAc: Petroleum ether $=1: 50$ to $1: 20)$ to give D-3aa (43.3 mg, 82\%). ${ }^{1} \mathrm{H}$ NMR (400 MHz, DMSO- $\left.d_{6}\right) \delta 7.81(\mathrm{~d}, J=8.1 \mathrm{~Hz}, 2 \mathrm{H})$, $7.43(\mathrm{~d}, J=8.1 \mathrm{~Hz}, 2 \mathrm{H}), 7.39-7.29(\mathrm{~m}, 6 \mathrm{H}), 7.17(\mathrm{~d}, J=6.8 \mathrm{~Hz}, 4 \mathrm{H}), 7.11-7.03(\mathrm{~m}, 3 \mathrm{H}), 6.86-6.80$ $(\mathrm{m}, 2 \mathrm{H}), 3.71(\mathrm{~d}, J=14.5 \mathrm{~Hz}, 2 \mathrm{H}), 3.43-3.40(\mathrm{~m}, 2 \mathrm{H}), 3.02(\mathrm{~s}, 3 \mathrm{H}), 2.94(\mathrm{~s}, 3 \mathrm{H}), 2.39(\mathrm{~s}, 3 \mathrm{H}) .{ }^{13} \mathrm{C}$ NMR (100 MHz, DMSO- $\left.d_{6}\right) \delta 144.9,144.3,140.1,137.2,135.9,130.3,129.6,128.9,128.7,128.2$, 127.9, 127.7, 126.4, 114.8, 57.7, 53.4, 39.0, 21.5. HRMS (ESI) calculated for $\mathrm{C}_{32} \mathrm{H}_{33} \mathrm{D}_{2} \mathrm{~N}_{2} \mathrm{O}_{3} \mathrm{~S}$ $[\mathrm{M}+\mathrm{H}]^{+}:$529.2488, found: 529.2485 .

\section{(3) Scheme 4-c}

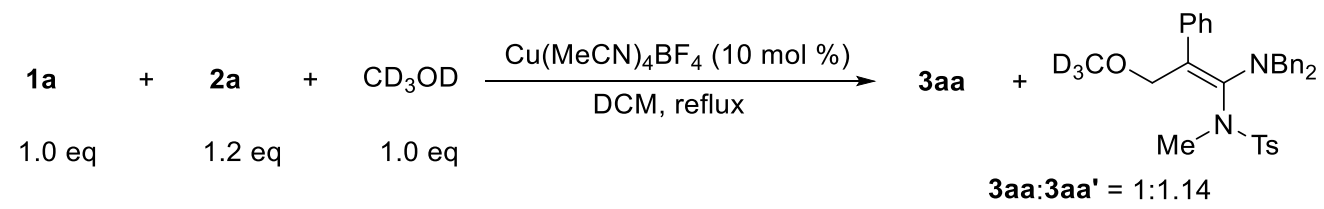

To a tube was added $\mathrm{Cu}(\mathrm{MeCN})_{4} \mathrm{BF}_{4}(3.2 \mathrm{mg}, 0.01 \mathrm{mmol}), \mathbf{1 a}(24.1 \mathrm{mg}, 0.1 \mathrm{mmol}), \mathbf{2 a}(34.2 \mathrm{mg}$, $0.12 \mathrm{mmol}), \mathrm{CD}_{3} \mathrm{OD}(3.6 \mathrm{mg}, 0.1 \mathrm{mmol})$ and $\mathrm{CH}_{2} \mathrm{Cl}_{2}(2 \mathrm{~mL})$ under an argon atmosphere. Then, the mixture was stirred at reflux for $6 \mathrm{~h}$. The reaction mixture was concentrated under vacuum, the crude product was purified by column chromatography (basic aluminium oxide, EtOAc: Petroleum ether $=1: 50$ to 1:20) to give a mixture of 3aa and 3aa' $(42.8 \mathrm{mg}$, ratio of 3aa:3aa' $=$ 1:1.14). ${ }^{1} \mathrm{H}$ NMR (400 MHz, DMSO- $\left.d_{6}\right) \delta 7.81(\mathrm{~d}, J=8.3 \mathrm{~Hz}, 2 \mathrm{H}), 7.43(\mathrm{~d}, J=8.1 \mathrm{~Hz}, 2 \mathrm{H})$, 7.39-7.28 (m, 6H), $7.17(\mathrm{~d}, J=6.8 \mathrm{~Hz}, 4 \mathrm{H}), 7.11-7.03(\mathrm{~m}, 3 \mathrm{H}), 6.85-6.79(\mathrm{~m}, 2 \mathrm{H}), 3.81(\mathrm{~d}, J=$ $10.9 \mathrm{~Hz}, 1 \mathrm{H}), 3.76-3.67$ (m, 3H), 3.43-3.39 (m, 2H), 3.02 (s, 3H), 2.94 (s, 1.4H), 2.39 (s, 3H).

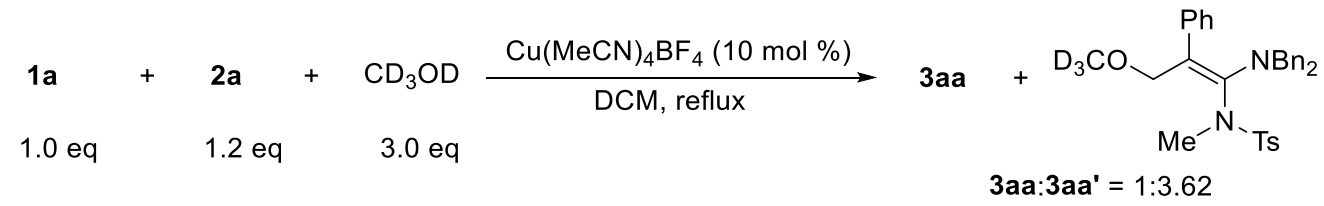

To a tube was added $\mathrm{Cu}(\mathrm{MeCN})_{4} \mathrm{BF}_{4}(3.2 \mathrm{mg}, 0.01 \mathrm{mmol}), \mathbf{1 a}(24.1 \mathrm{mg}, 0.1 \mathrm{mmol}), \mathbf{2 a}(34.2 \mathrm{mg}$, $0.12 \mathrm{mmol}), \mathrm{CD}_{3} \mathrm{OD}(10.8 \mathrm{mg}, 0.6 \mathrm{mmol})$ and $\mathrm{CH}_{2} \mathrm{Cl}_{2}(2 \mathrm{~mL})$ under an argon atmosphere. Then, the mixture was stirred at reflux for $6 \mathrm{~h}$. The reaction mixture was concentrated under vacuum, the crude product was purified by column chromatography (basic aluminium oxide, EtOAc: Petroleum ether $=1: 50$ to 1:20) to give a mixture of 3aa and 3aa' $(42.2 \mathrm{mg}$, ratio of 3aa:3aa' $=$ 1:3.62). ${ }^{1} \mathrm{H}$ NMR $\left(400 \mathrm{MHz}, \mathrm{DMSO}-d_{6}\right) \delta 7.81(\mathrm{~d}, J=8.2 \mathrm{~Hz}, 2 \mathrm{H}), 7.43(\mathrm{~d}, J=8.2 \mathrm{~Hz}, 2 \mathrm{H})$, 7.40-7.29 (m, 6H), $7.17(\mathrm{~d}, J=6.8 \mathrm{~Hz}, 4 \mathrm{H}), 7.12-7.03(\mathrm{~m}, 3 \mathrm{H}), 6.86-6.78(\mathrm{~m}, 2 \mathrm{H}), 3.80(\mathrm{~d}, J=$ $10.9 \mathrm{~Hz}, 1 \mathrm{H}), 3.76-3.66(\mathrm{~m}, 3 \mathrm{H}), 3.44-3.39(\mathrm{~m}, 2 \mathrm{H}), 3.02(\mathrm{~s}, 3 \mathrm{H}), 2.94(\mathrm{~s}, 0.65 \mathrm{H}), 2.40(\mathrm{~s}, 3 \mathrm{H})$ 


\section{(4) Scheme 4-d}

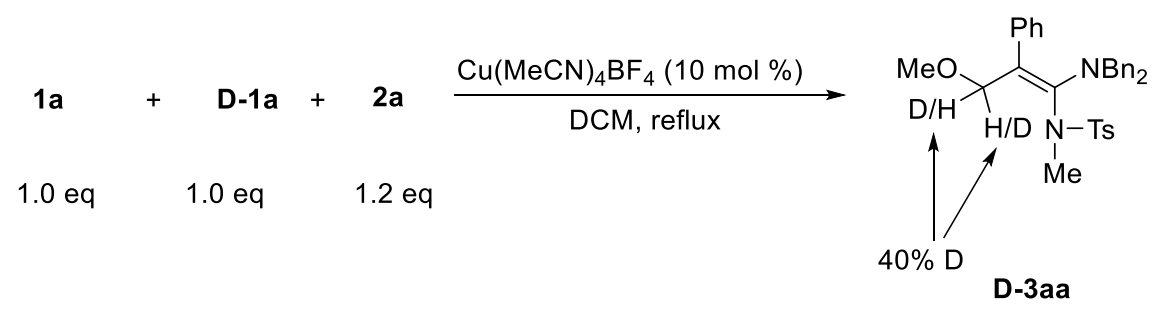

To a tube was added $\mathrm{Cu}(\mathrm{MeCN}){ }_{4} \mathrm{BF}_{4}(3.2 \mathrm{mg}, 0.01 \mathrm{mmol}), \mathbf{1 a}(24.1 \mathrm{mg}, 0.1 \mathrm{mmol}), \mathbf{D}-1 \mathbf{a}(24.3$ $\mathrm{mg}, 0.1 \mathrm{mmol}) 2 \mathrm{a}(34.2 \mathrm{mg}, 0.12 \mathrm{mmol})$ and $\mathrm{CH}_{2} \mathrm{Cl}_{2}(2 \mathrm{~mL})$ under an argon atmosphere. Then, the mixture was stirred at reflux for $6 \mathrm{~h}$. The reaction mixture was concentrated under vacuum, the crude product was purified by column chromatography (basic aluminium oxide, EtOAc: Petroleum ether $=1: 50$ to 1:20) to give a mixture of 3aa and D-3aa (43.6 mg, 40\% D). ${ }^{1} \mathrm{H}$ NMR $\left(300 \mathrm{MHz}, \mathrm{DMSO}-d_{6}\right) \delta 7.81(\mathrm{~d}, J=8.3 \mathrm{~Hz}, 2 \mathrm{H}), 7.43(\mathrm{~d}, J=8.3 \mathrm{~Hz}, 2 \mathrm{H}), 7.39-7.30(\mathrm{~m}, 6 \mathrm{H})$, 7.20-7.13 (m, 4H), 7.11-7.03 (m, 3H), 6.85-6.78 (m, 2H), $3.80(\mathrm{~d}, J=10.9 \mathrm{~Hz}, 0.6 \mathrm{H}), 3.75-3.66$ (m, 3H), 3.43-3.40 (m, 2H), $3.02(\mathrm{~s}, 3 \mathrm{H}), 2.94(\mathrm{~s}, 3 \mathrm{H}), 2.40$ (s, 3H).

\section{(5) Scheme 4-e}

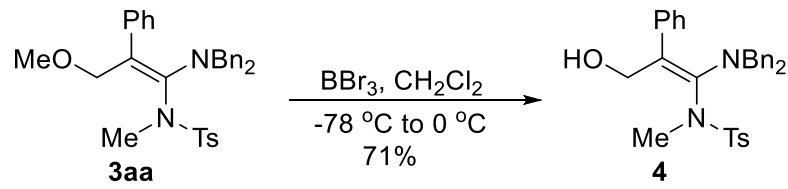

Under an argon atmosphere, to a solution of 3aa $(52.7 \mathrm{mg}, 0.1 \mathrm{mmol})$ in $\mathrm{CH}_{2} \mathrm{Cl}_{2}(5 \mathrm{~mL})$ was added a solution of $\mathrm{BBr}_{3}$ in $\mathrm{CH}_{2} \mathrm{Cl}_{2}(0.5 \mathrm{~mL}, 0.5 \mathrm{mmol}, 1 \mathrm{~mol} / \mathrm{L})$ dropwisely at $-78^{\circ} \mathrm{C}$. After stirring at $-78^{\circ} \mathrm{C}$ for $1 \mathrm{~h}$, the reaction mixture was slowly warmed to $0^{\circ} \mathrm{C}$. The reaction was stirred at $0{ }^{\circ} \mathrm{C}$ for $3 \mathrm{~h}$. The reaction mixture was quenched with $\mathrm{H}_{2} \mathrm{O}(10 \mathrm{~mL})$ and extracted with $\mathrm{CH}_{2} \mathrm{Cl}_{2}$ ( $5 \mathrm{ml}$ x 3). The combined organic layers were dried over $\mathrm{Na}_{2} \mathrm{SO}_{4}$, evaporated to provide crude product, which was purified by column chromatography. 4 was obtained as colorless oil after column chromatography (basic aluminium oxide, EtOAc:Petroleum ether $=1: 20$ to 1:3), $36.4 \mathrm{mg}$, $71 \%$ yield. ${ }^{1} \mathrm{H}$ NMR $\left(400 \mathrm{MHz}, \mathrm{DMSO}-d_{6}\right) \delta 7.81(\mathrm{~d}, J=8.0 \mathrm{~Hz}, 2 \mathrm{H}), 7.43(\mathrm{~d}, J=8.0 \mathrm{~Hz}, 2 \mathrm{H})$, 7.37-7.26 (m, 6H), 7.17-7.07 (m, 7H), 6.94-6.86 (m, 2H), $4.30(\mathrm{t}, J=4.9 \mathrm{~Hz}, 1 \mathrm{H}), 3.90-3.80(\mathrm{~m}$, 2H), $3.74(\mathrm{~d}, J=14.5 \mathrm{~Hz}, 2 \mathrm{H}), 3.44(\mathrm{~d}, J=14.5 \mathrm{~Hz}, 2 \mathrm{H}), 2.97(\mathrm{~s}, 3 \mathrm{H}), 2.40(\mathrm{~s}, 3 \mathrm{H}) .{ }^{13} \mathrm{C}$ NMR $\left(100 \mathrm{MHz}, \mathrm{DMSO}-d_{6}\right) \delta 144.2,143.7,140.3,137.7,136.0,130.3,129.5,129.2,128.8,128.2$, 127.82, 127.80, 126.3, 119.9, 62.5, 53.7, 39.2, 21.5. HRMS (ESI) calculated for $\mathrm{C}_{31} \mathrm{H}_{33} \mathrm{~N}_{2} \mathrm{O}_{3} \mathrm{~S}$ $[\mathrm{M}+\mathrm{H}]^{+}:$513.2206, found: 513.2203.

\section{(6) Scheme 4-f}

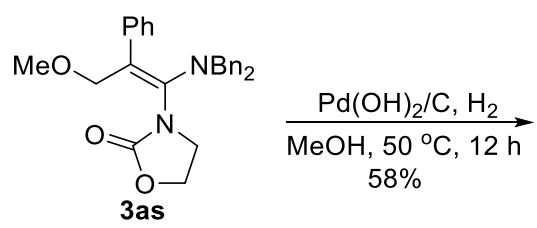<smiles></smiles>

To a $25 \mathrm{~mL}$ autoclave was added 3as $(21.4 \mathrm{mg}, 0.05 \mathrm{mmol}), 10 \% \mathrm{Pd}(\mathrm{OH})_{2} / \mathrm{C}(5 \mathrm{mg})$, and $\mathrm{MeOH}$ $(4 \mathrm{~mL})$. Then the mixture was stirred under $1.2 \mathrm{MPa}$ of $\mathrm{H}_{2}$ atmosphere at $50^{\circ} \mathrm{C}$ for $12 \mathrm{~h}$. The reaction mixture was cooled, filtered through a pad of celite, washed the cake with $\mathrm{MeOH}(3 \mathrm{~mL})$, 
and concentrated under vacuum to give crude product. 5 was obtained as a white solid after column chromatography (basic aluminium oxide, EtOAc:Petroleum ether $=1: 30$ to 1:6), $11.5 \mathrm{mg}$, 58\% yield, mp: $94-96{ }^{\circ} \mathrm{C} .{ }^{1} \mathrm{H}$ NMR $\left(400 \mathrm{MHz}, \mathrm{DMSO}-d_{6}\right) \delta$ 7.40-7.27 (m, 6H), $7.15(\mathrm{~d}, J=6.9$ $\mathrm{Hz}, 4 \mathrm{H}), 7.07$ (t, $J=7.5 \mathrm{~Hz}, 1 \mathrm{H}), 6.99(\mathrm{t}, J=7.5 \mathrm{~Hz}, 2 \mathrm{H}), 6.61(\mathrm{~d}, J=7.5 \mathrm{~Hz}, 2 \mathrm{H}), 4.45$ (t, $J=7.9$ $\mathrm{Hz}, 2 \mathrm{H}), 3.89-3.79(\mathrm{~m}, 1 \mathrm{H}), 3.76-3.69(\mathrm{~m}, 1 \mathrm{H}), 3.65(\mathrm{~d}, J=14.5 \mathrm{~Hz}, 2 \mathrm{H}), 3.40-3.34(\mathrm{~m}, 2 \mathrm{H}) 1.85$ (s, 3H). ${ }^{13} \mathrm{C}$ NMR (100 MHz, DMSO- $\left.d_{6}\right) \delta 155.5,141.0,137.2,134.9,129.0,128.4,127.8,127.7$, 127.3, 126.0, 113.6, 62.1, 52.7, 45.1, 20.1. HRMS (ESI) calculated for $\mathrm{C}_{26} \mathrm{H}_{27} \mathrm{~N}_{2} \mathrm{O}_{2}[\mathrm{M}+\mathrm{H}]^{+}$: 399.2067, found: 399.2064 .

\section{X-ray structure of 3ac and 3am}

The crystal structures have been deposited at the Cambridge Crystallographic Data Centre (CCDC 1956453, 3ac) and (CCDC 1956455, 3am). The data can be obtained free of charge via the internet at https://www.ccdc.cam.ac.uk/structures/.

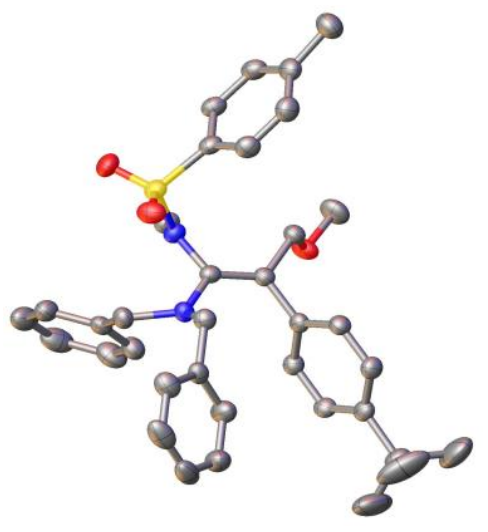

X-ray structure of 3ac

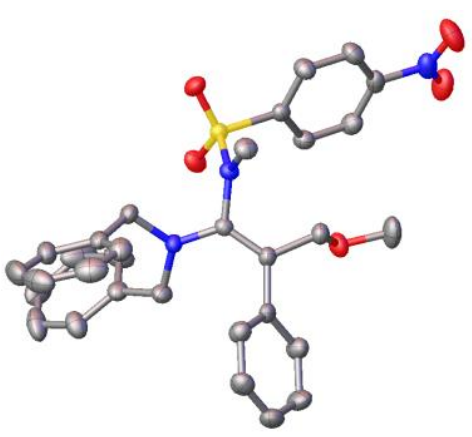

X-ray structure of 3am

\section{References}

1. (a) Chi, Y.; English, E. P.; Pomerantz, W. C.; Horne, W. S.; Joyce, L. A.; Alexander, L. R.; Fleming, W. S.; Hopkins, E. A.; Gellman, S. H. J. Am. Chem. Soc. 2007, 129, 6050. (b) Martinez-Cuezva, A.; Lopez-Leonardo, C.; Bautista, D.; Alajarin, M.; Berna, J. J. Am. Chem. Soc. 2016, 138, 8726.

2. (a) Tian, X.; Song, X.; Rudolph, M.; Wang, Q.; Song, X.; Rominger, F.; Hashmi, A. S. K. Org. Lett. 2019, 21, 1598. (b) Vanjari, R.; Dutta, S.; Gogoi, M. P.; Gandon, V.; Sahoo, A. K. Org. Lett. 2018, 20, 8077. 


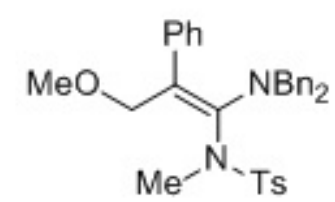

3aa, $400 \mathrm{MHz}, \mathrm{DMSO}-d_{6}$

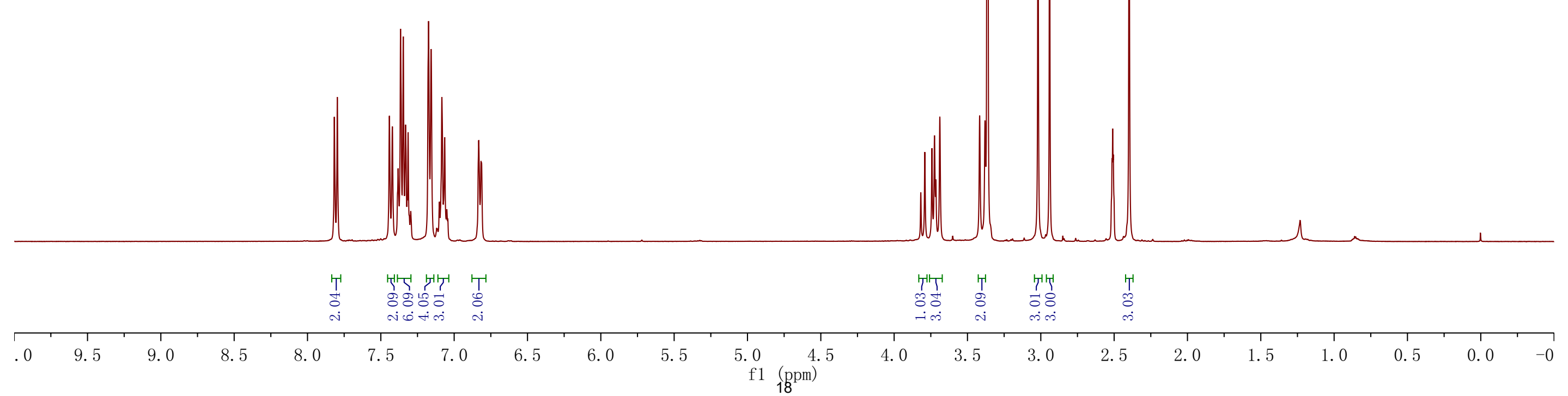




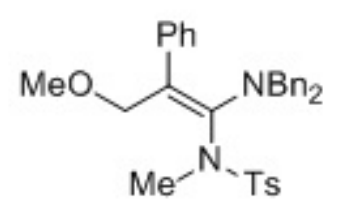

3aa, $100 \mathrm{MHz}, \mathrm{DMSO}-d_{6}$

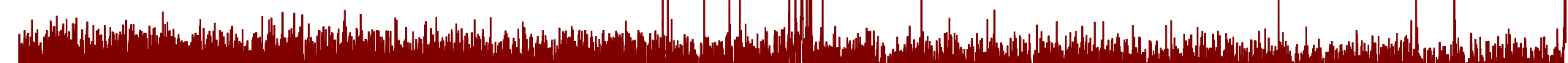

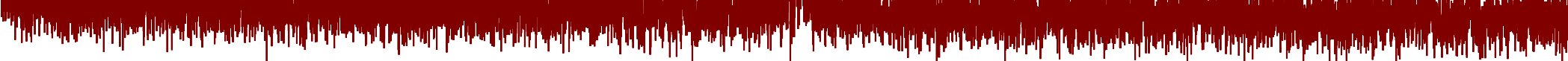

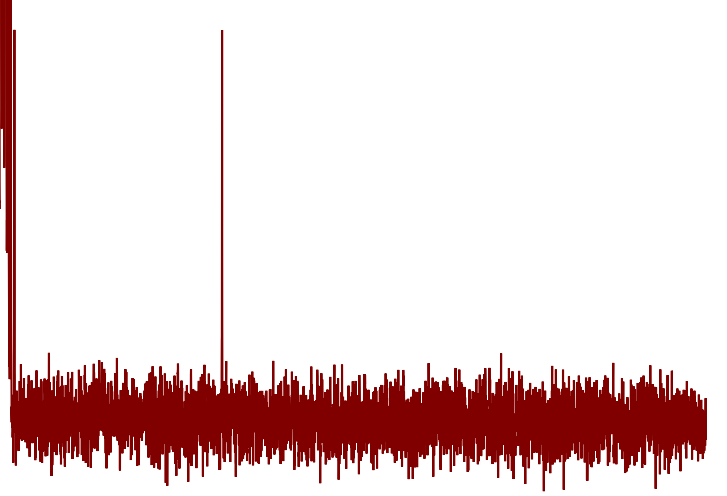

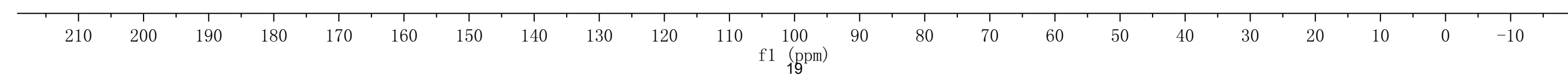




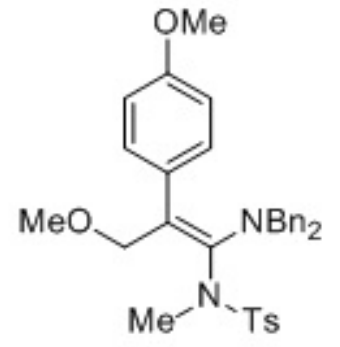

3ab, $400 \mathrm{MHz}, \mathrm{DMSO}-d_{6}$

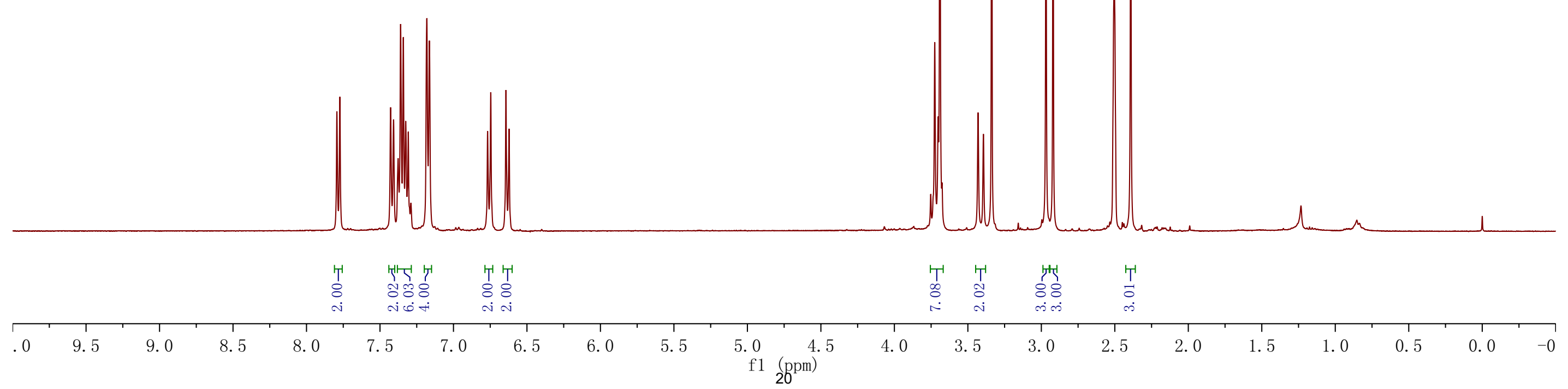




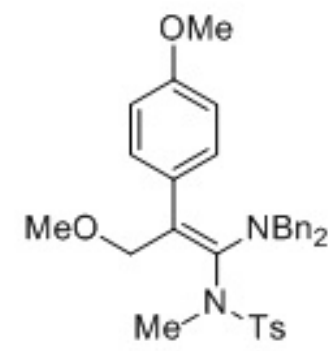

$3 \mathrm{ab}, 75 \mathrm{MHz}$, DMSO- $d_{6}$

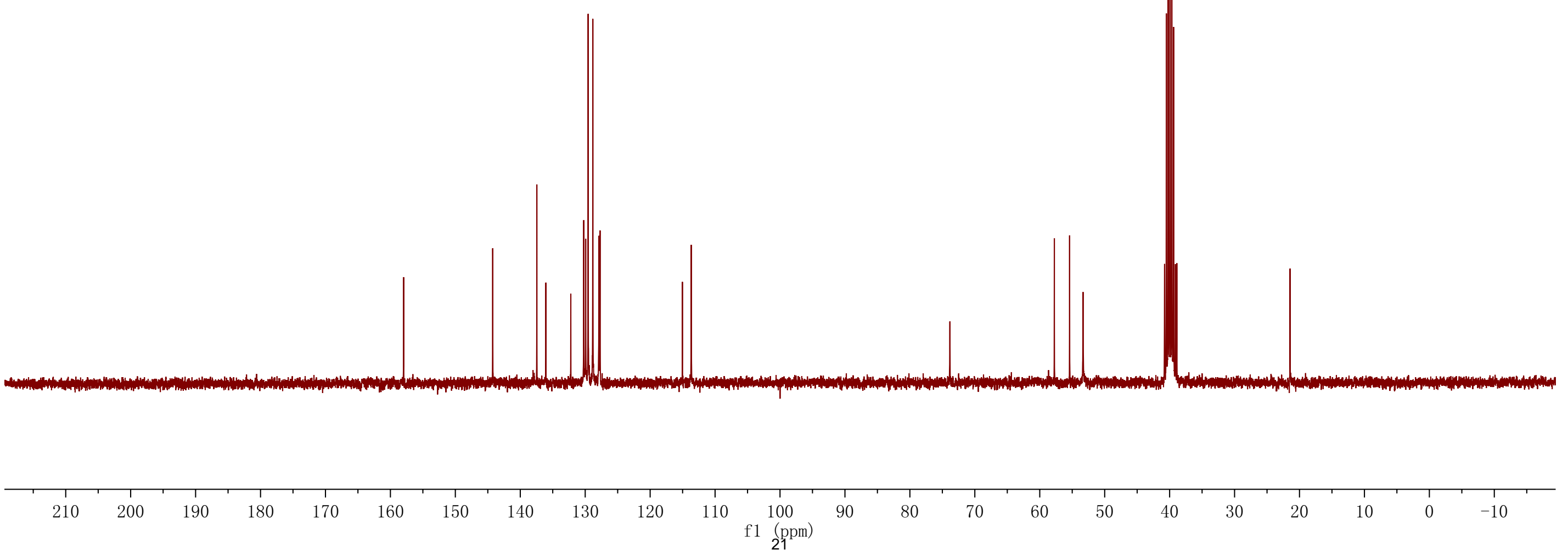


3ac, $400 \mathrm{MHz}, \mathrm{DMSO}-d_{6}$
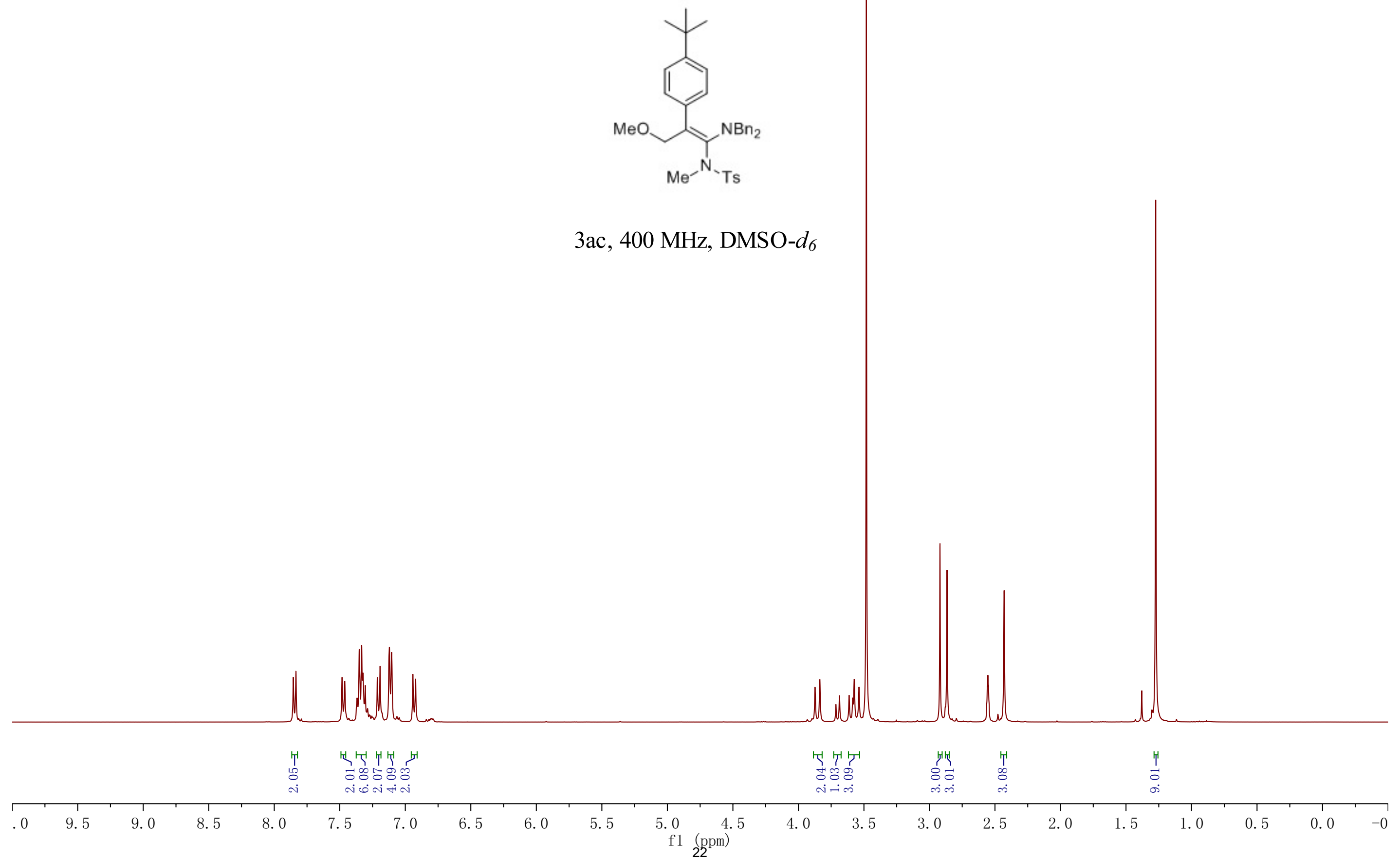


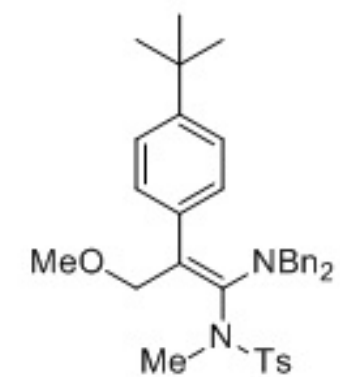

$3 \mathrm{ac}, 100 \mathrm{MHz}, \mathrm{DMSO}-d_{6}$

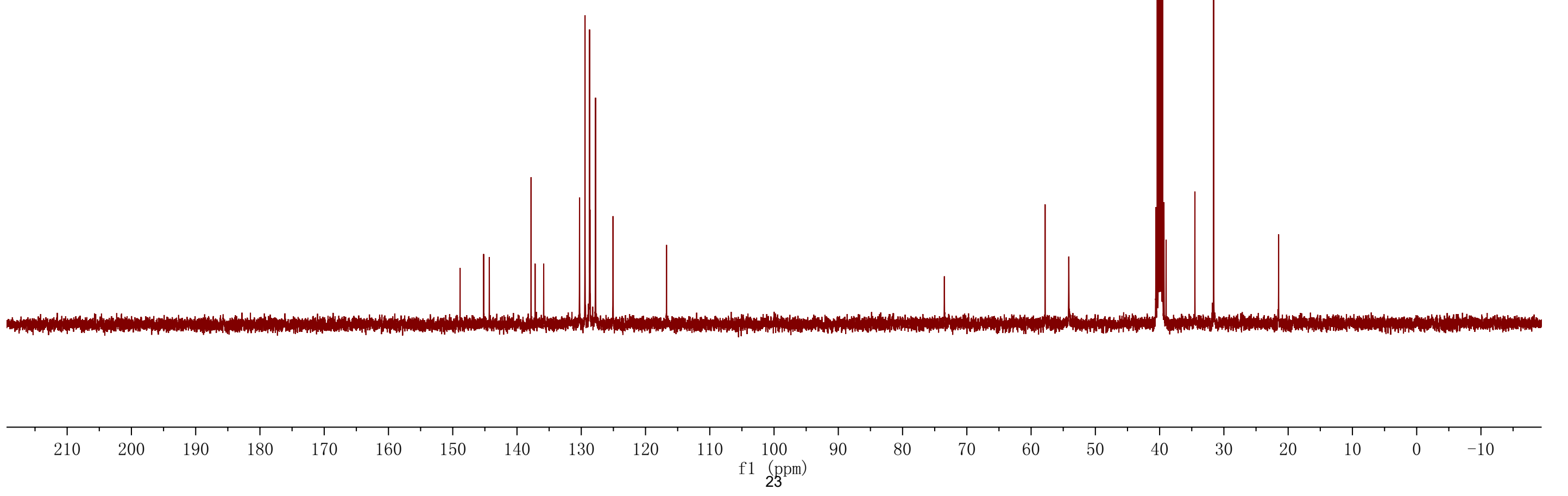




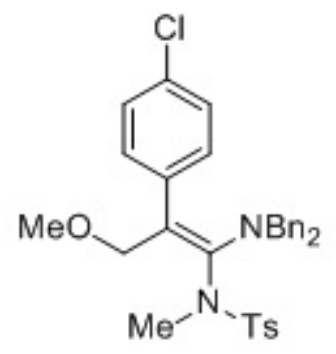

3ad, $400 \mathrm{MHz}, \mathrm{DMSO}-d_{6}$

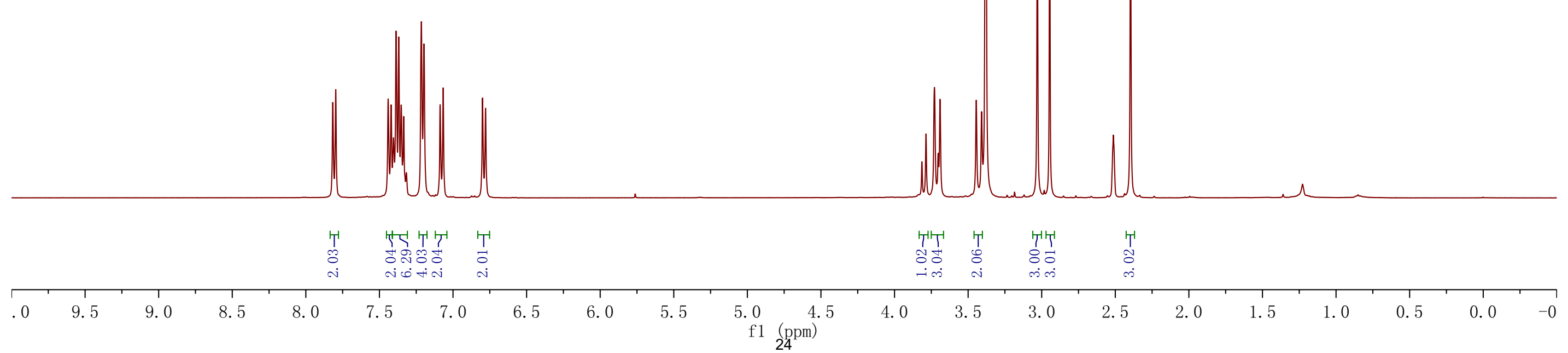


<smiles>COC/C(=C(\[18OH])N(C)C)c1ccc(Cl)cc1</smiles>

3ad, $100 \mathrm{MHz}$, DMSO- $d_{6}$

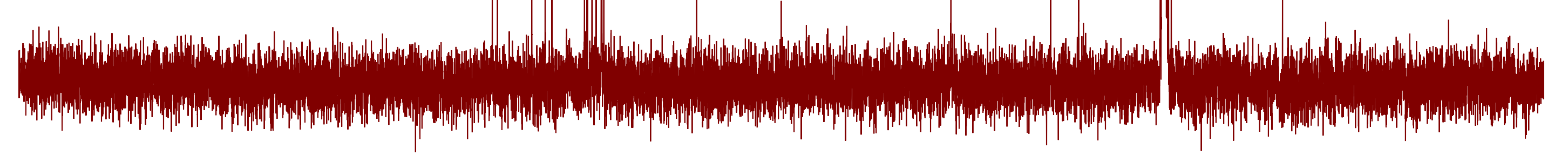

\begin{tabular}{|c|c|c|c|c|c|c|c|c|c|c|c|c|c|c|c|c|c|c|c|c|c|c|}
\hline 210 & 200 & 190 & 180 & 170 & 160 & 150 & 140 & 130 & 120 & 110 & $\begin{array}{c}100 \\
\mathrm{f} 1 \quad \begin{array}{l}(\mathrm{ppm}) \\
25\end{array}\end{array}$ & 90 & 80 & 70 & 60 & 50 & 40 & 30 & 20 & 10 & 0 & -10 \\
\hline
\end{tabular}




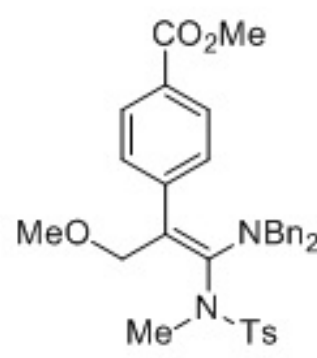

3ae, $400 \mathrm{MHz}, \mathrm{DMSO}-d_{6}$

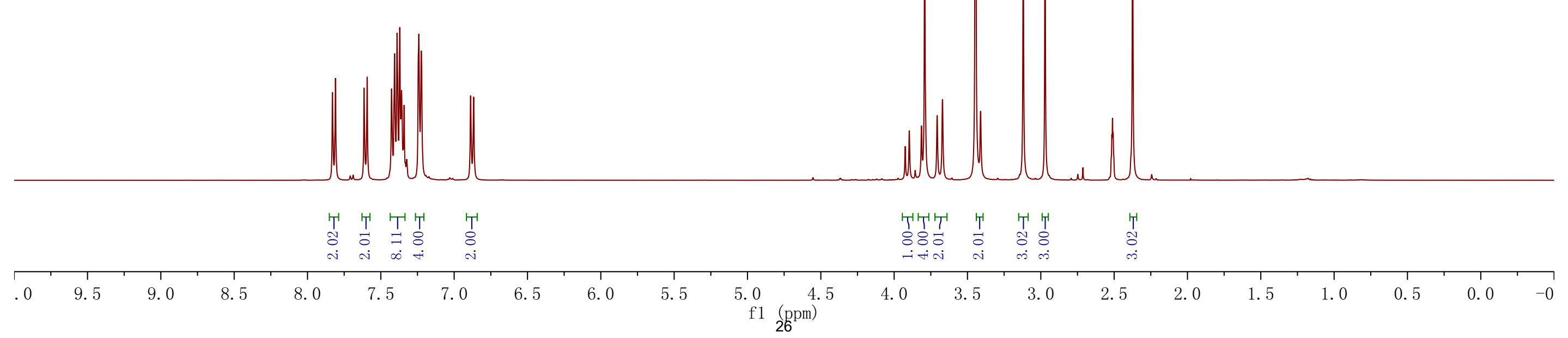




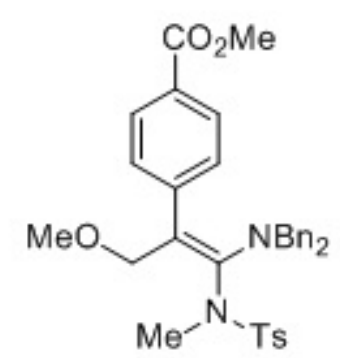

3ae, $75 \mathrm{MHz}$, DMSO- $d_{6}$

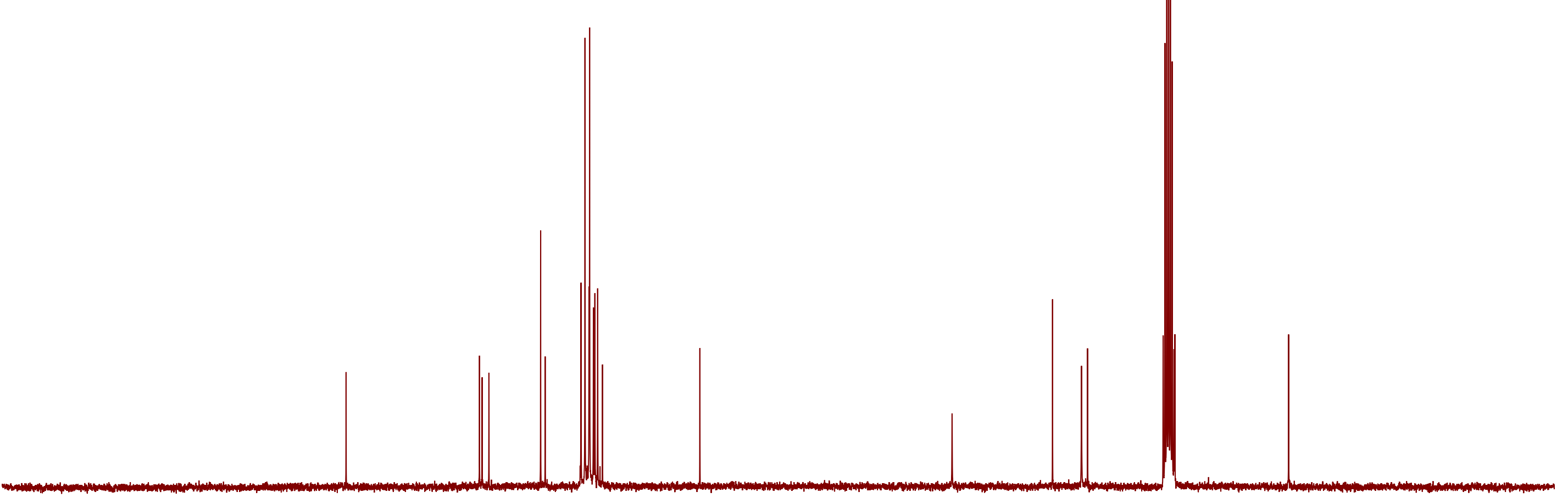

\begin{tabular}{|c|c|c|c|c|c|c|c|c|c|c|c|c|c|c|c|c|c|c|c|c|c|c|}
\hline 210 & $\begin{array}{c}1 \\
200\end{array}$ & $\begin{array}{c}1 \\
190\end{array}$ & $\begin{array}{c}1 \\
180\end{array}$ & $\begin{array}{c}1 \\
170\end{array}$ & $\begin{array}{c}1 \\
160\end{array}$ & $\begin{array}{c}1 \\
150\end{array}$ & $\begin{array}{c}1 \\
140\end{array}$ & $\begin{array}{c}1 \\
130\end{array}$ & $\begin{array}{c}1 \\
120\end{array}$ & 110 & $\begin{array}{c}100 \\
\mathrm{f} 1 \quad(\mathrm{ppm}) \\
27^{1}\end{array}$ & 90 & 80 & $\begin{array}{c}1 \\
70\end{array}$ & 60 & $\begin{array}{c}1 \\
50\end{array}$ & $\begin{array}{l}1 \\
40\end{array}$ & $\begin{array}{l}1 \\
30\end{array}$ & $\begin{array}{l}1 \\
20\end{array}$ & 10 & $\begin{array}{l}1 \\
0\end{array}$ & $\begin{array}{c}1 \\
-10\end{array}$ \\
\hline
\end{tabular}




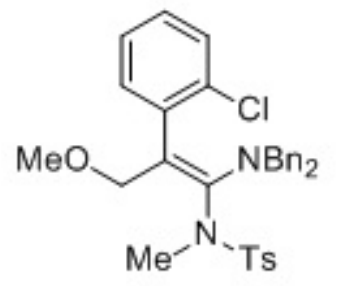

3af, $400 \mathrm{MHz}$, DMSO- $d_{6}$

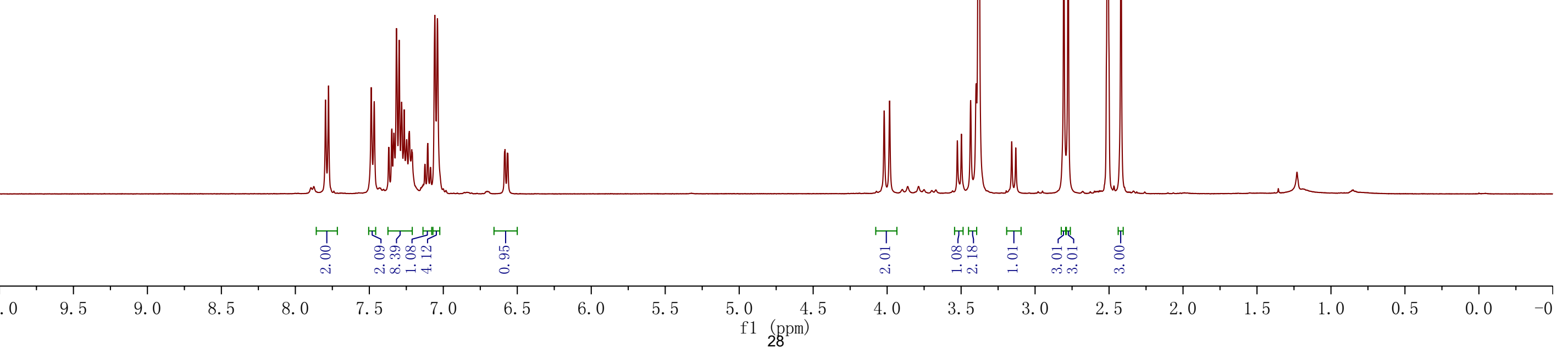



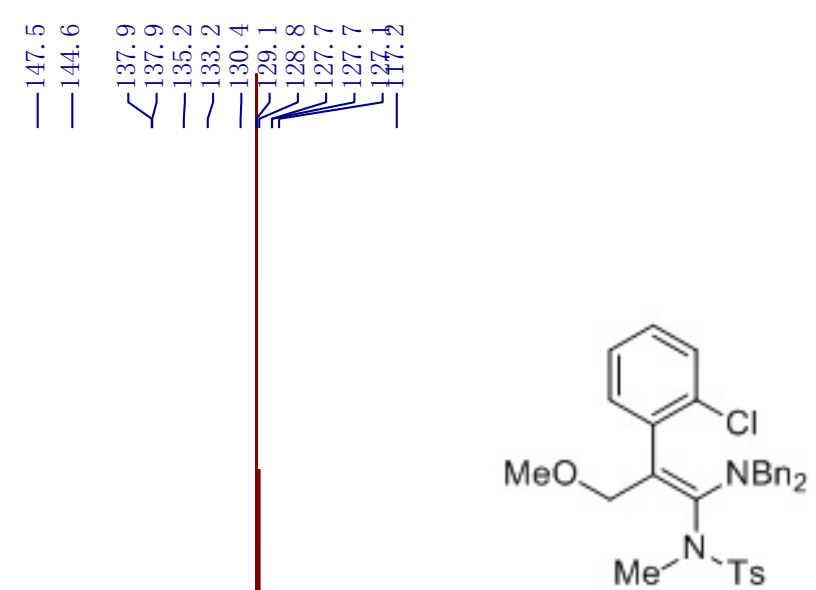

3af, $100 \mathrm{MHz}, \mathrm{DMSO}-d_{6}$

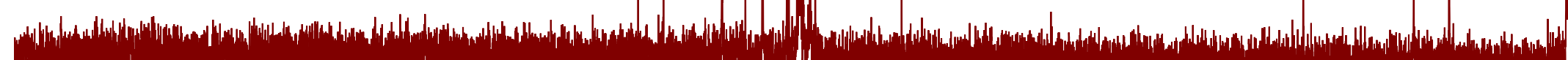

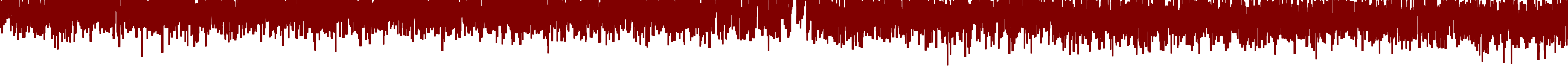

\begin{tabular}{|c|c|c|c|c|c|c|c|c|c|c|c|c|c|c|c|c|c|c|c|c|c|c|}
\hline 210 & 200 & 190 & 180 & 170 & 160 & 150 & 140 & 130 & 120 & 110 & 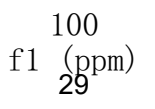 & 90 & 80 & 70 & 60 & 50 & 40 & 30 & 20 & 10 & 0 & -10 \\
\hline
\end{tabular}




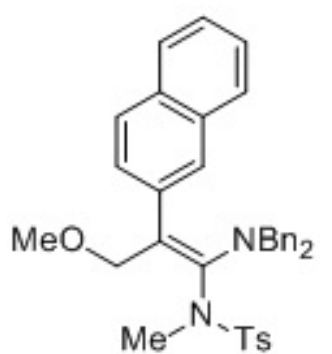

3ag, $400 \mathrm{MHz}, \mathrm{DMSO}-d_{6}$
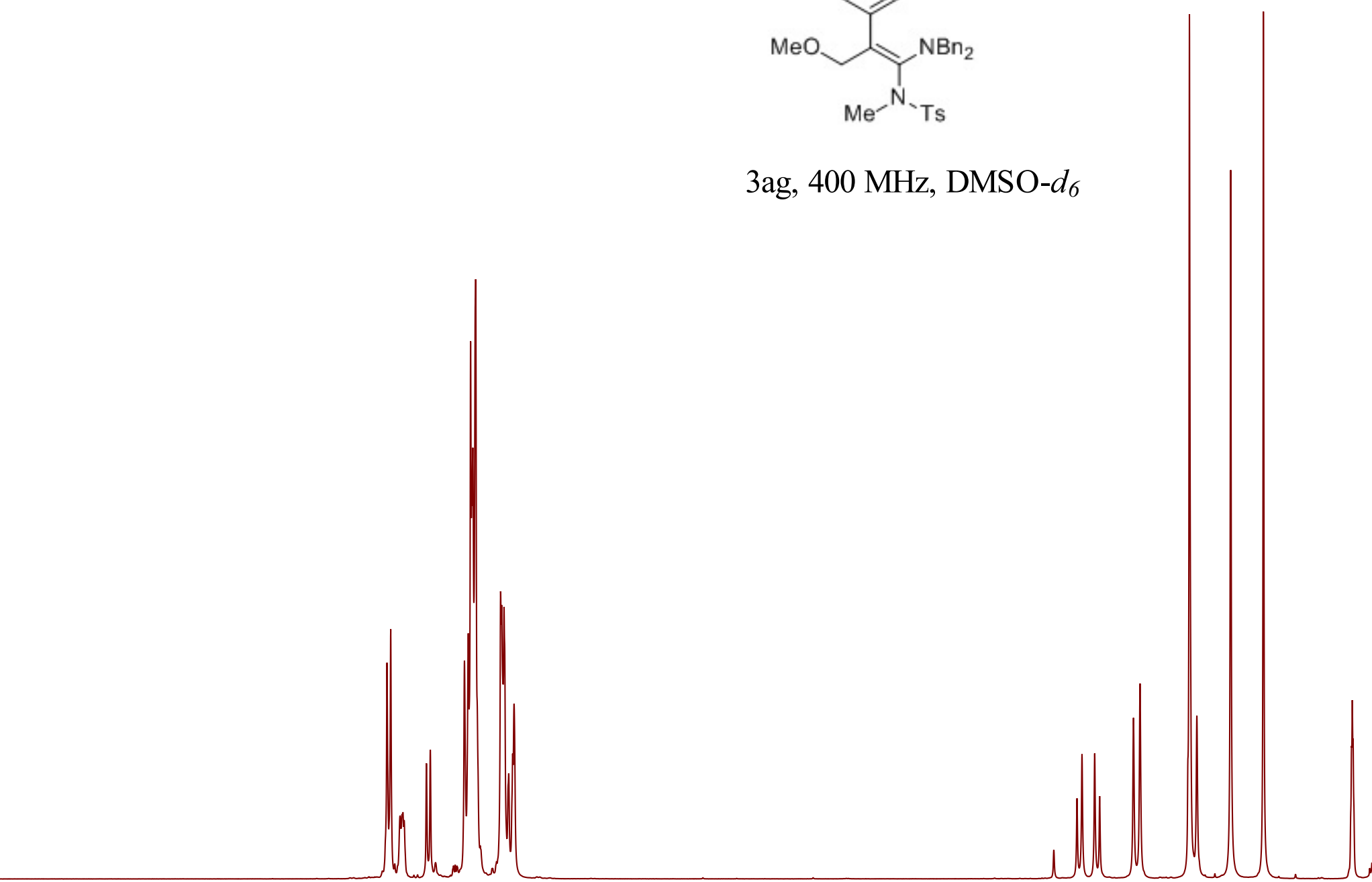

T'T T' Tा

8.

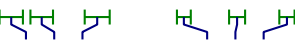

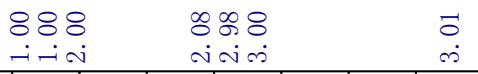

个

$\begin{array}{lll}9.0 & 8.5 & 8.0\end{array}$

$7.5 \quad 7.0$

6.5

6. 0

5.5

f1 (ppm)

3. 5.0

$2.5 \quad 2.0$

$0 \quad 1.5$ 


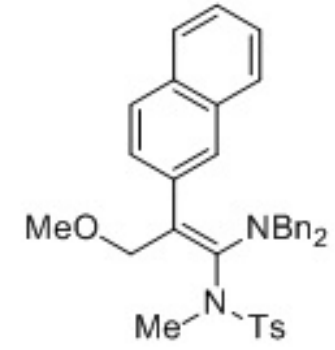

3ag, $75 \mathrm{MHz}$, DMSO-d 6

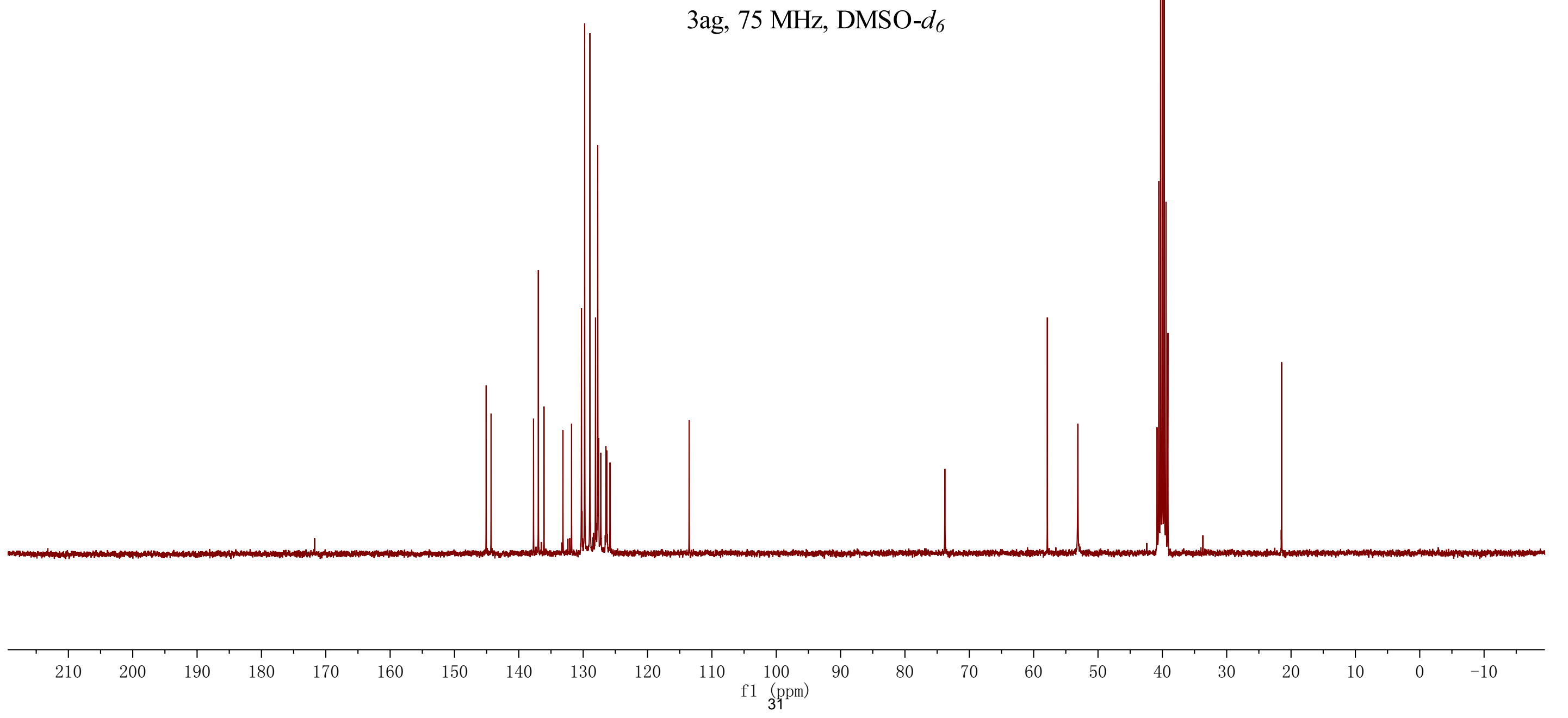




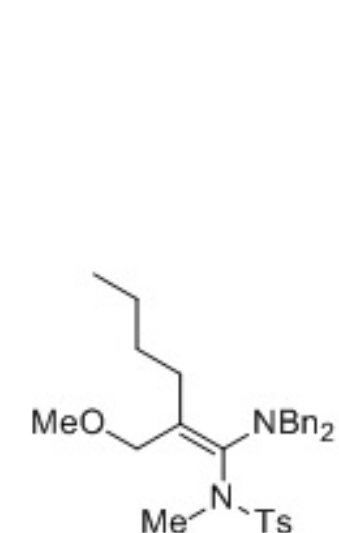

3ah, $300 \mathrm{MHz}$, DMSO- $d_{6}$

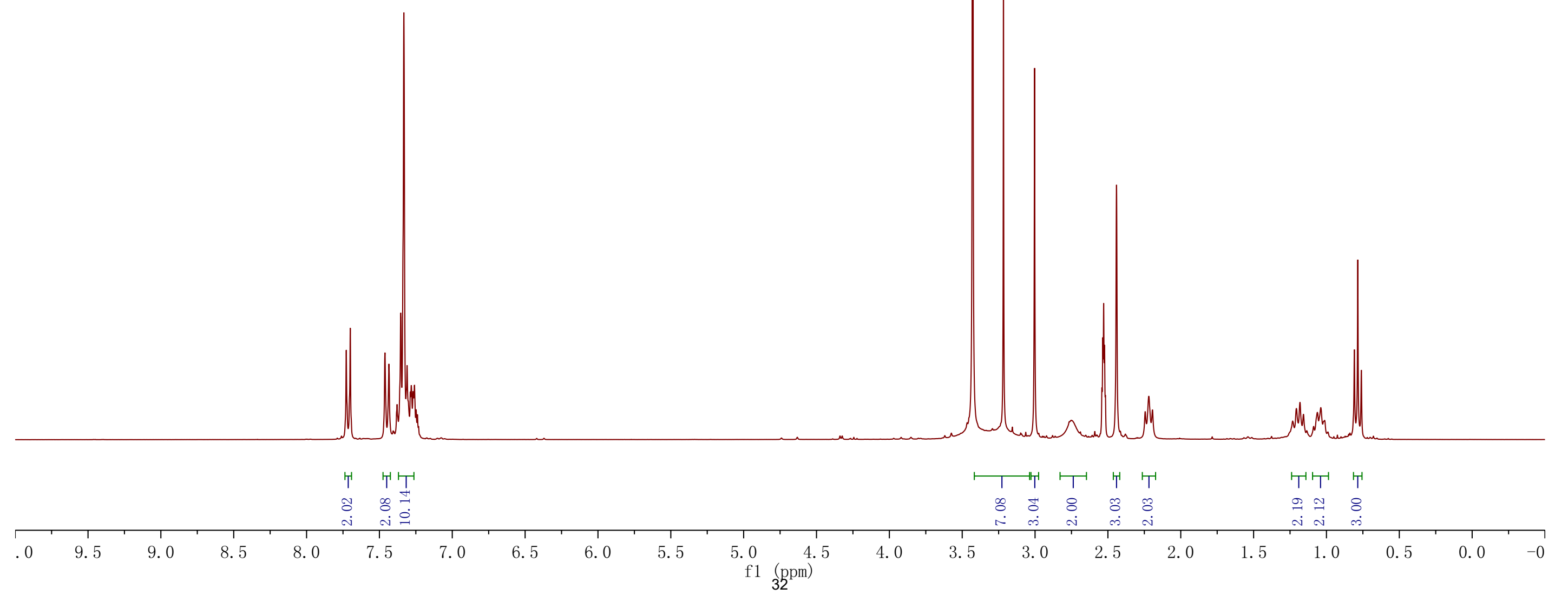




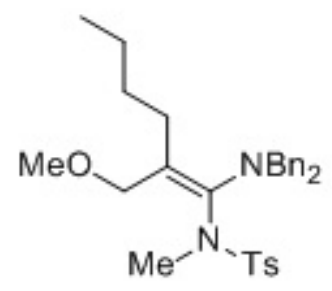

3ah, $75 \mathrm{MHz}$, DMSO- $d_{6}$

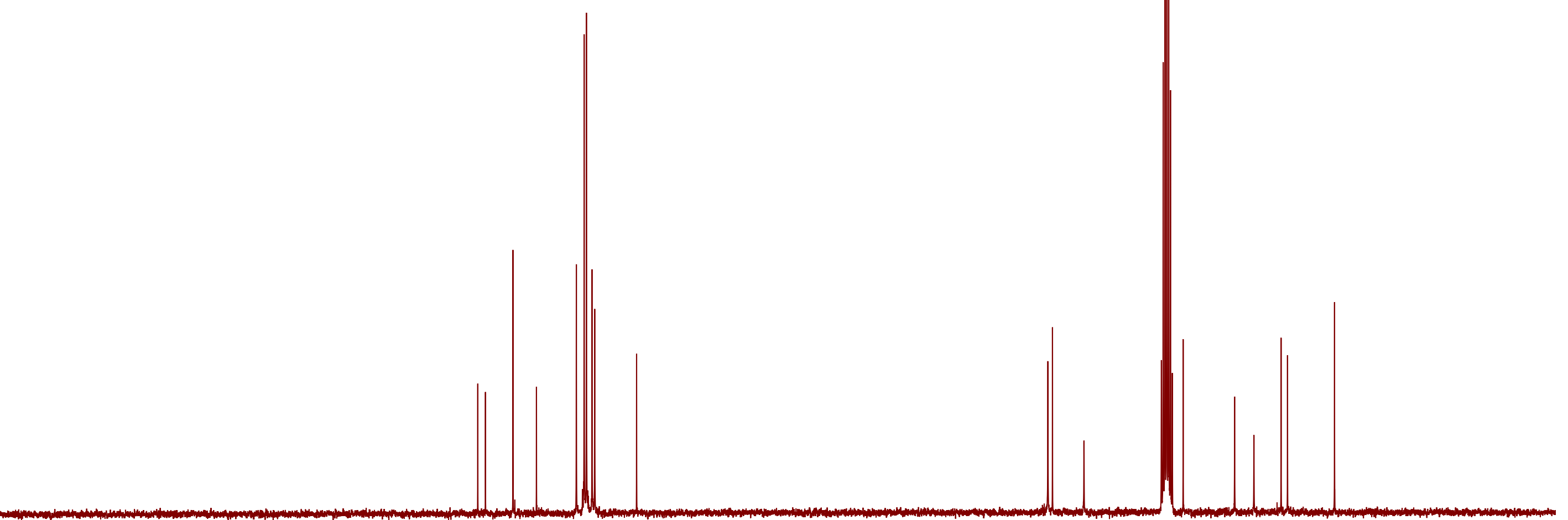

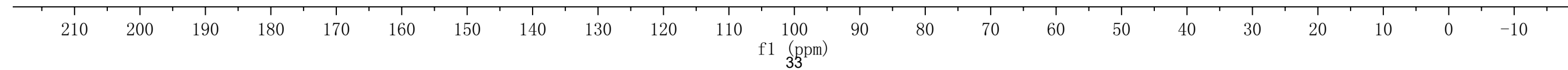




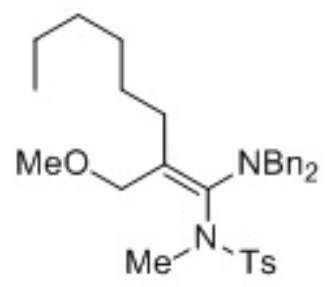

3ai, $400 \mathrm{MHz}, \mathrm{DMSO}-d_{6}$

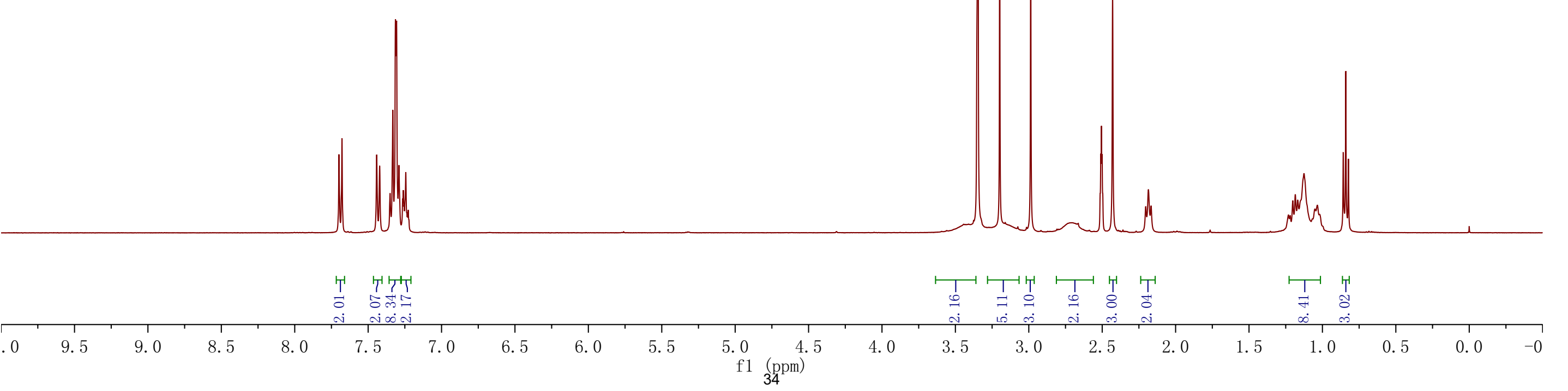




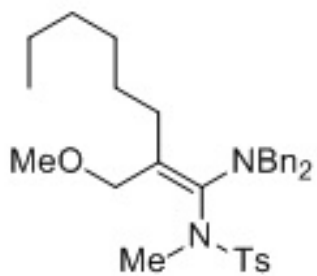

3ai, $75 \mathrm{MHz}, \mathrm{DMSO}-d_{6}$

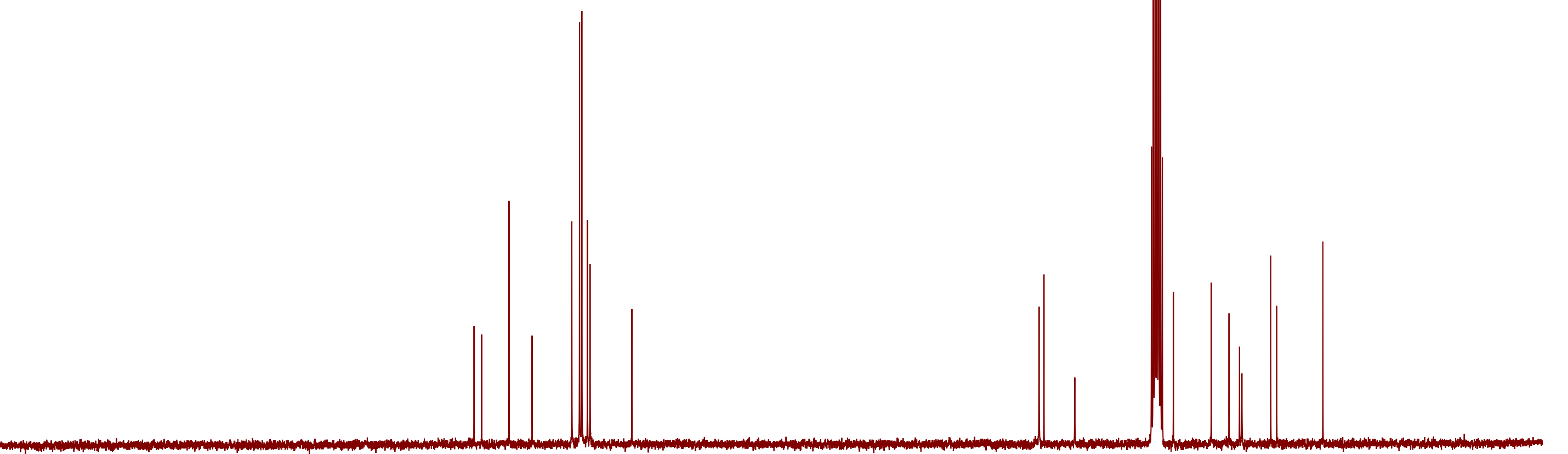

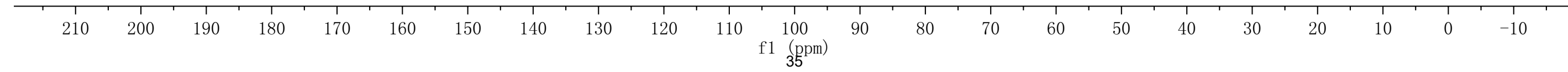




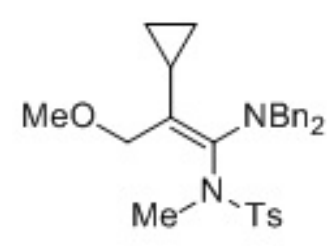

3aj, $400 \mathrm{MHz}$, DMSO- $d_{6}$

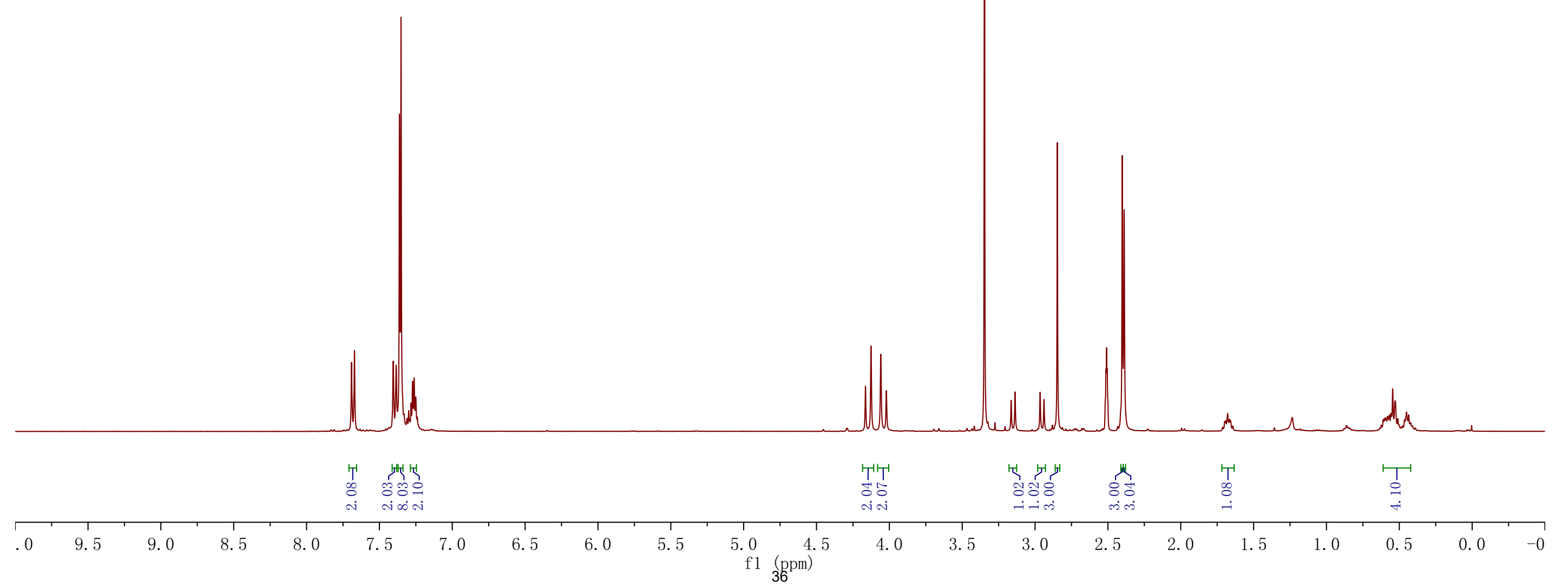




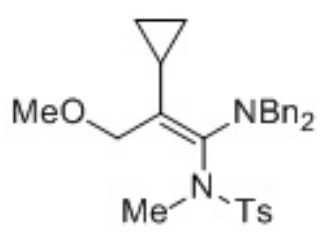

3aj, $75 \mathrm{MHz}$, DMSO- $d_{6}$

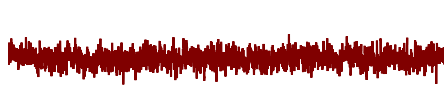

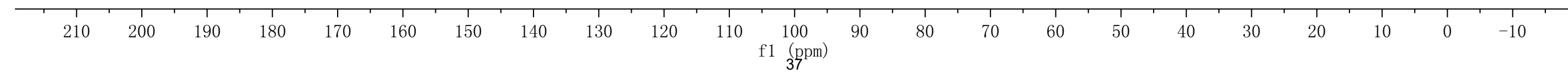




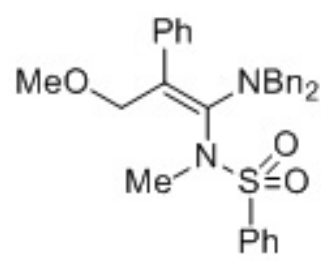

3ak, $400 \mathrm{MHz}, \mathrm{DMSO}-d_{6}$

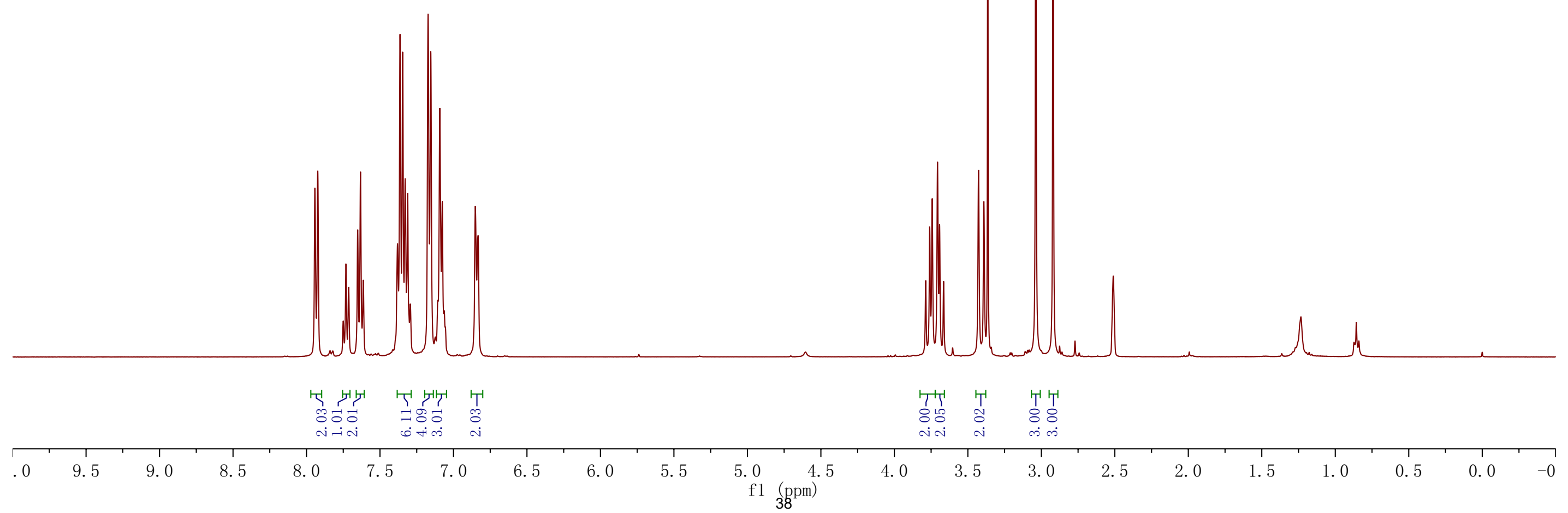




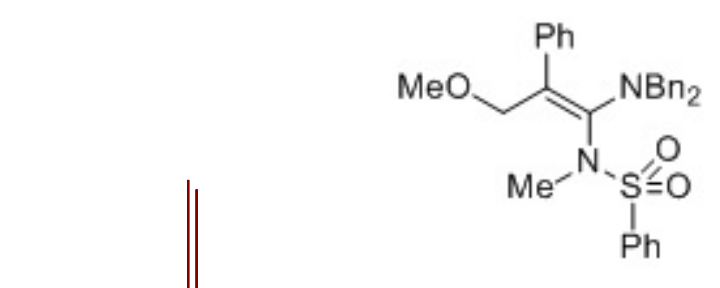

3ak, $75 \mathrm{MHz}$, DMSO- $d_{6}$

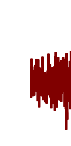
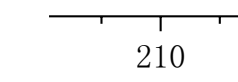

$200 \quad 190$

180

170

160

150

140

130

120

110

100

90

1
$90 \quad 80$

단

60 50 


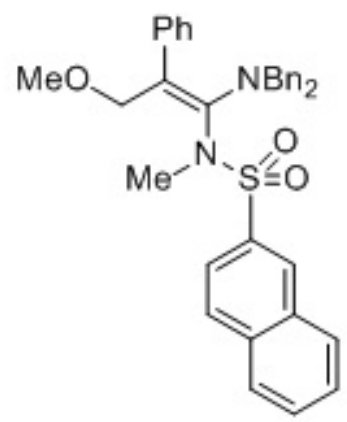

3al, $400 \mathrm{MHz}, \mathrm{DMSO}-d_{6}$

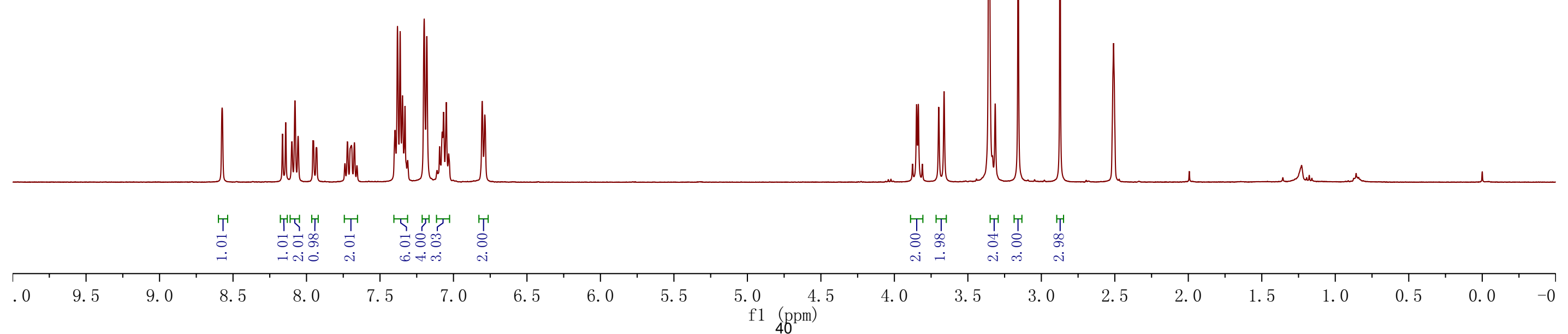




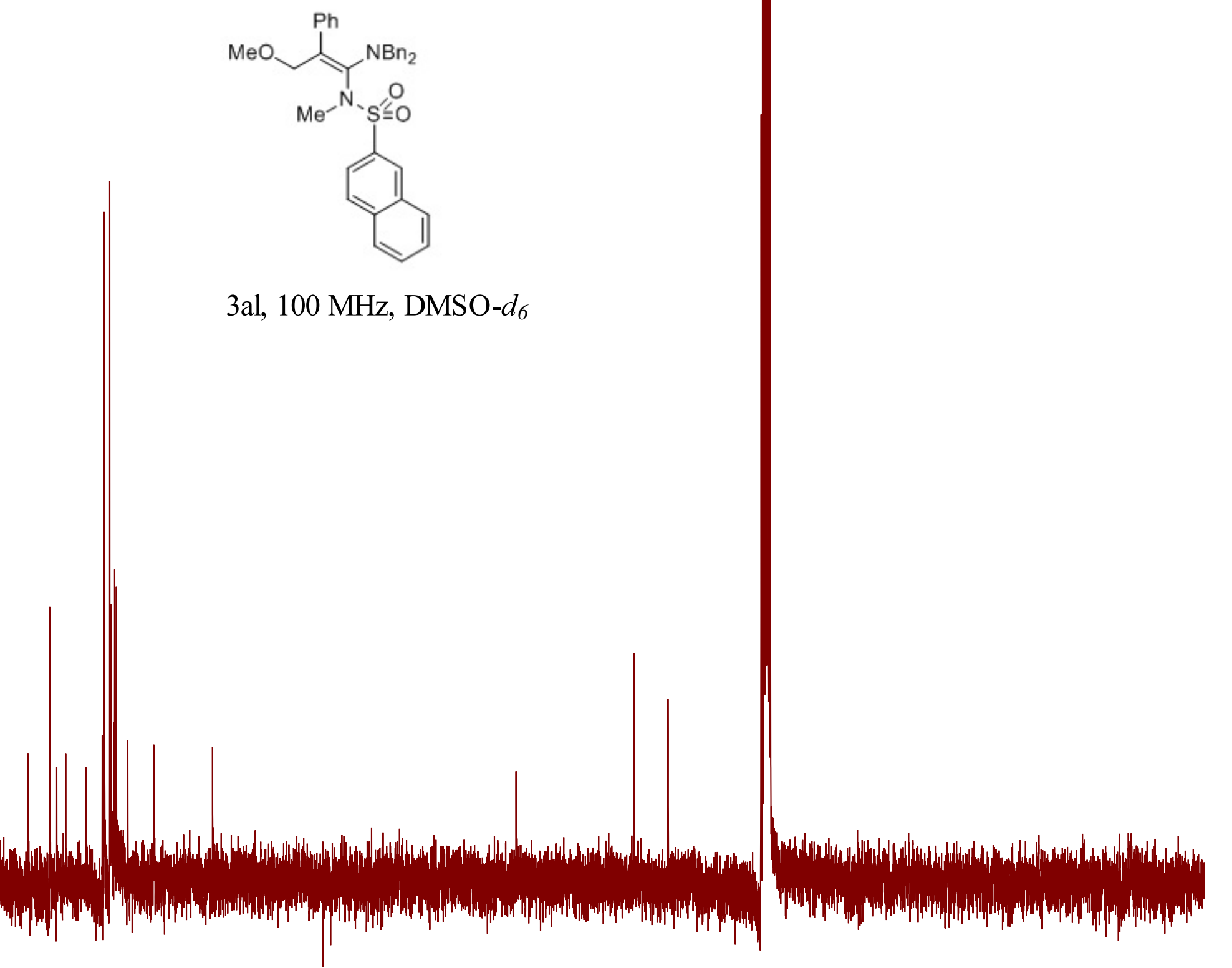

3al, $100 \mathrm{MHz}$, DMSO- $d_{6}$

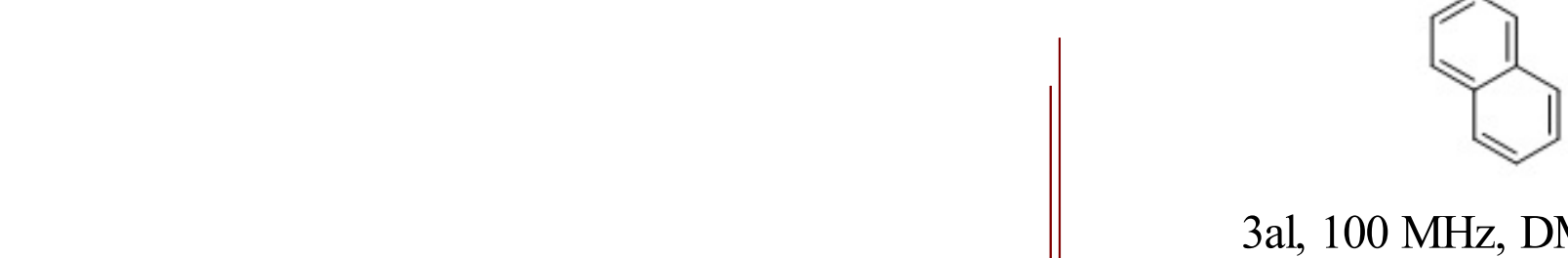

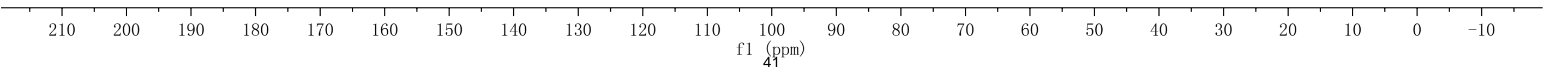




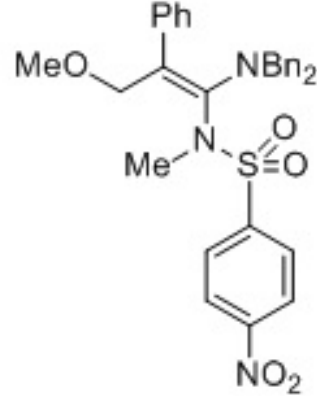

3am, 400 MHz, DMSO- $d_{6}$

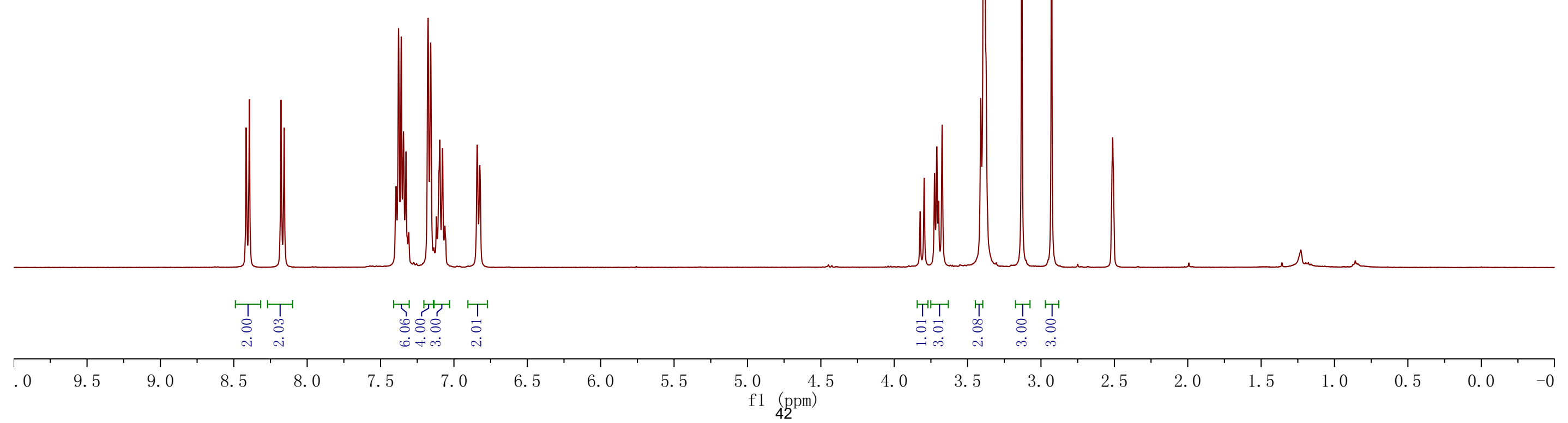




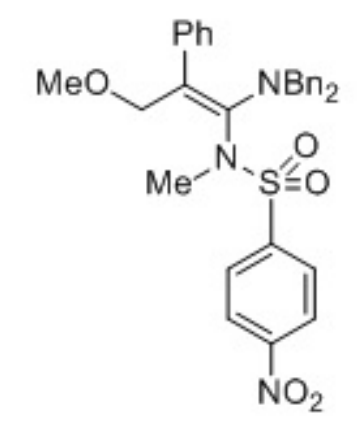

3am, 100 MHz, DMSO-d 6

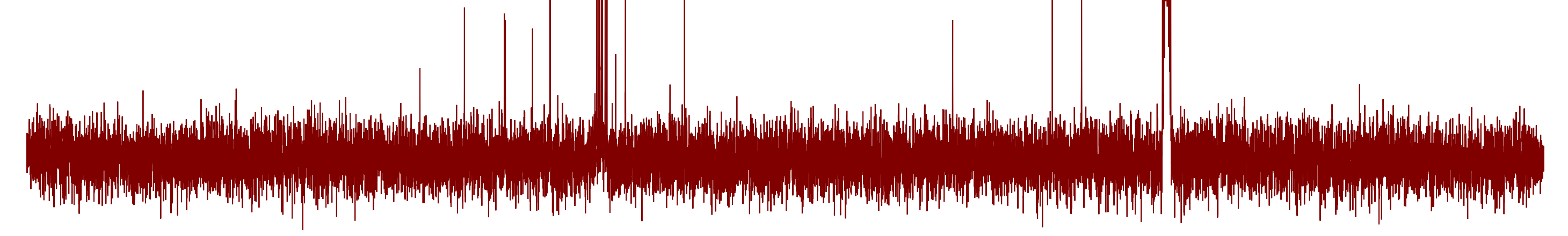

\begin{tabular}{|c|c|c|c|c|c|c|c|c|c|c|c|c|c|c|c|c|c|c|c|c|c|c|}
\hline 210 & 200 & 190 & 180 & 170 & 160 & 150 & 140 & 130 & 120 & 110 & $\begin{array}{c}100 \\
\left.\mathrm{f} 1 \begin{array}{c}(\mathrm{pm}) \\
43\end{array}\right)\end{array}$ & 90 & 80 & 70 & 60 & 50 & 40 & 30 & 20 & 10 & 0 & -10 \\
\hline
\end{tabular}


列<smiles>COC/C(=C(\[18OH])c1ccccc1)N([14CH3])S(=O)(=O)c1ccc(C(F)(F)F)cc1</smiles>

3an, $400 \mathrm{MHz}$, DMSO-d 6
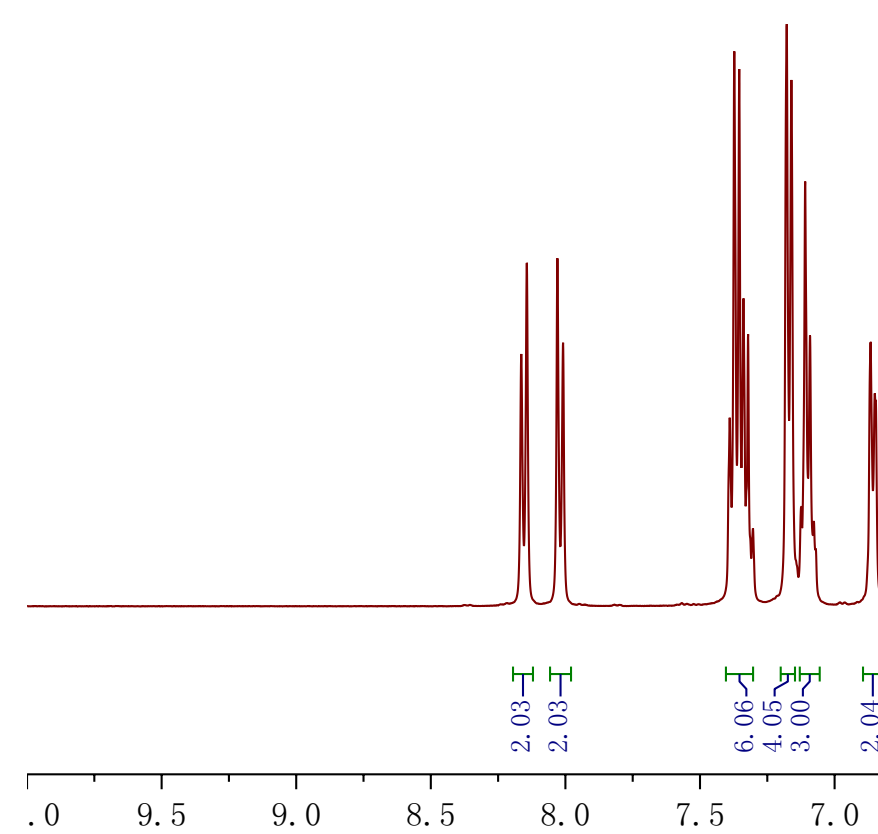

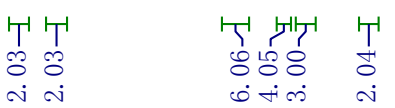

$1,1,0,0,0$

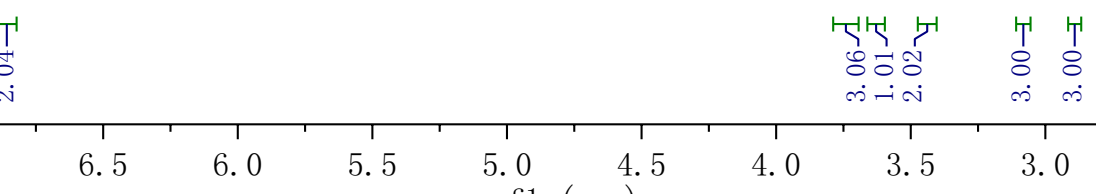
1 (ppm) 


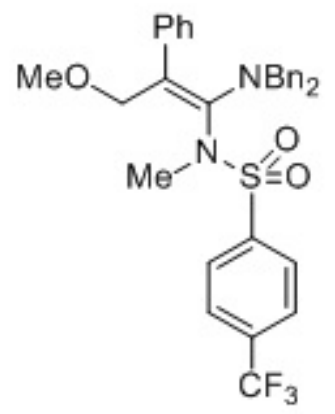

3an, $100 \mathrm{MHz}$, DMSO- $d_{6}$

\begin{tabular}{|c|c|c|c|c|c|c|c|c|c|c|c|c|c|c|c|c|c|c|c|c|c|c|}
\hline 210 & $\begin{array}{l}1 \\
200\end{array}$ & $\begin{array}{l}1 \\
190\end{array}$ & $\begin{array}{c}1 \\
180\end{array}$ & $\begin{array}{c}1 \\
170\end{array}$ & $\begin{array}{l}160 \\
160\end{array}$ & $\begin{array}{l}1 \\
150\end{array}$ & $\begin{array}{c}1 \\
140\end{array}$ & $\begin{array}{c}1 \\
130\end{array}$ & $\begin{array}{l}1 \\
120\end{array}$ & $\begin{array}{l}110 \\
110\end{array}$ & $\begin{array}{c}100 \\
\text { f1 } \\
\\
45\end{array}$ & $\begin{array}{l}1 \\
90\end{array}$ & $\begin{array}{l}1 \\
80\end{array}$ & $\begin{array}{l}1 \\
70\end{array}$ & 60 & $\begin{array}{l}1 \\
50\end{array}$ & $\begin{array}{l}1 \\
40\end{array}$ & $\begin{array}{l}1 \\
30\end{array}$ & $\begin{array}{l}1 \\
20\end{array}$ & 10 & $\begin{array}{l}1 \\
0\end{array}$ & $\begin{array}{c}1 \\
-10\end{array}$ \\
\hline
\end{tabular}




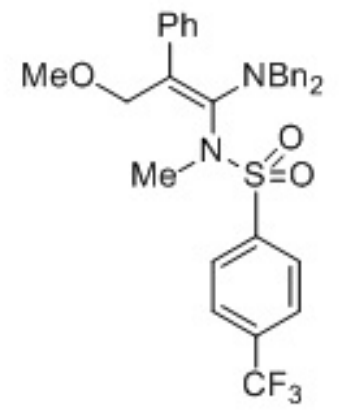

3an, $376 \mathrm{MHz}, \mathrm{DMSO}-d_{6}$

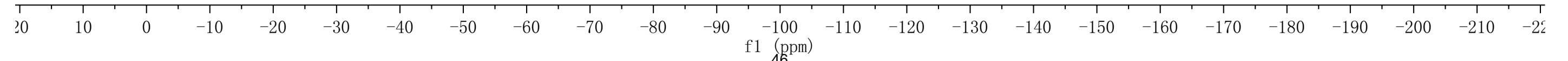




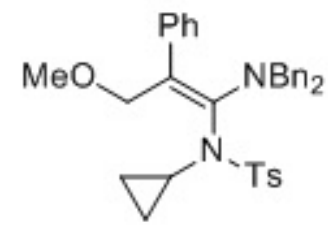

3ao, $400 \mathrm{MHz}$, DMSO- $d_{6}$

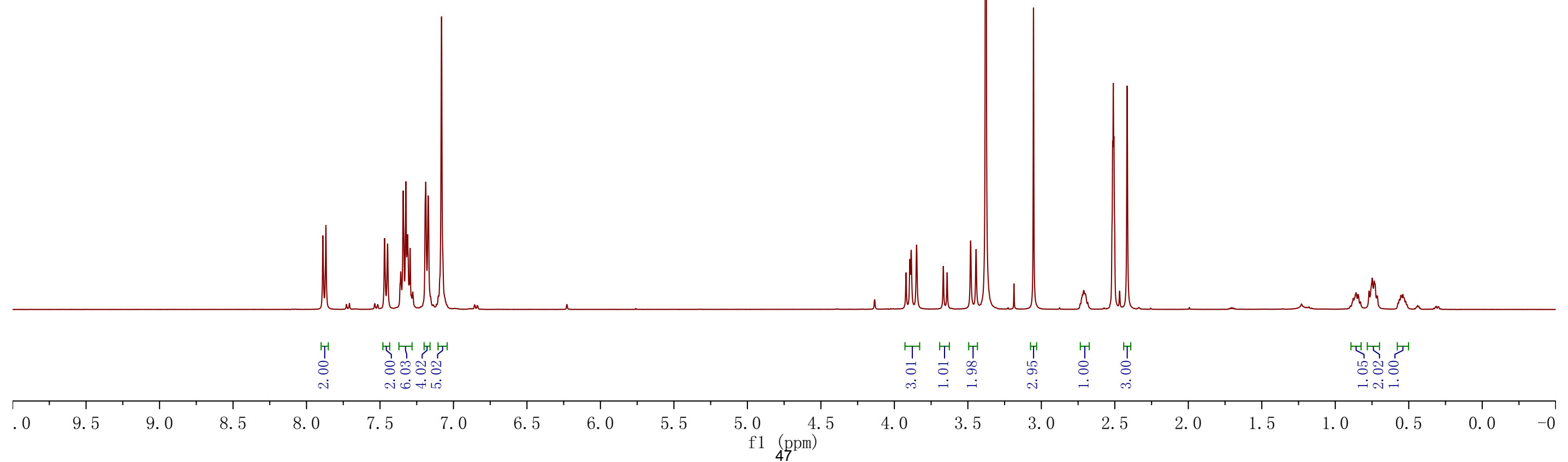




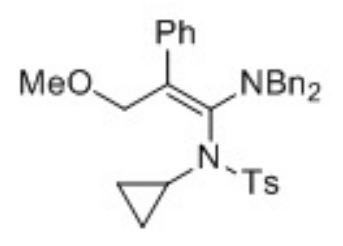

3ao, $75 \mathrm{MHz}, \mathrm{DMSO}-d_{6}$

\begin{tabular}{|c|c|c|c|c|c|c|c|c|c|c|c|c|c|c|c|c|c|c|c|c|c|c|}
\hline 210 & 200 & 190 & 180 & 170 & 160 & 150 & 140 & 130 & 120 & 110 & $\begin{array}{c}100 \\
\text { f1 } \quad(\mathrm{ppm}) \\
48\end{array}$ & 90 & 80 & 70 & 60 & 50 & 40 & 30 & 20 & 10 & 0 & -10 \\
\hline
\end{tabular}




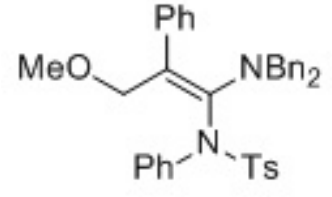

3ap, $400 \mathrm{MHz}$, DMSO- $d_{6}$
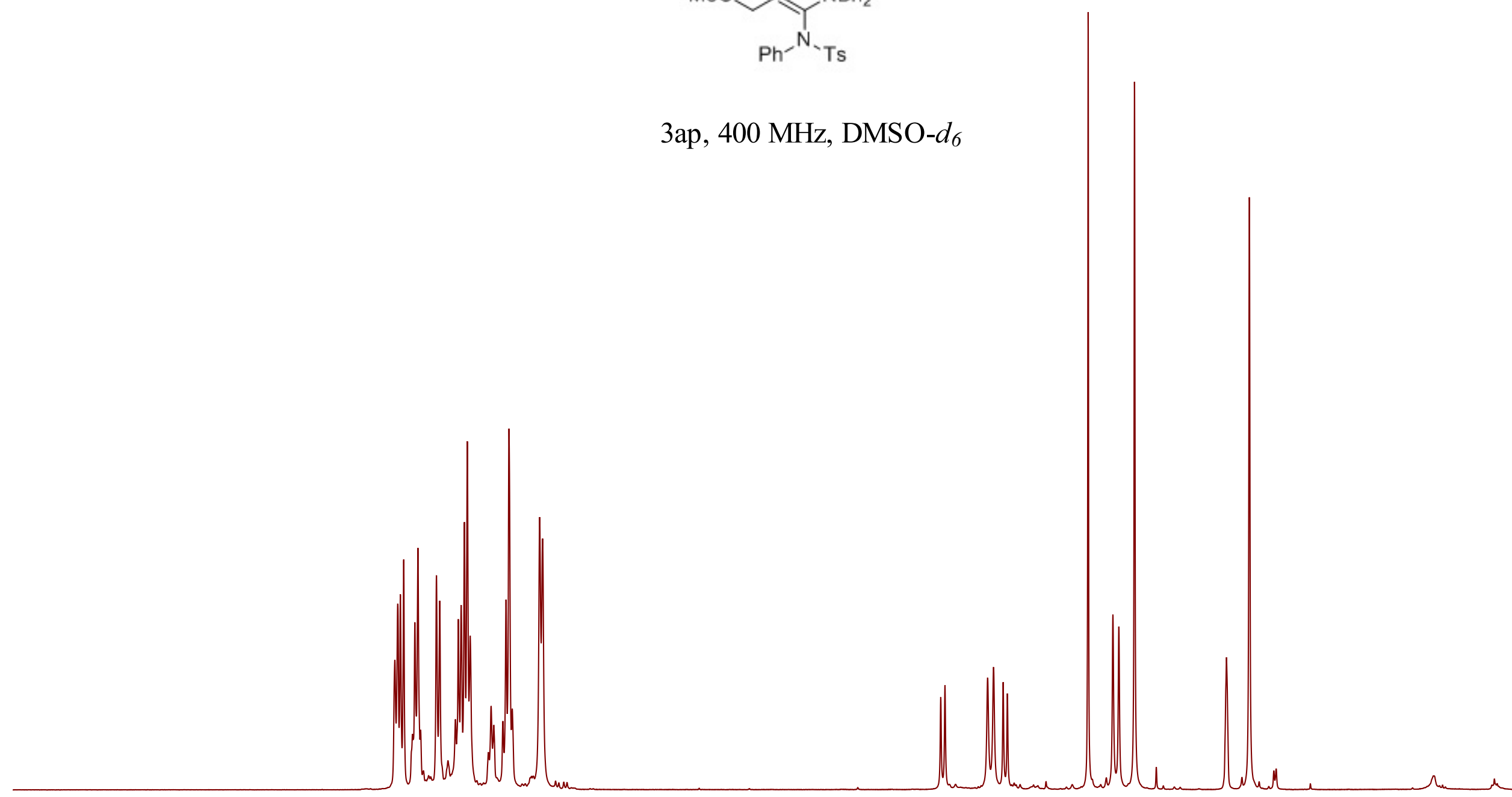

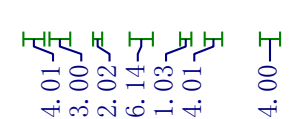
(t) $7.5 \quad 7.0 \quad 6.5$

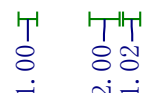
T' T
8 ชิ $T$
$\stackrel{1}{8}$
$\dot{\infty}$

$8.5 \quad 8.0$
$6.5 \quad 6.0$ 


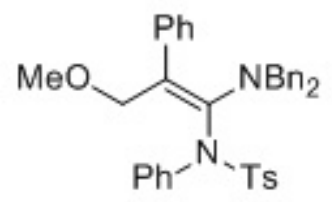

3ap, $75 \mathrm{MHz}$, DMSO- $d_{6}$
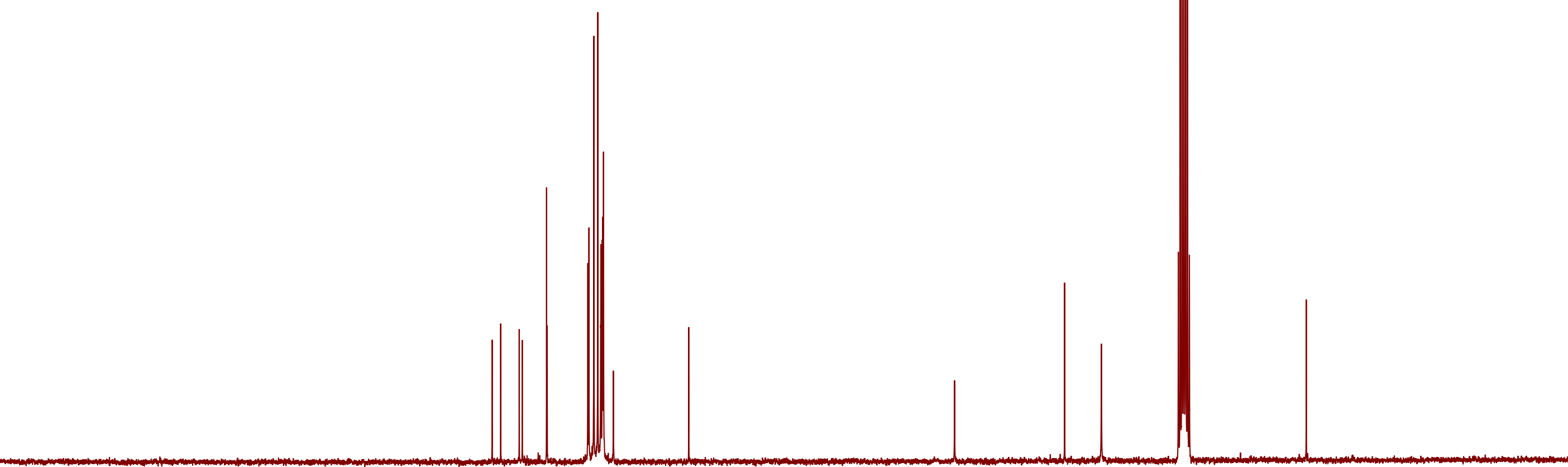

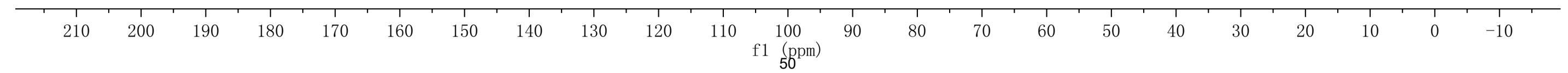




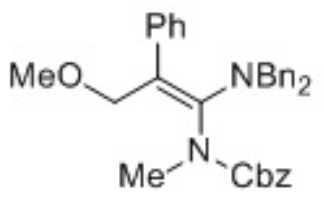

3aq, $400 \mathrm{MHz}, \mathrm{DMSO}-d_{6}$

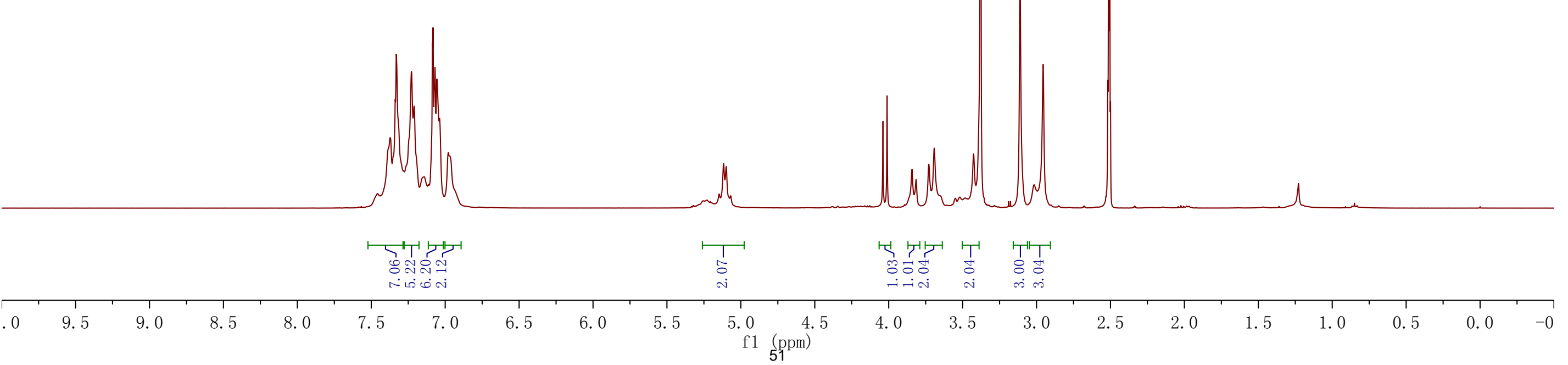




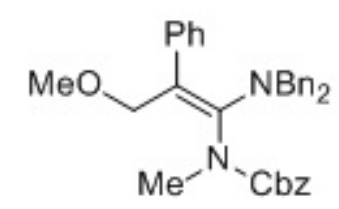

3aq, $100 \mathrm{MHz}, \mathrm{DMSO}-d_{6}$

\begin{tabular}{|c|c|c|c|c|c|c|c|c|c|c|c|c|c|c|c|c|c|c|c|c|c|c|}
\hline 210 & 200 & 190 & 180 & 170 & 160 & 150 & 140 & 130 & 120 & 110 & $\begin{array}{c}100 \\
\mathrm{f} 1 \stackrel{(\mathrm{pmm})}{52}\end{array}$ & 90 & 80 & 70 & 60 & 50 & 40 & 30 & 20 & 10 & 0 & -10 \\
\hline
\end{tabular}




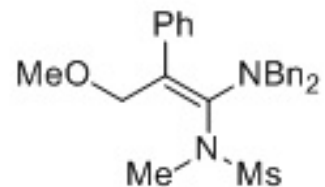

3ar, $400 \mathrm{MHz}, \mathrm{DMSO}-d_{6}$
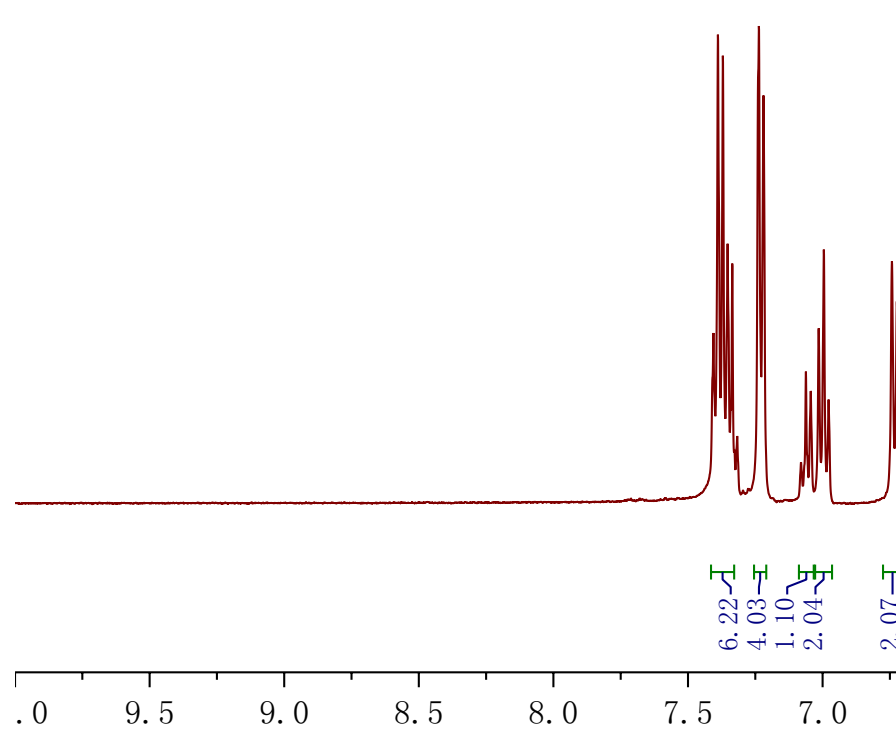

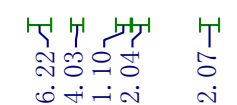

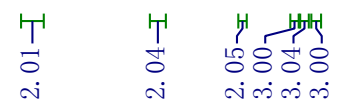




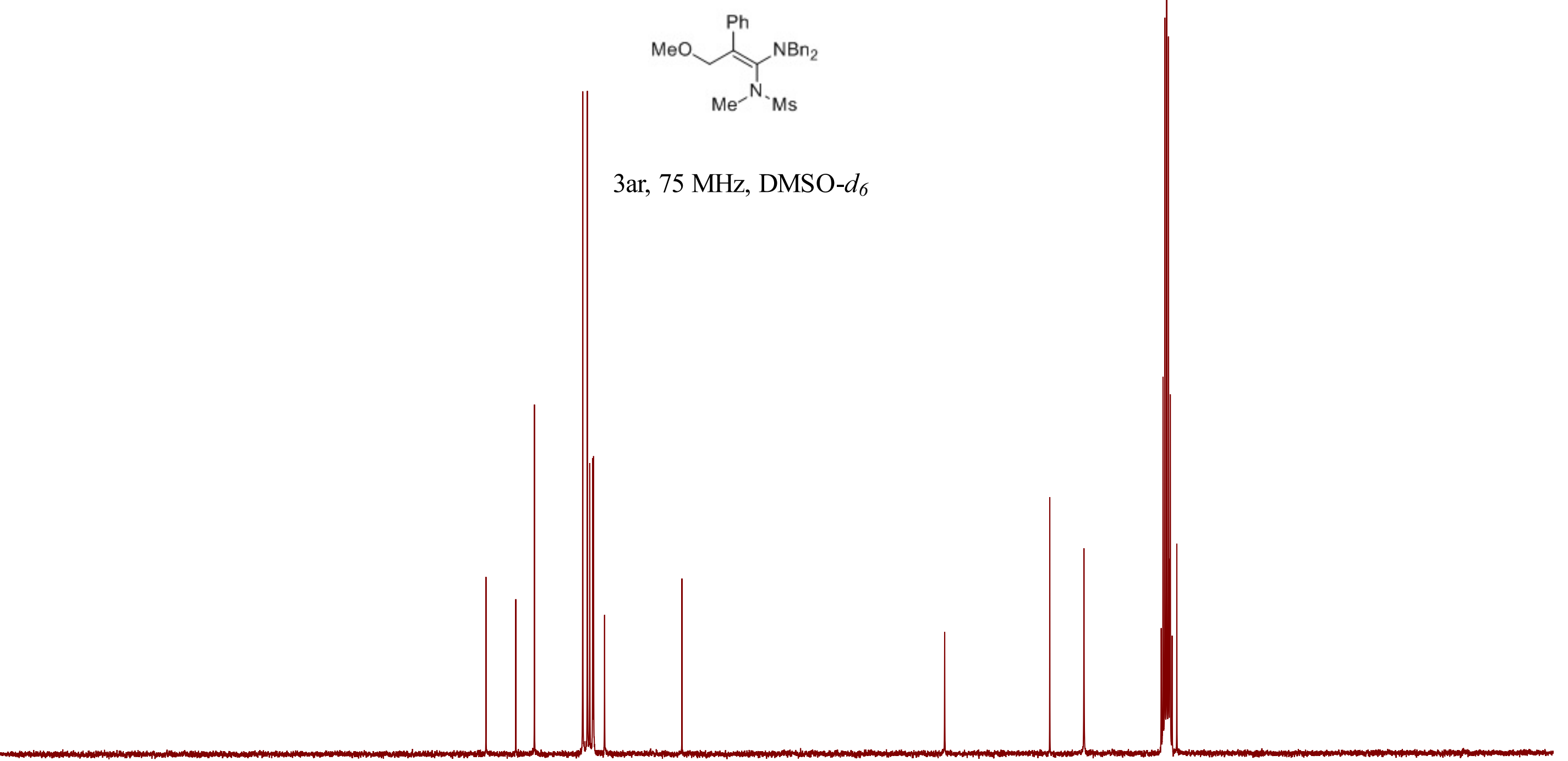

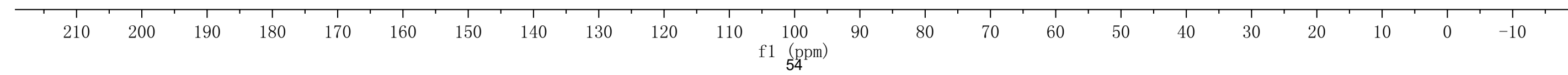




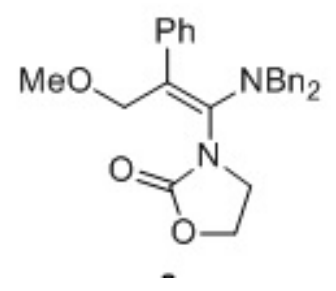

3as, $400 \mathrm{MHz}$, DMSO- $d_{6}$

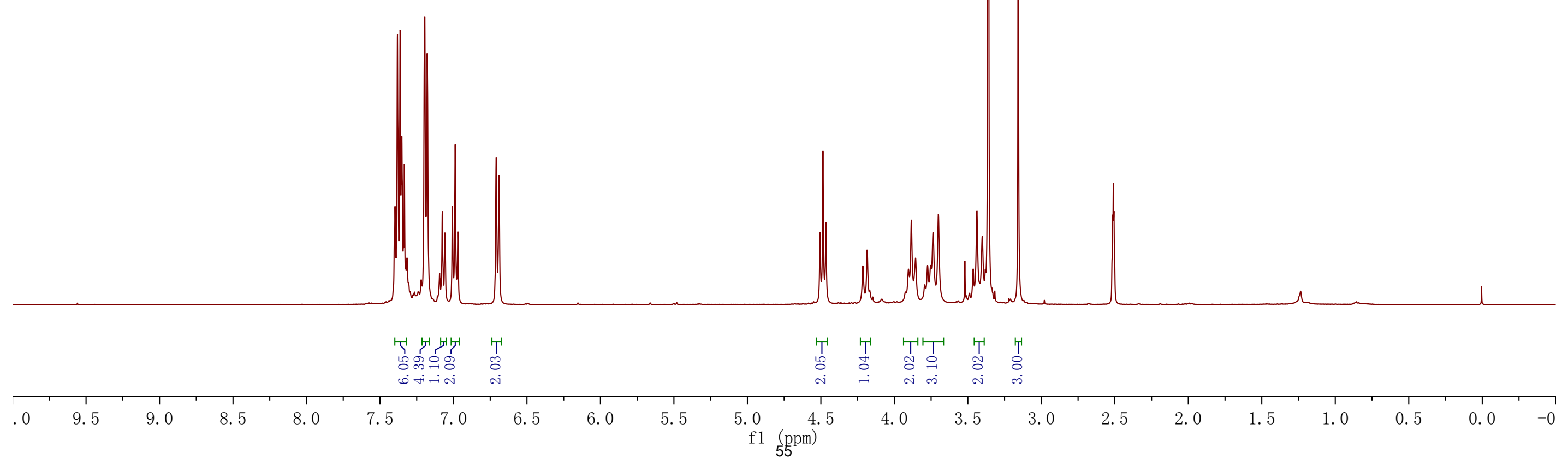


l|

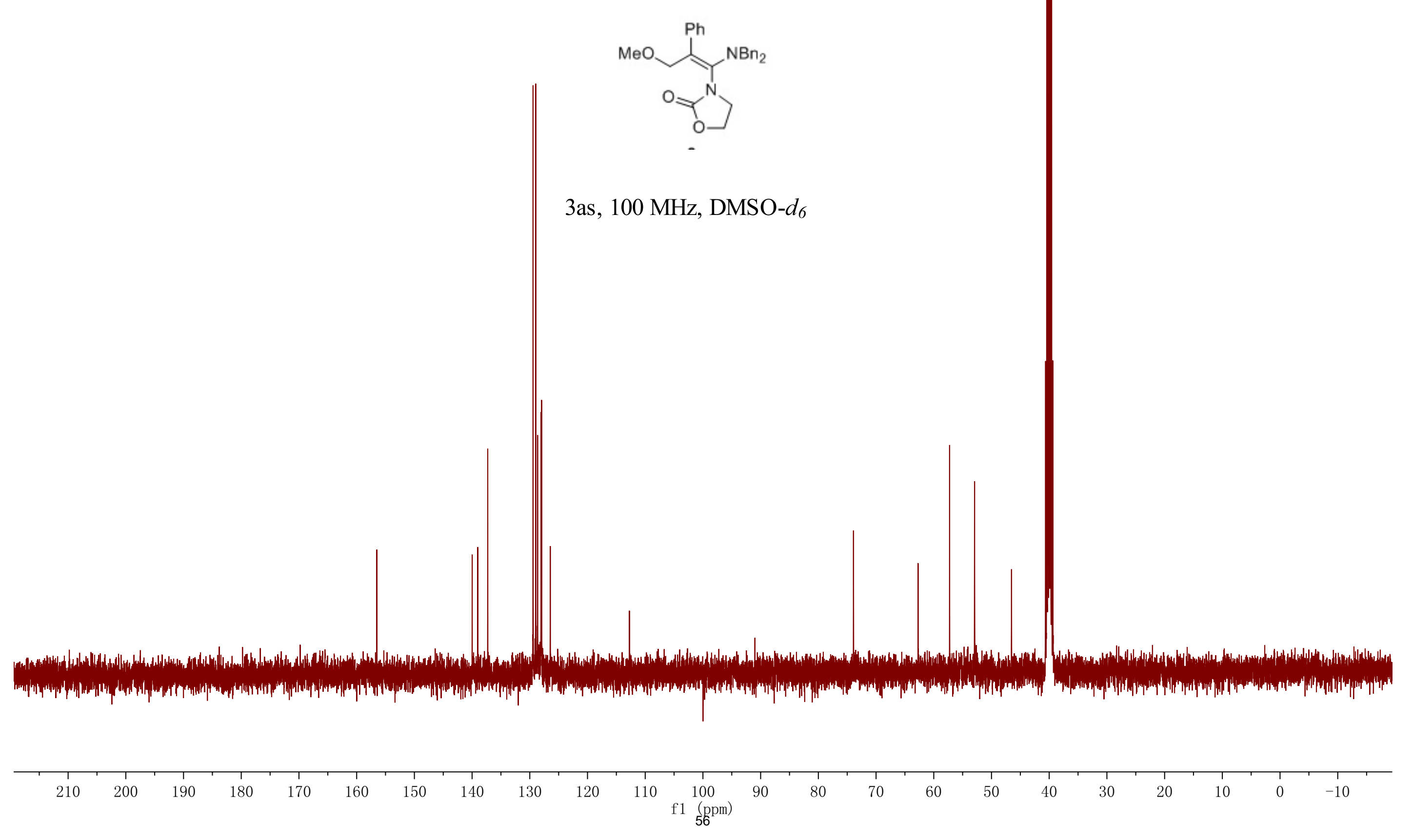




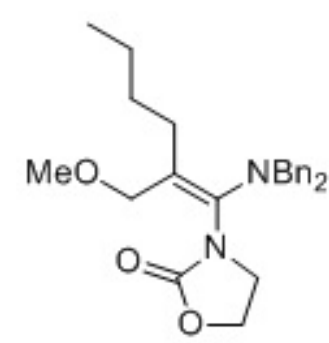

3at, $400 \mathrm{MHz}, \mathrm{DMSO}-d_{6}$

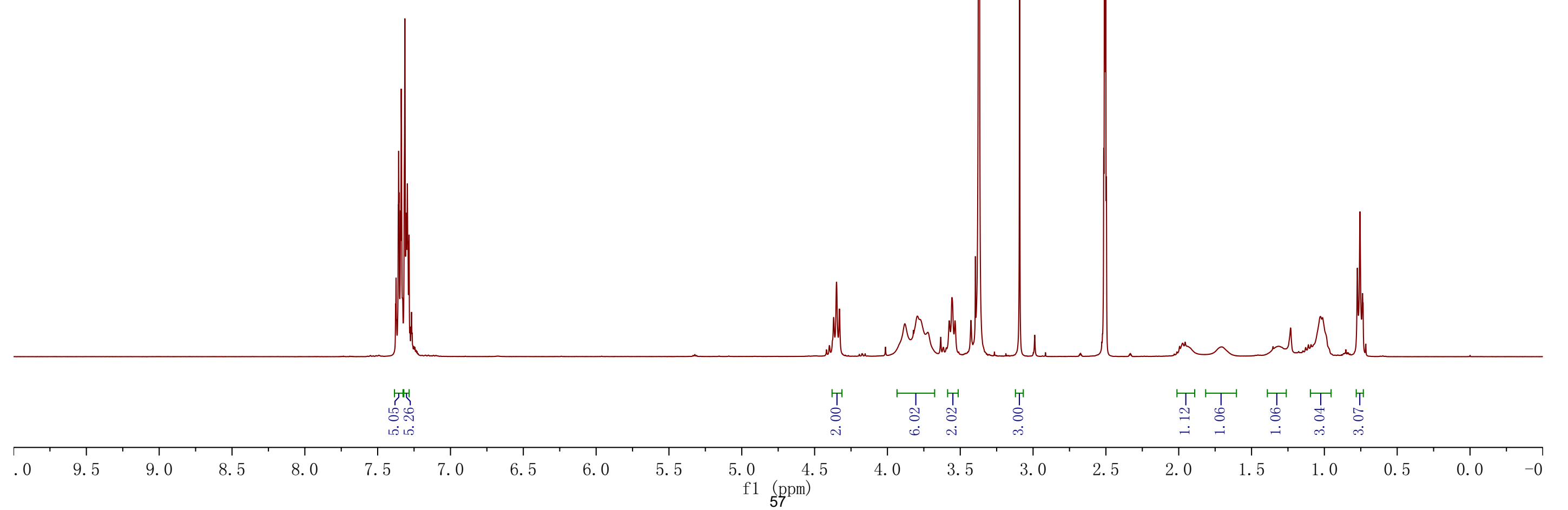




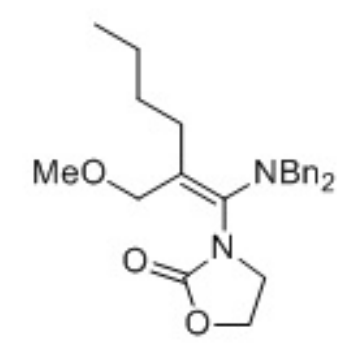

3at, $100 \mathrm{MHz}$, DMSO- $d_{6}$

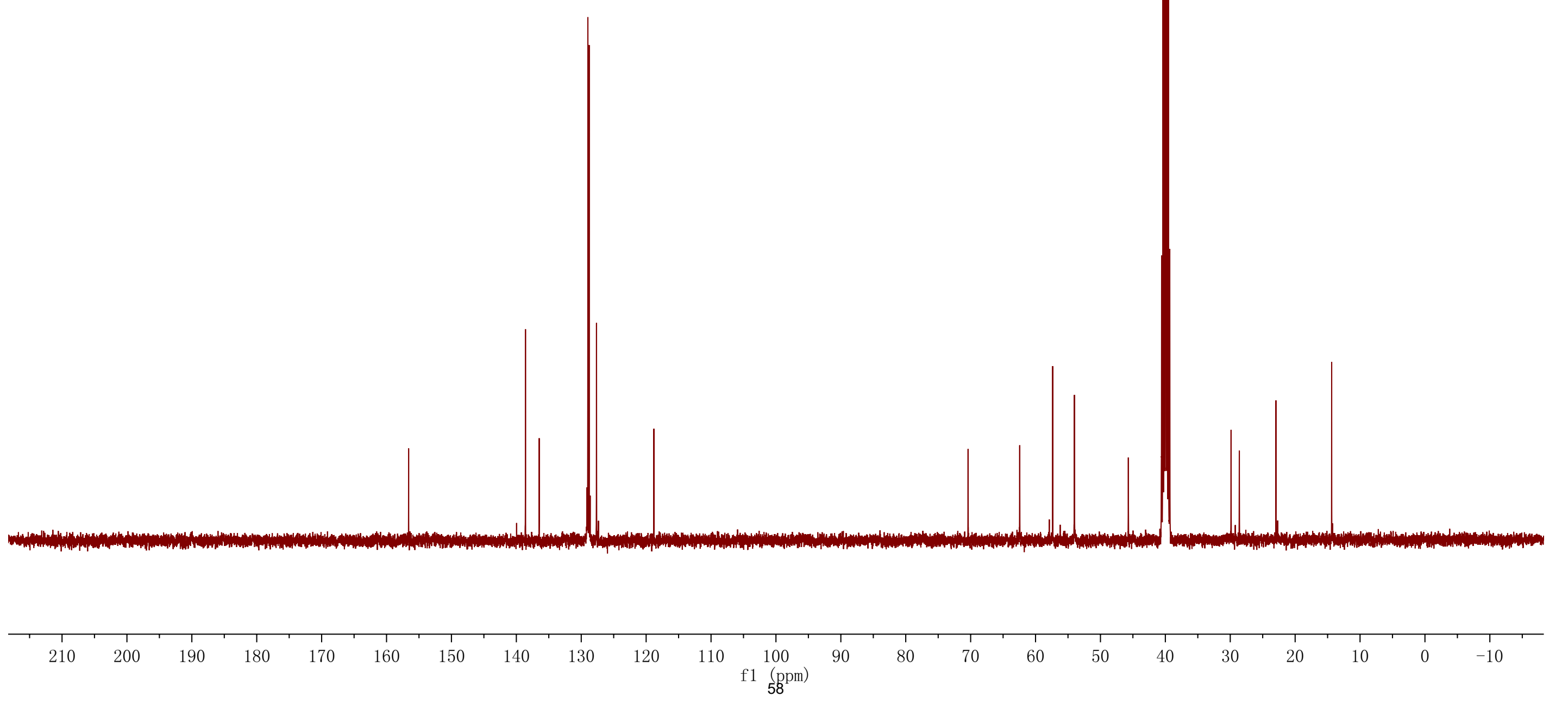




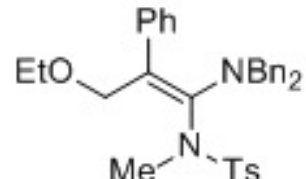

3ba, $400 \mathrm{MHz}$, DMSO- $d_{6}$

\begin{tabular}{|c|c|c|c|c|c|c|c|c|c|c|c|c|c|c|c|c|c|c|c|c|c|c|}
\hline & & & & 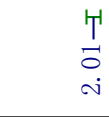 & & & $\begin{array}{l}T \\
+ \\
\dot{0} \\
i\end{array}$ & & & & & & & $\begin{array}{l}T^{\prime} \\
\text { Oे } \\
\dot{i}\end{array}$ & $\begin{array}{l}T^{\prime} T^{\prime} \\
800 \\
\dot{0} 0 \\
\dot{i} \dot{0}\end{array}$ & $\begin{array}{l}T \\
\overrightarrow{0} \\
\dot{s}\end{array}$ & & & $\begin{array}{l}T^{\prime} \\
8 \\
\dot{\infty}\end{array}$ & & & \\
\hline .0 & 9.5 & 9.0 & 8.5 & 8.0 & 7.5 & 7. & & 6.5 & 6.0 & 5.5 & $\begin{array}{r}5.0 \\
\text { f1 }\end{array}$ & pm) & 4. 0 & 3.5 & 3.0 & 2.5 & 2.0 & 1.5 & 1.0 & 0.5 & 0.0 & -0 \\
\hline
\end{tabular}




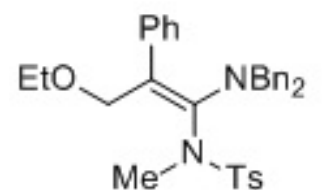

3ba, $75 \mathrm{MHz}, \mathrm{DMSO}-d_{6}$

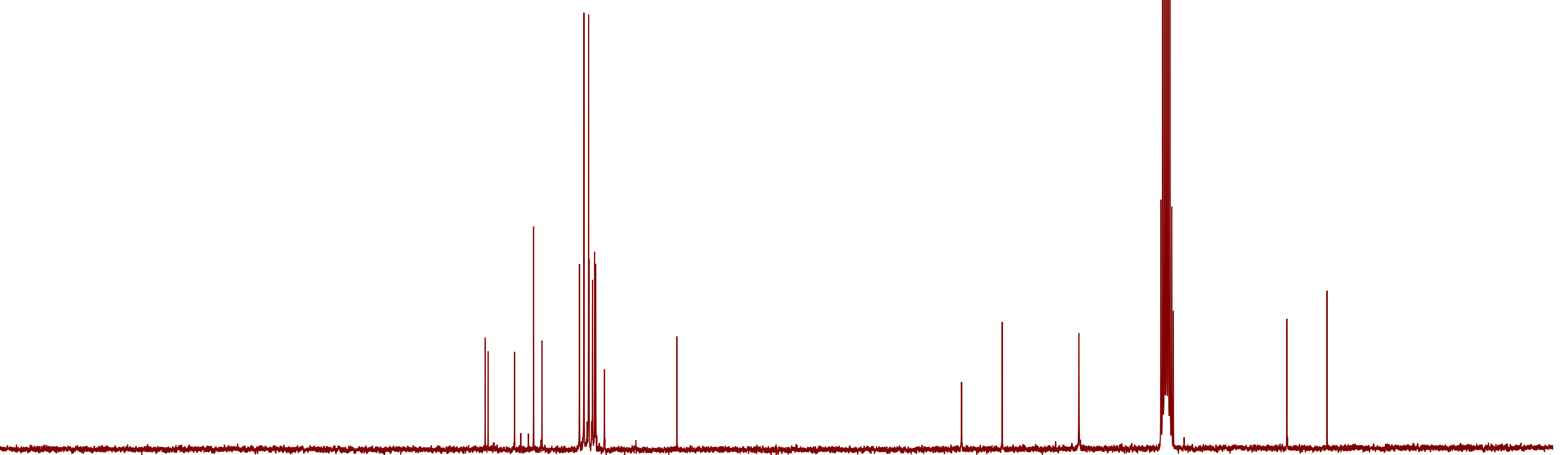

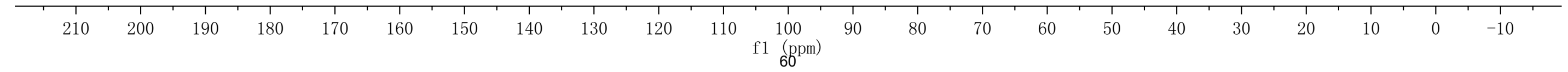




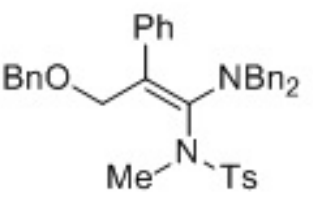

3ca, $400 \mathrm{MHz}, \mathrm{DMSO}-d_{6}$
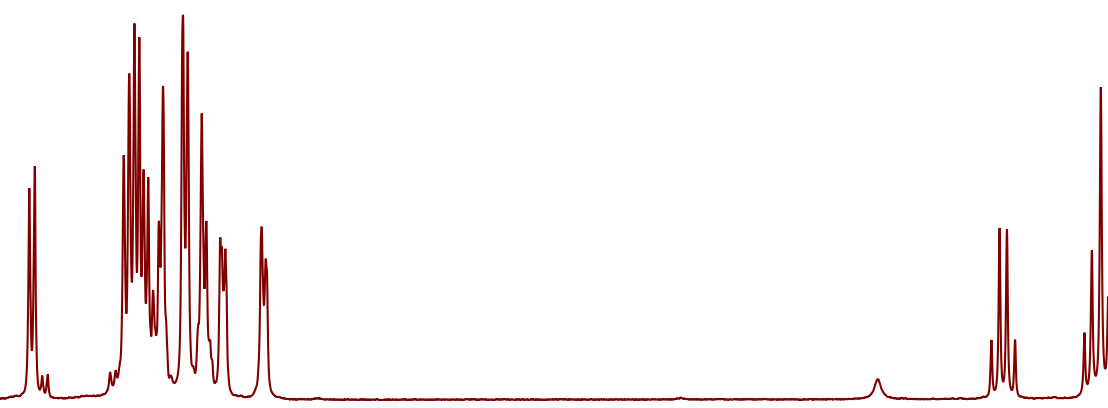

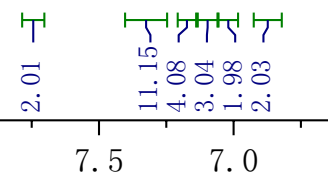




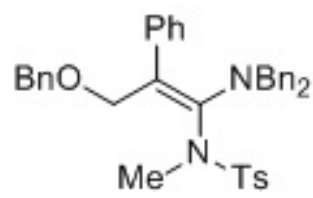

3ca, $75 \mathrm{MHz}, \mathrm{DMSO}-d_{6}$

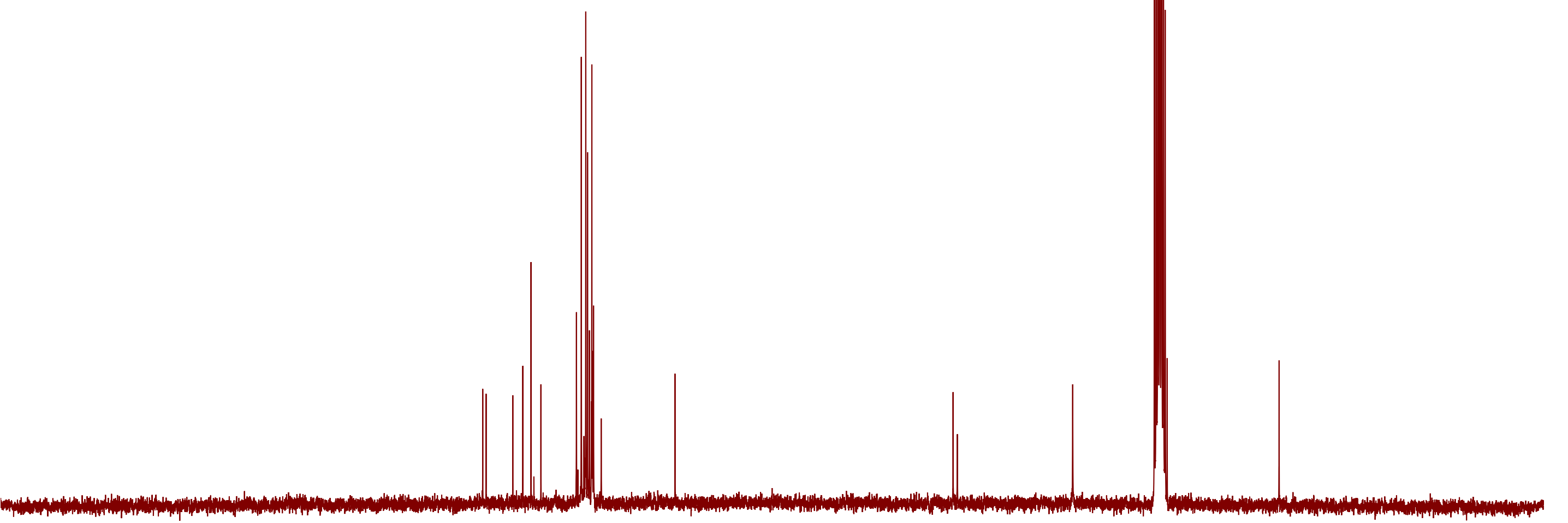

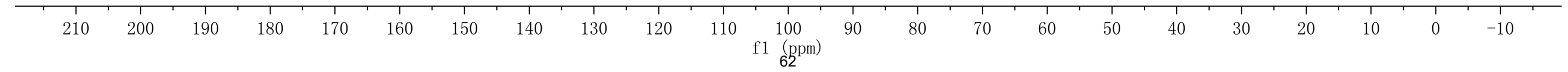




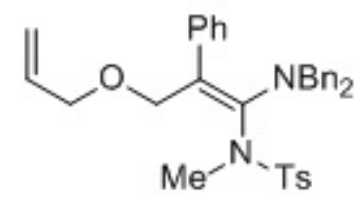

3da, $400 \mathrm{MHz}$, DMSO- $d_{6}$

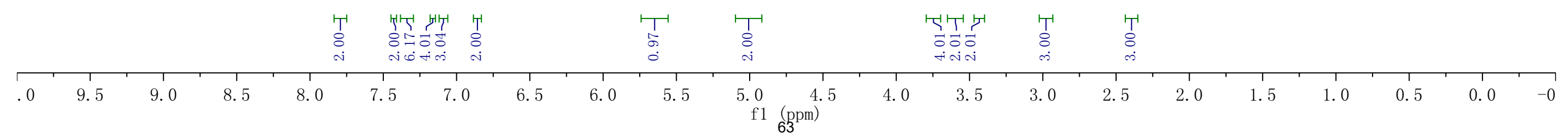




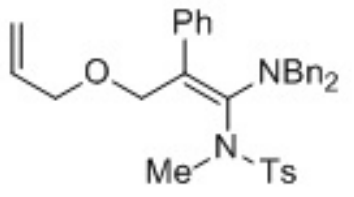

3da, $75 \mathrm{MHz}, \mathrm{DMSO}-d_{6}$

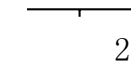




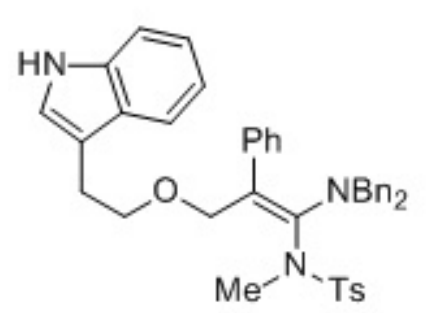

3ea, $400 \mathrm{MHz}$, DMSO- $d_{6}$

\section{$3 \mathrm{ea}, 400 \mathrm{MHz}, \mathrm{DMSO}-d_{6}$}

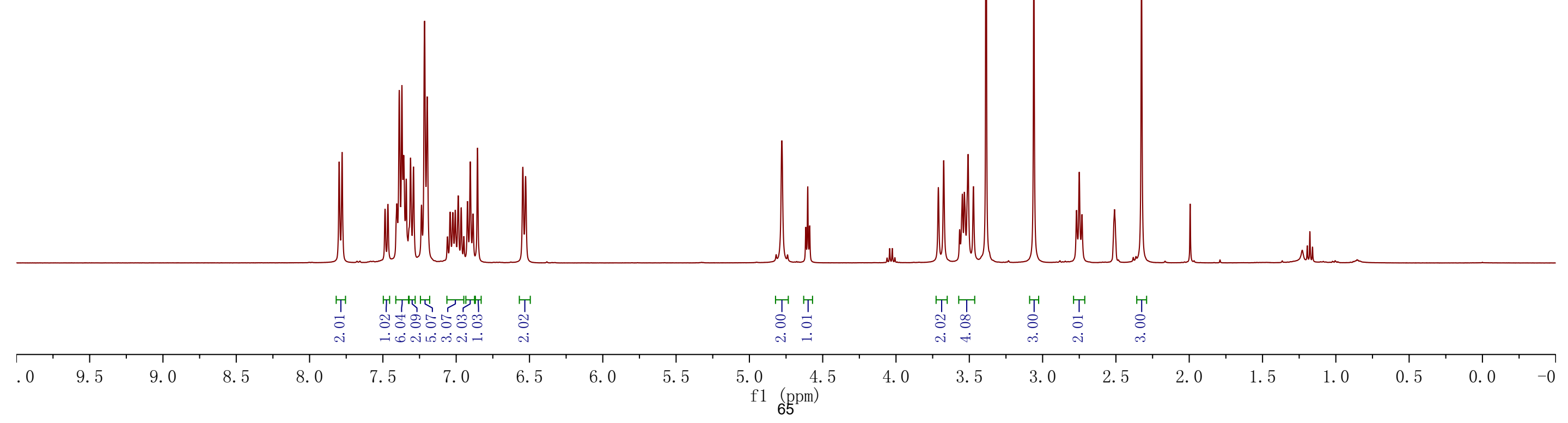




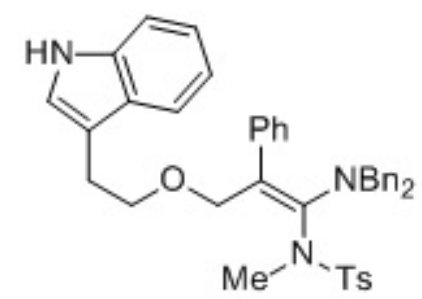

3ea, $100 \mathrm{MHz}, \mathrm{DMSO}-d_{6}$

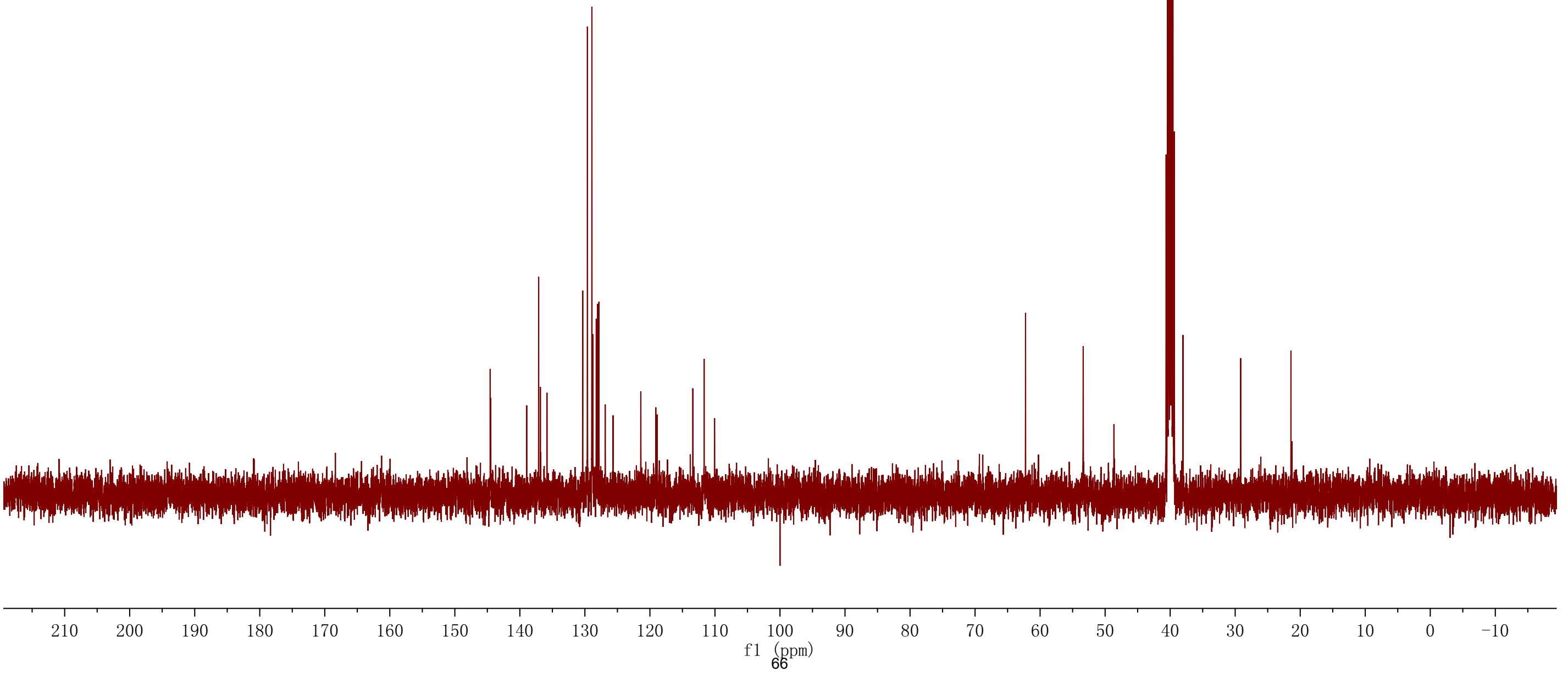




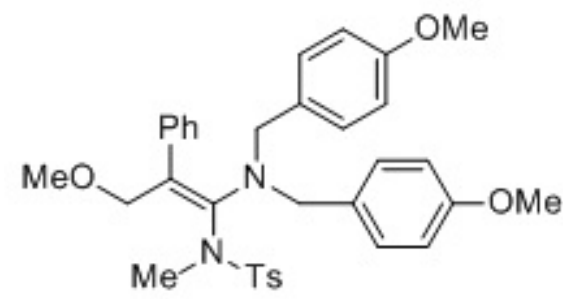

3fa, $400 \mathrm{MHz}, \mathrm{DMSO}_{-} \mathrm{d}_{6}$

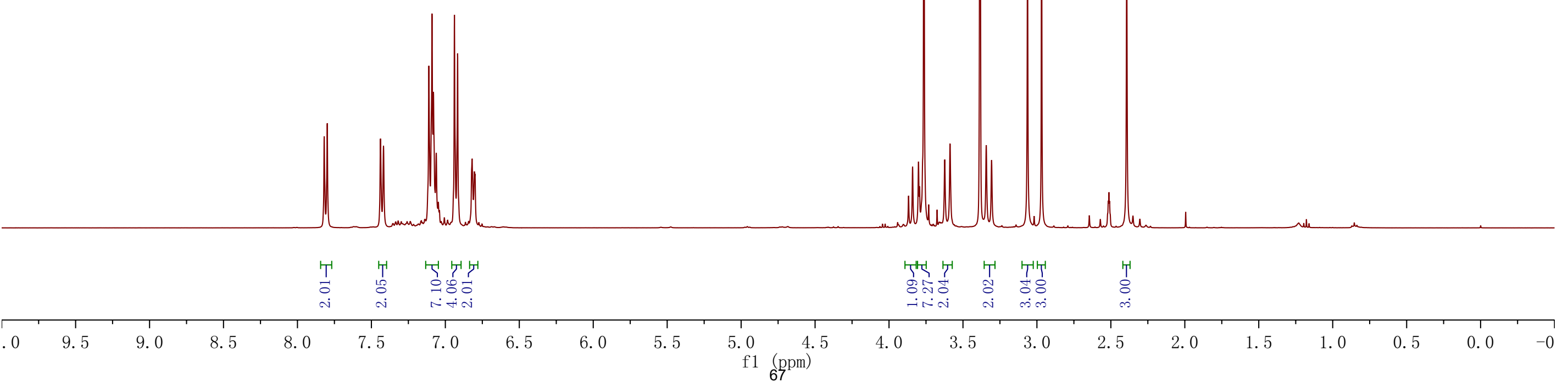




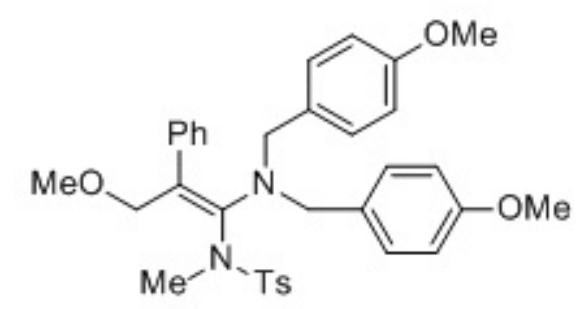

3fa, $100 \mathrm{MHz}$, DMSO- $d_{6}$

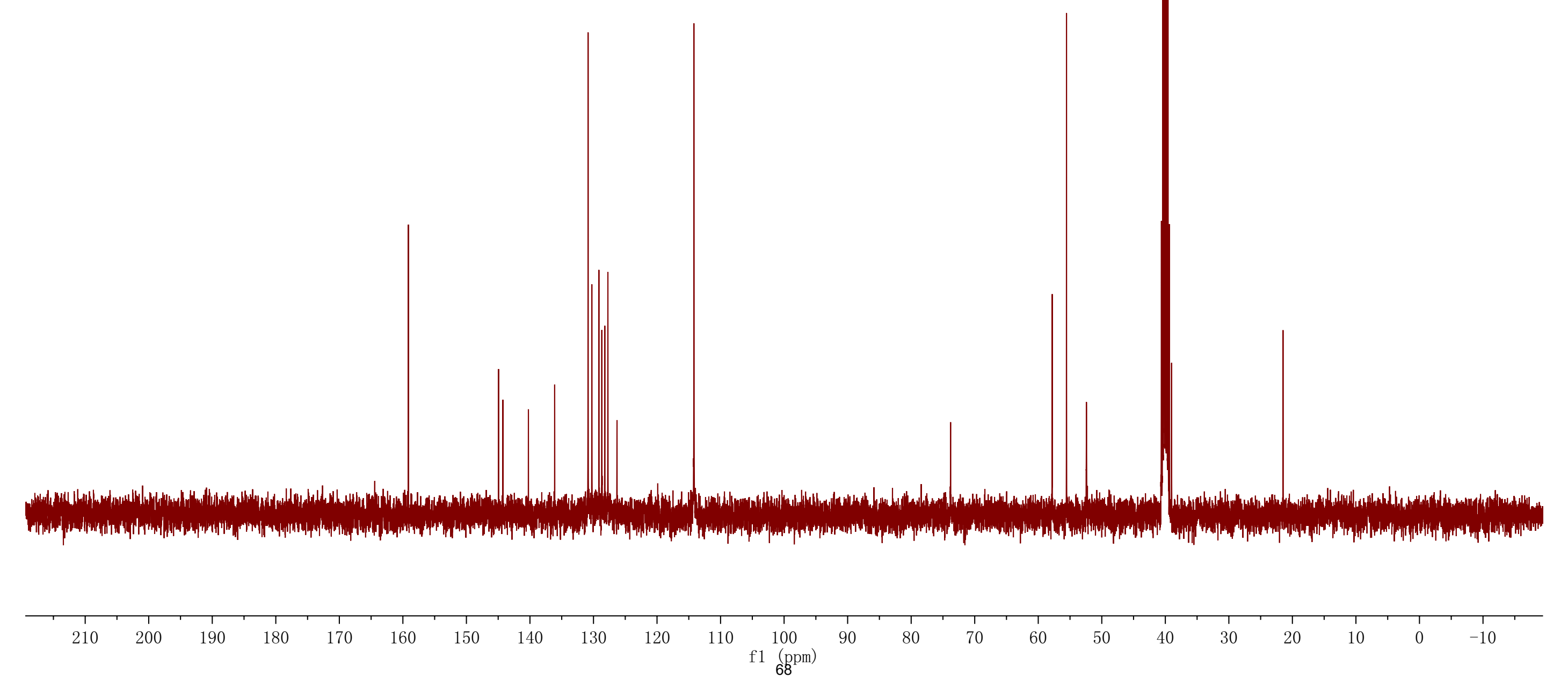




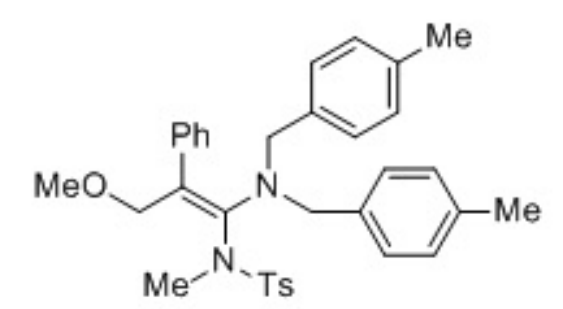

3ga, $400 \mathrm{MHz}$, DMSO- $d_{6}$

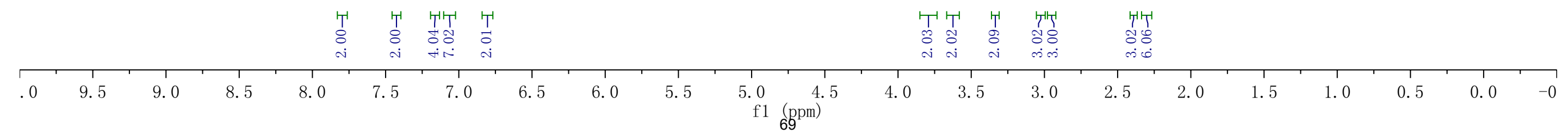




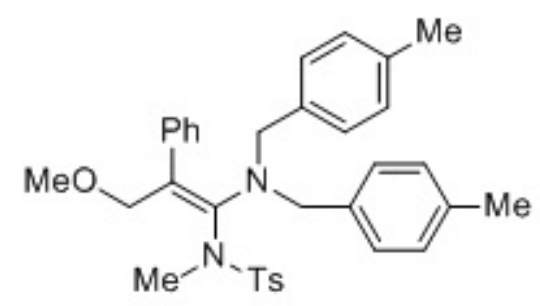

3ga, $75 \mathrm{MHz}, \mathrm{DMSO}-d_{6}$

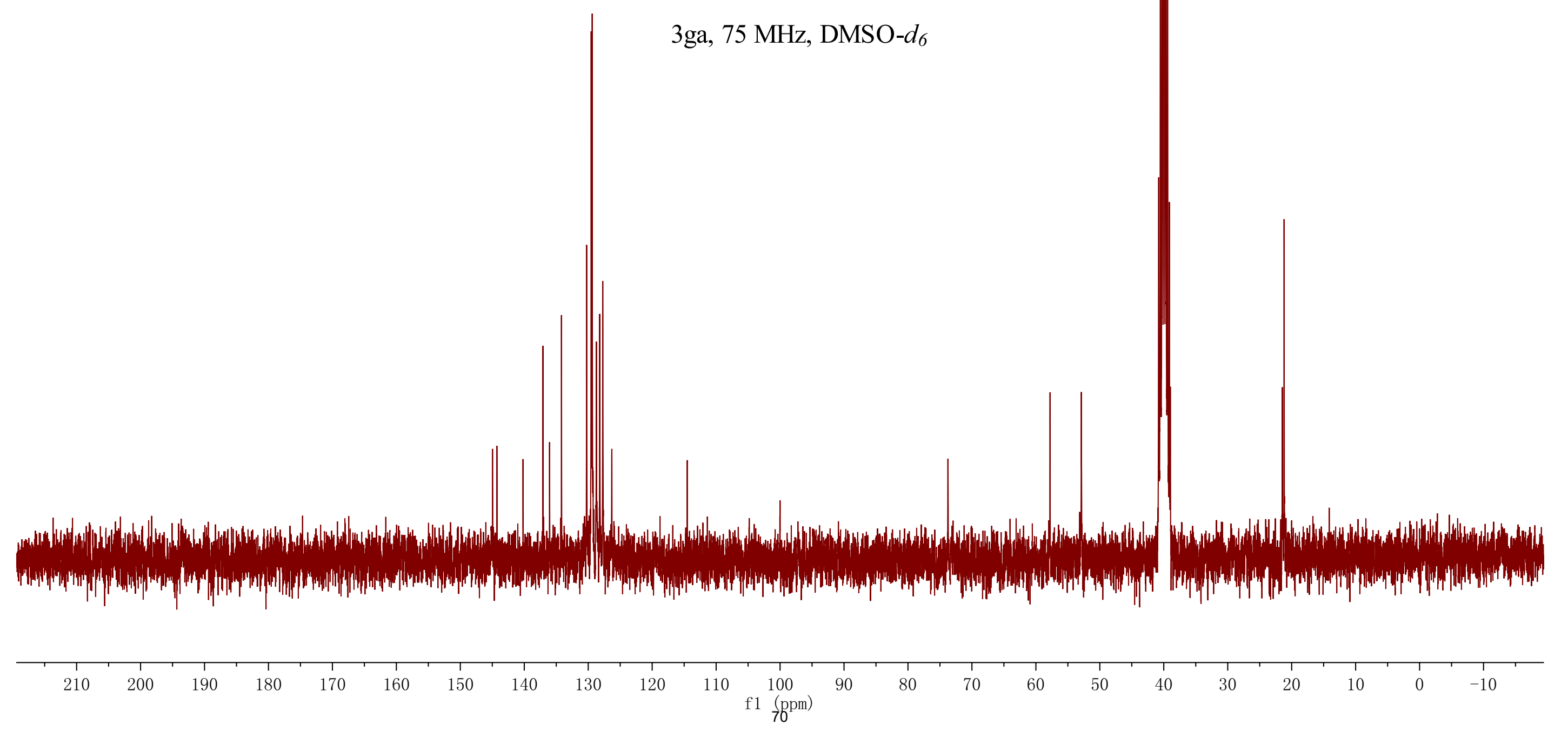




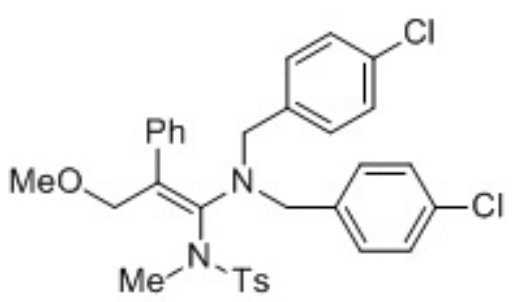

3ha, $400 \mathrm{MHz}$, DMSO- $d_{6}$

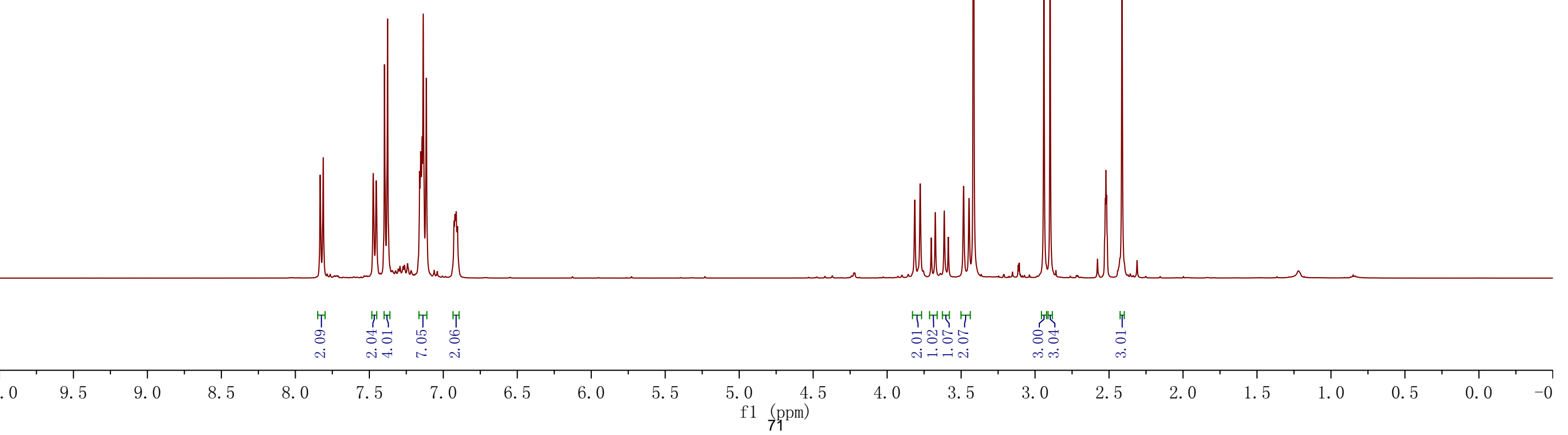


3ha, $100 \mathrm{MHz}$, DMSO- $d_{6}$

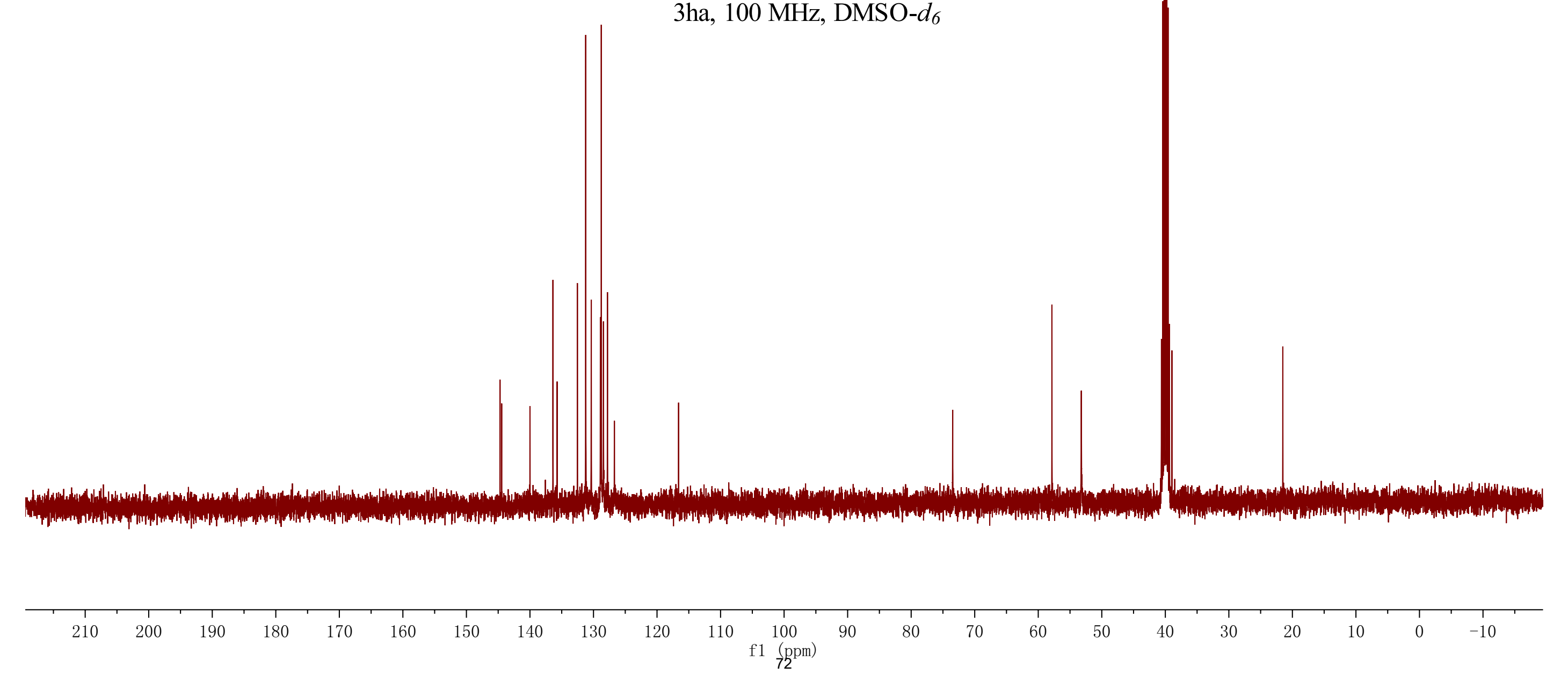


3ia, $400 \mathrm{MHz}, \mathrm{DMSO}-d_{6}$
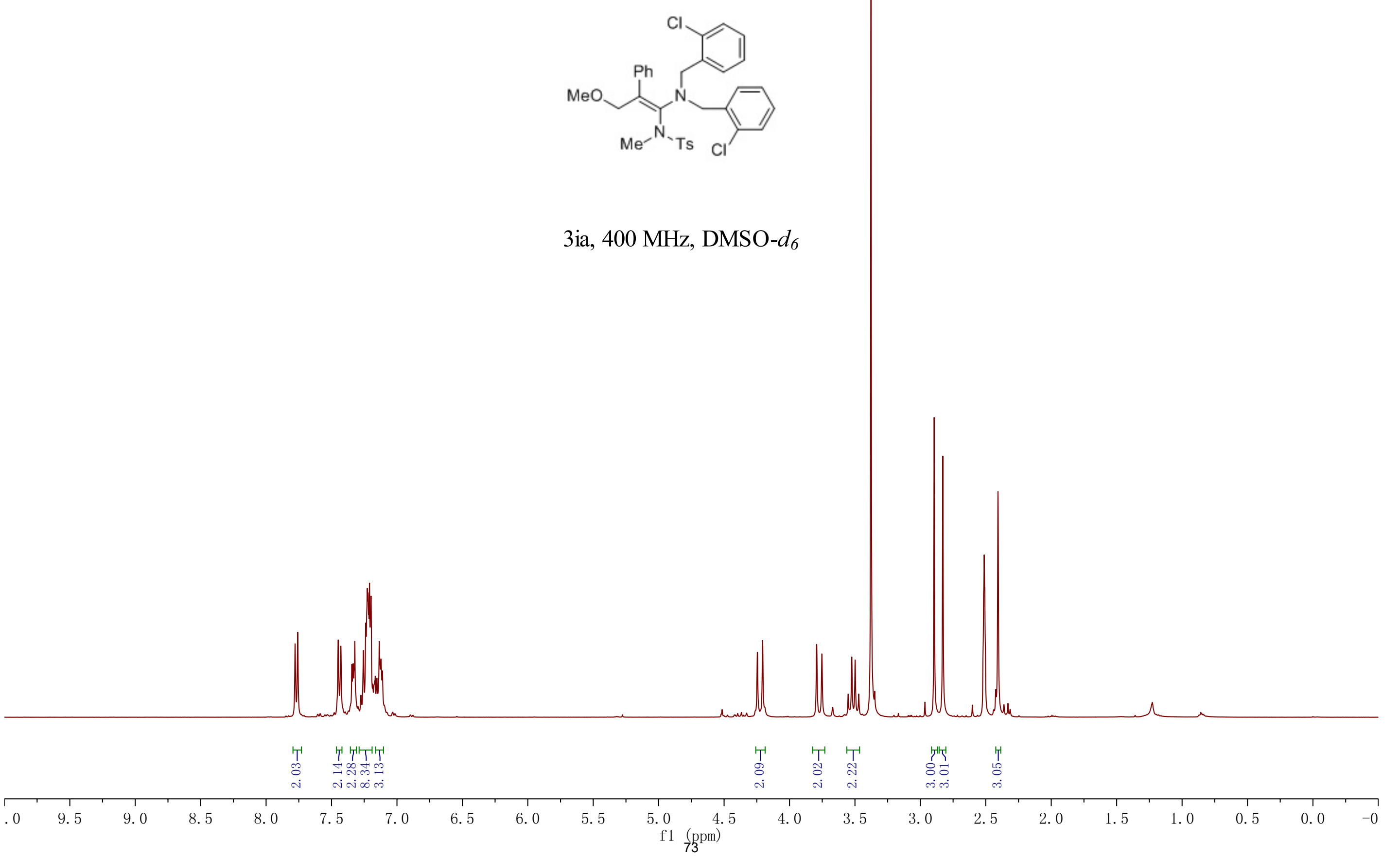
<smiles>COC/C(=C(\N(C)C)N(Cc1ccccc1Cl)Cc1ccccc1Cl)c1ccccc1</smiles>

3ia, $100 \mathrm{MHz}$, DMSO- $d_{6}$

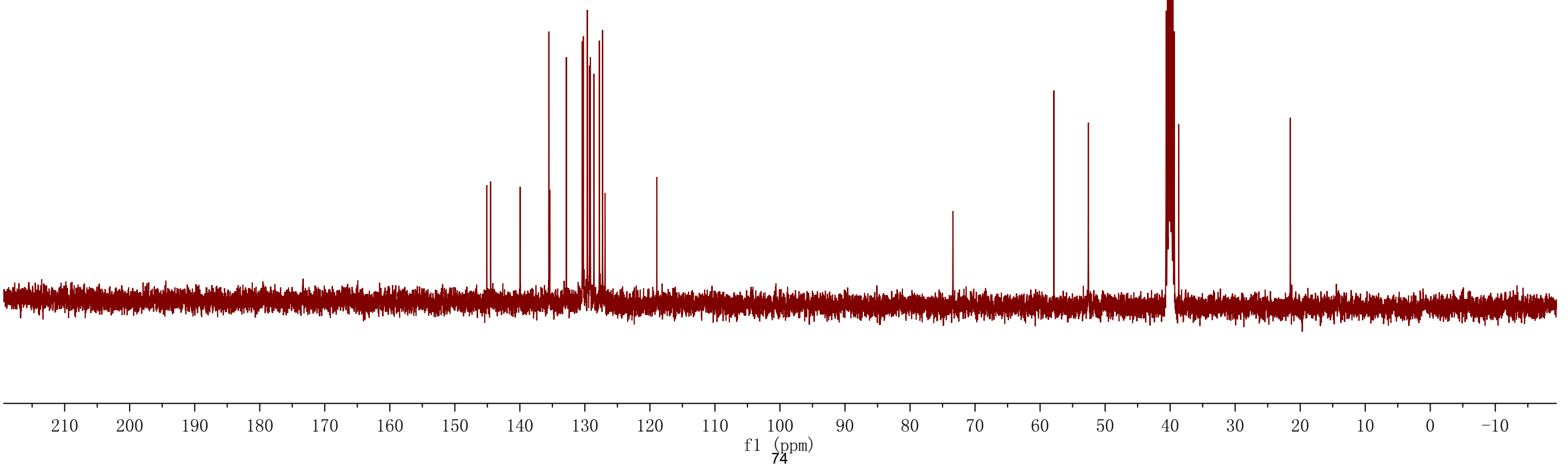




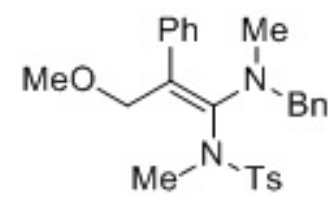

3ja, 400 MHz, DMSO- $d_{6}$

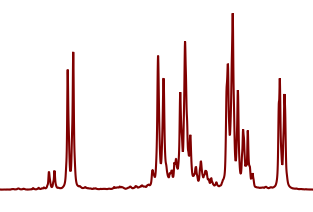
لlut

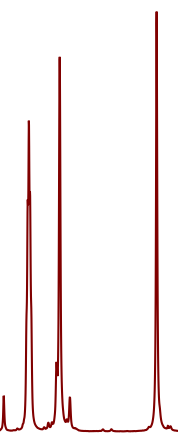

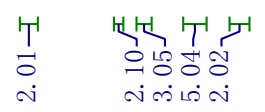

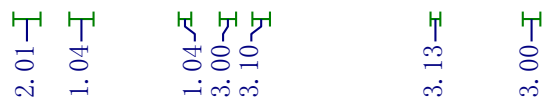




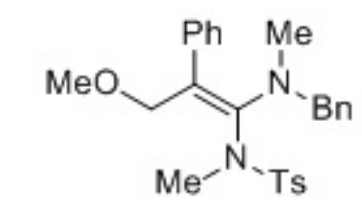

3ja, $100 \mathrm{MHz}$, DMSO- $d_{6}$

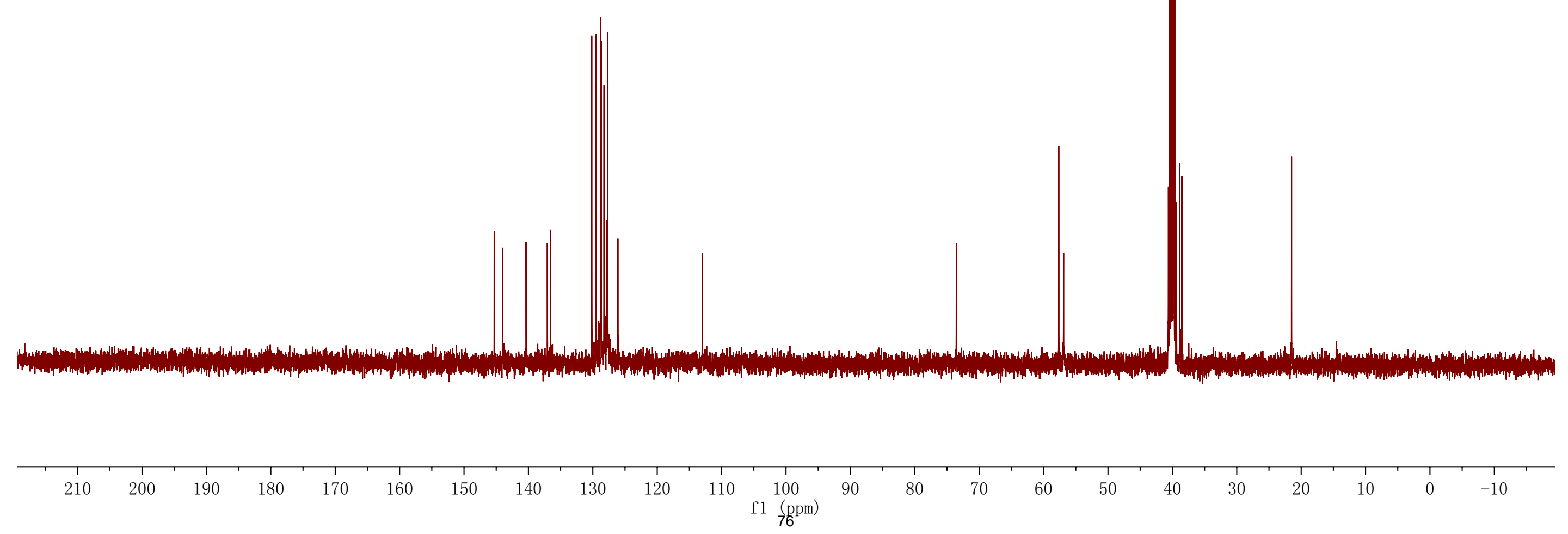




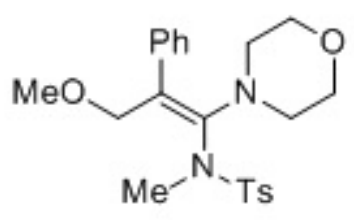

3ka, $400 \mathrm{MHz}, \mathrm{DMSO}-d_{6}$

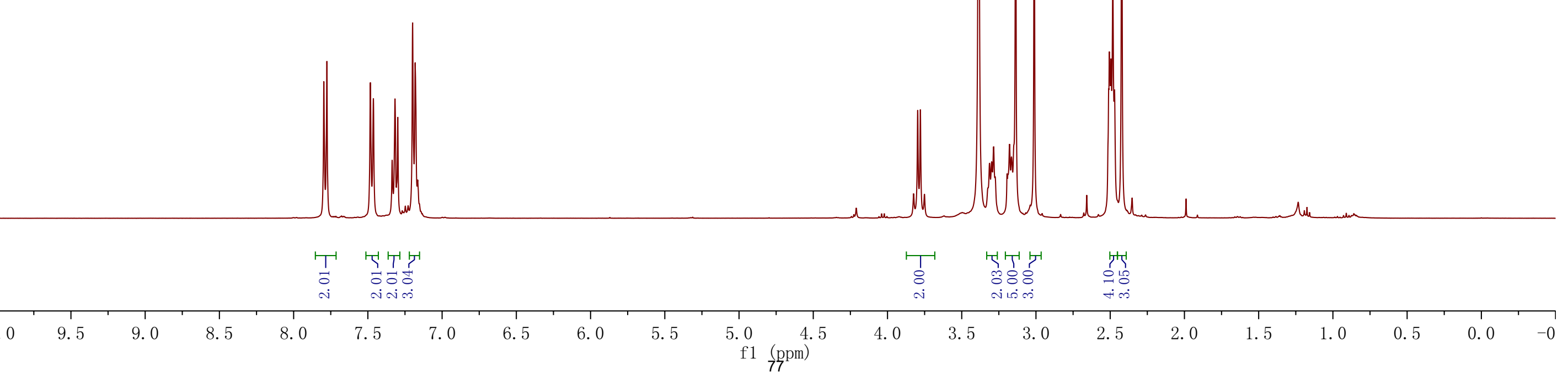




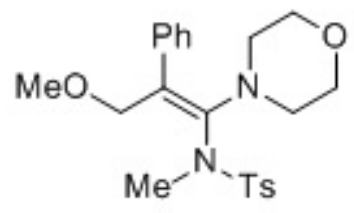

3ka, $100 \mathrm{MHz}$, DMSO- $d_{6}$

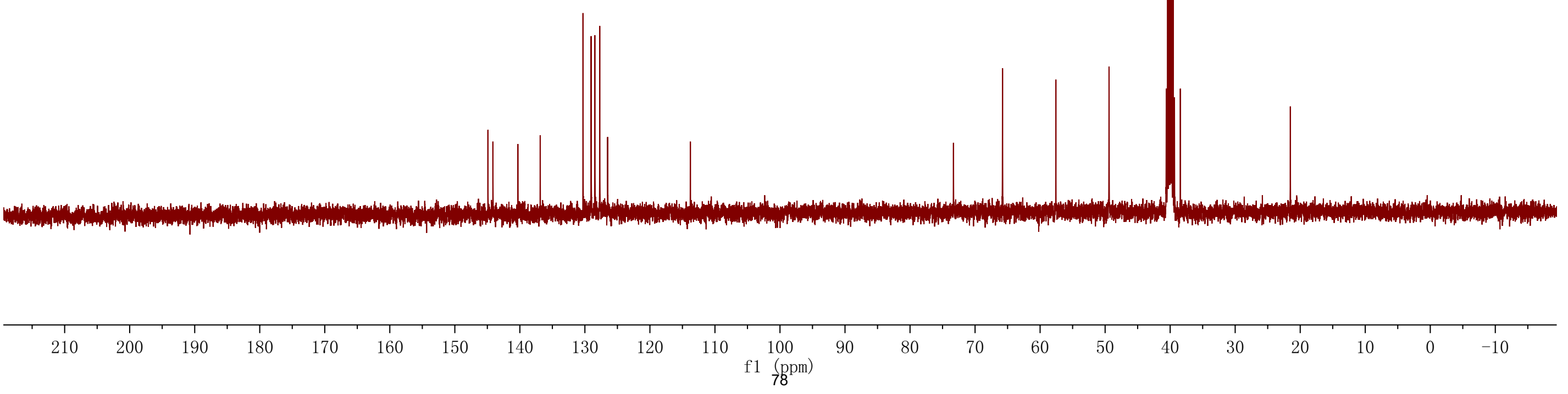




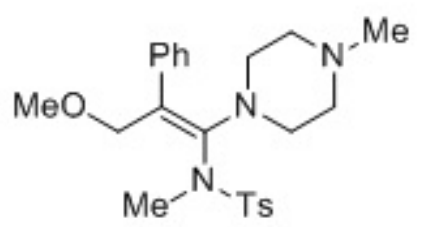

3la, $400 \mathrm{MHz}, \mathrm{DMSO}-d_{6}$

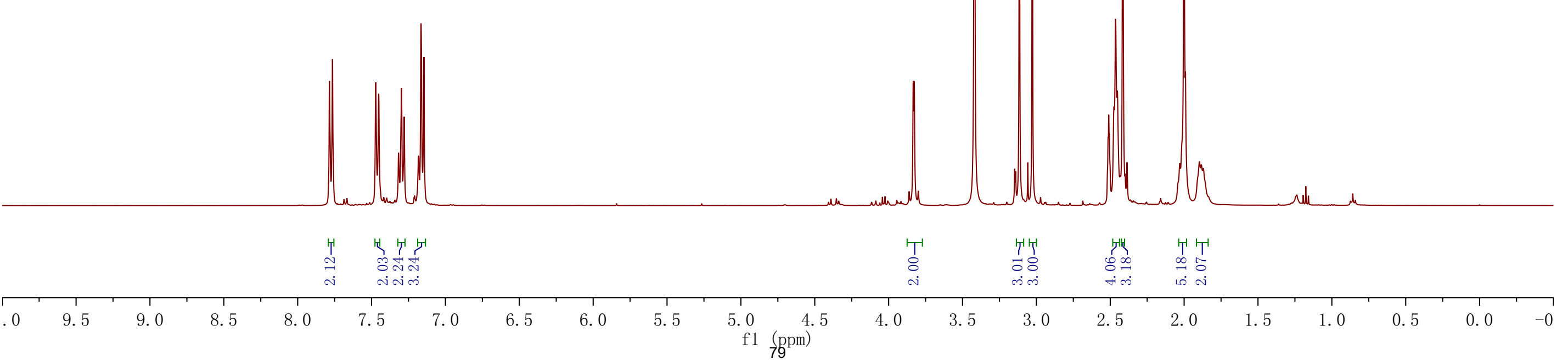




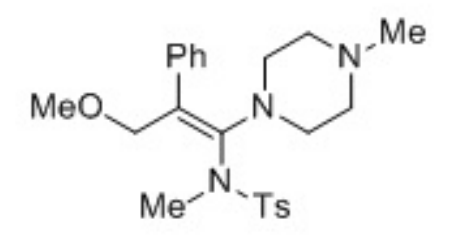

3la, $75 \mathrm{MHz}, \mathrm{DMSO}-d_{6}$

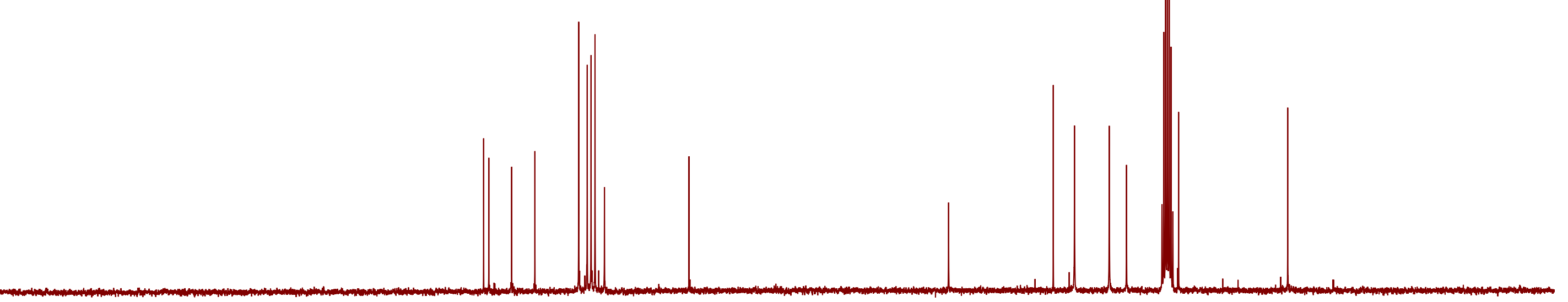

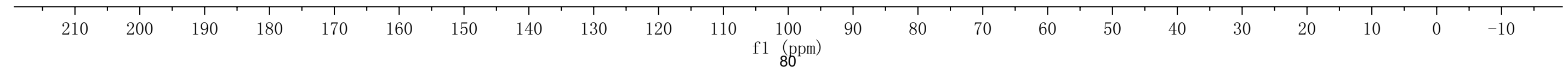




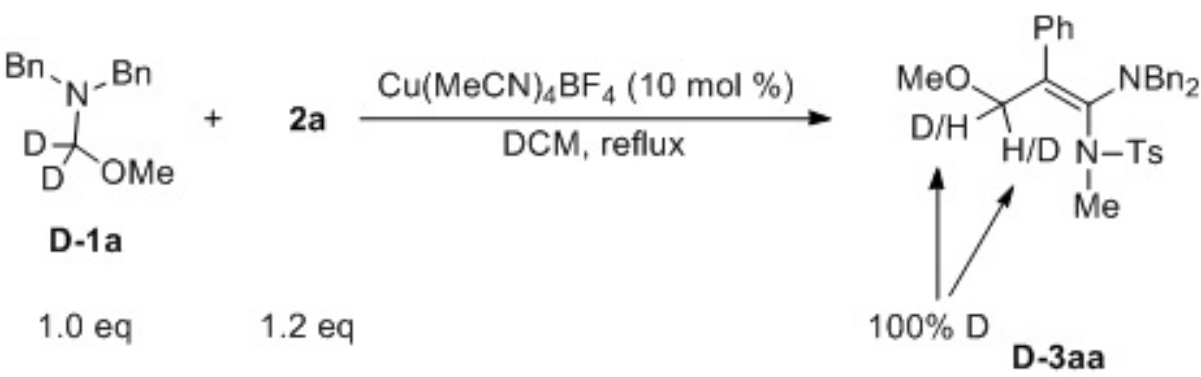

D-3aa, 400 MHz, DMSO-d 6

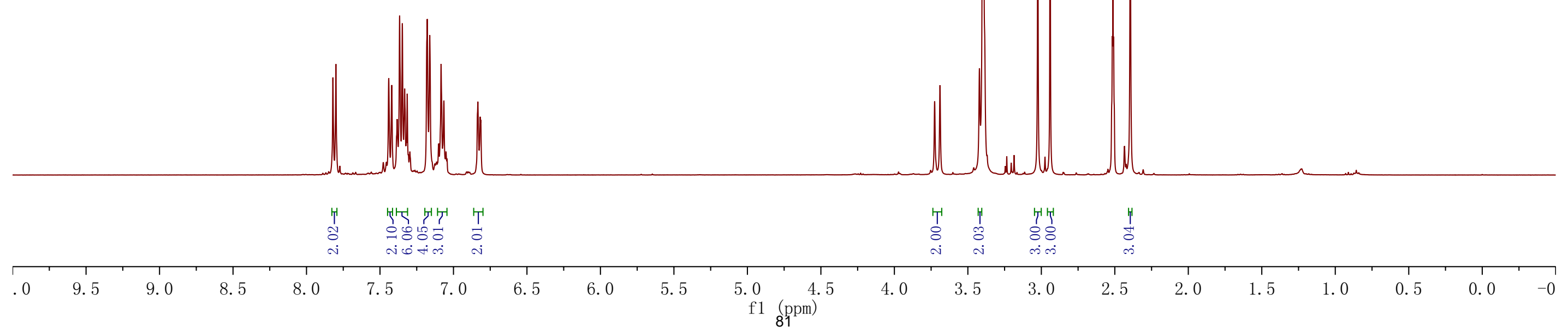




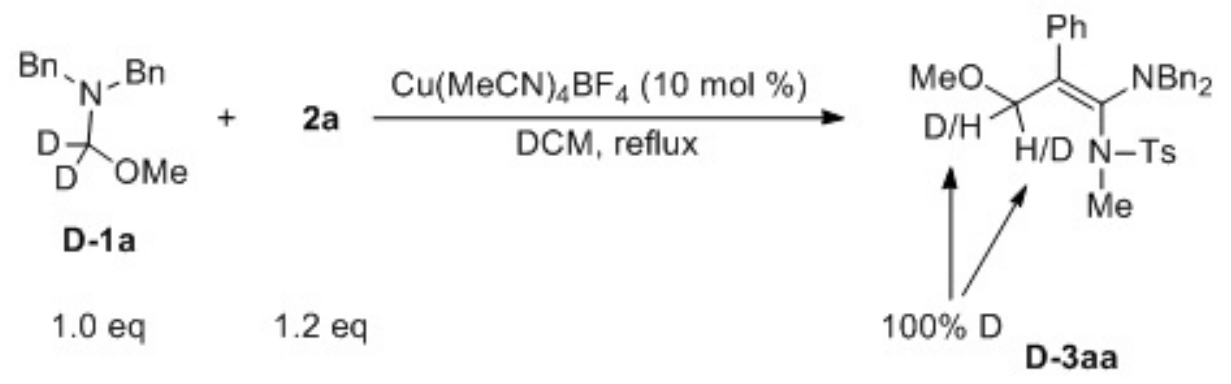

D-3aa, $100 \mathrm{MHz}, \mathrm{DMSO}-d_{6}$

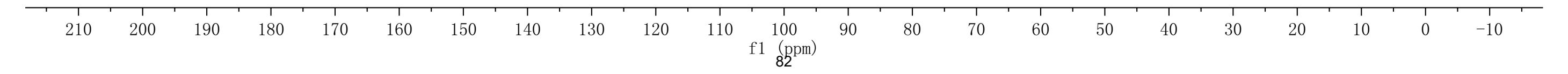




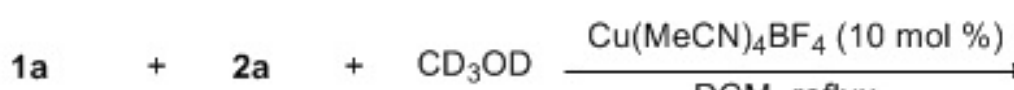

$1.0 \mathrm{eq} \quad 1.2 \mathrm{eq} \quad 1.0 \mathrm{eq}$

DCM, reflux

3aa

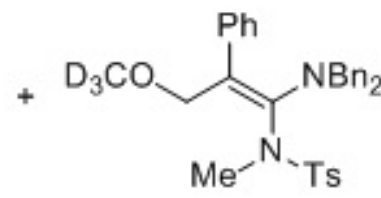

3aa:3aa' = 1:1.14

$400 \mathrm{MHz}, \mathrm{DMSO}-d_{6}$

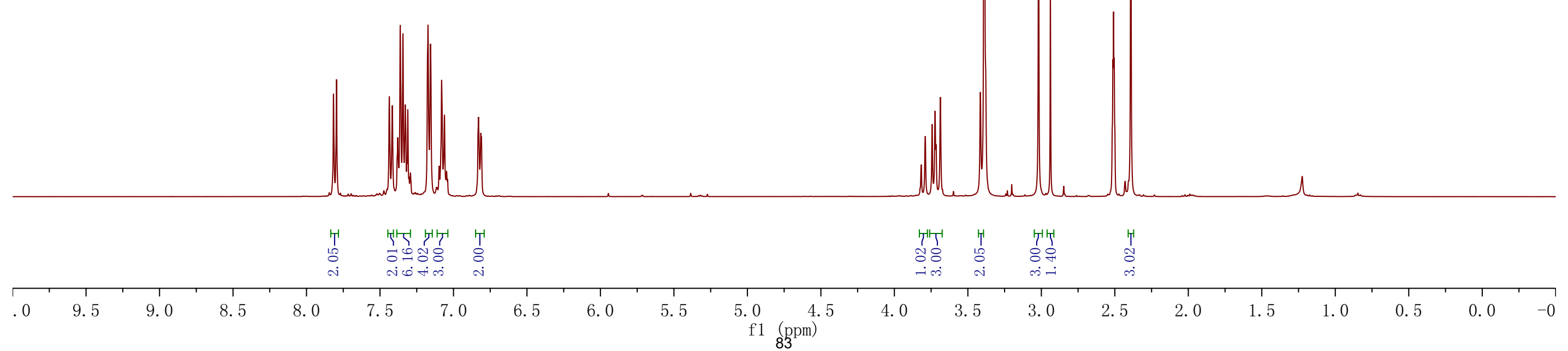




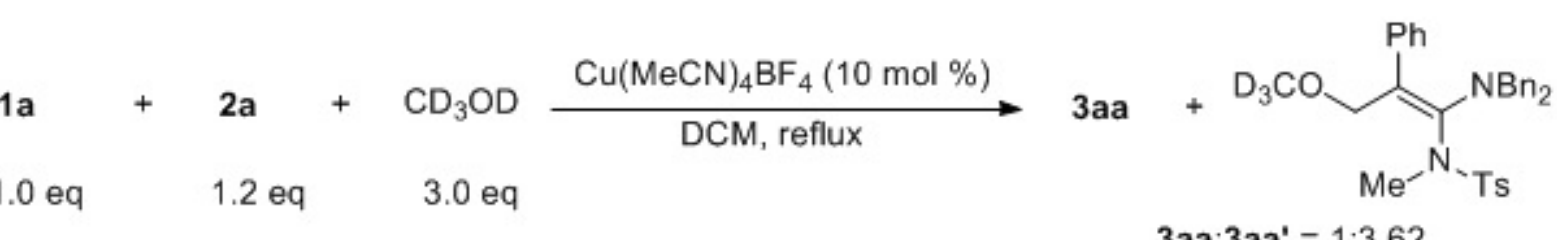

$400 \mathrm{MHz}, \mathrm{DMSO}-d_{6}$

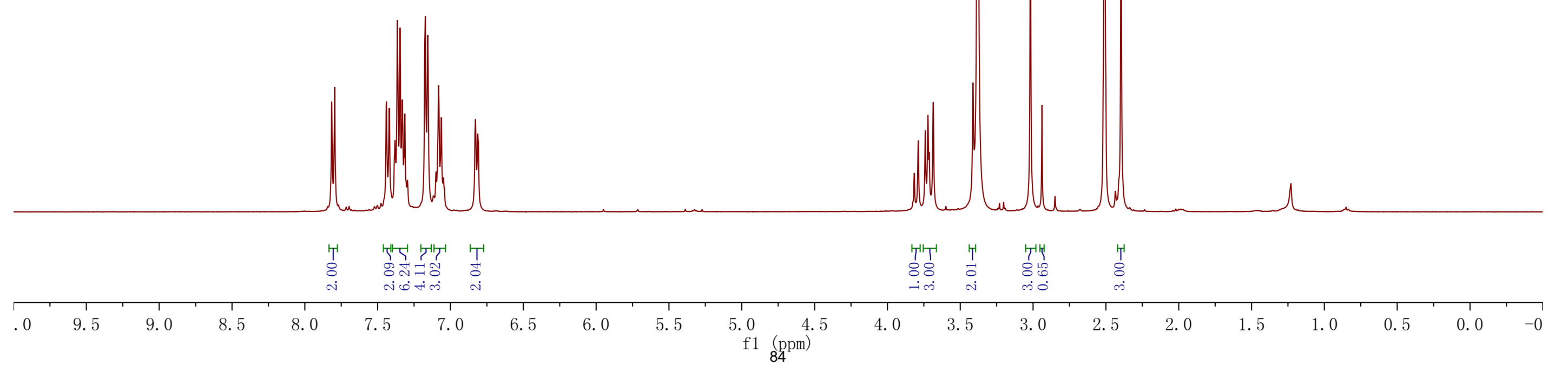




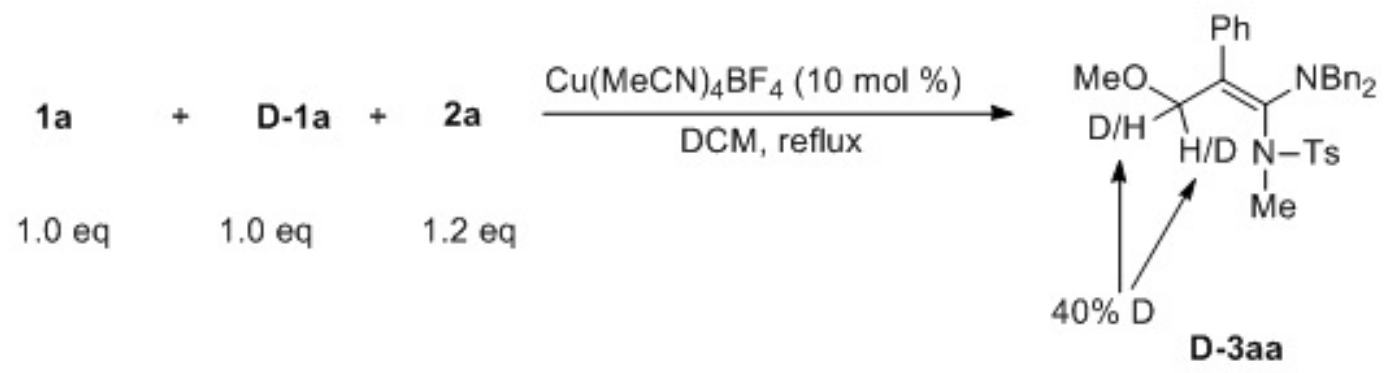

$300 \mathrm{MHz}, \mathrm{DMSO}-d_{6}$

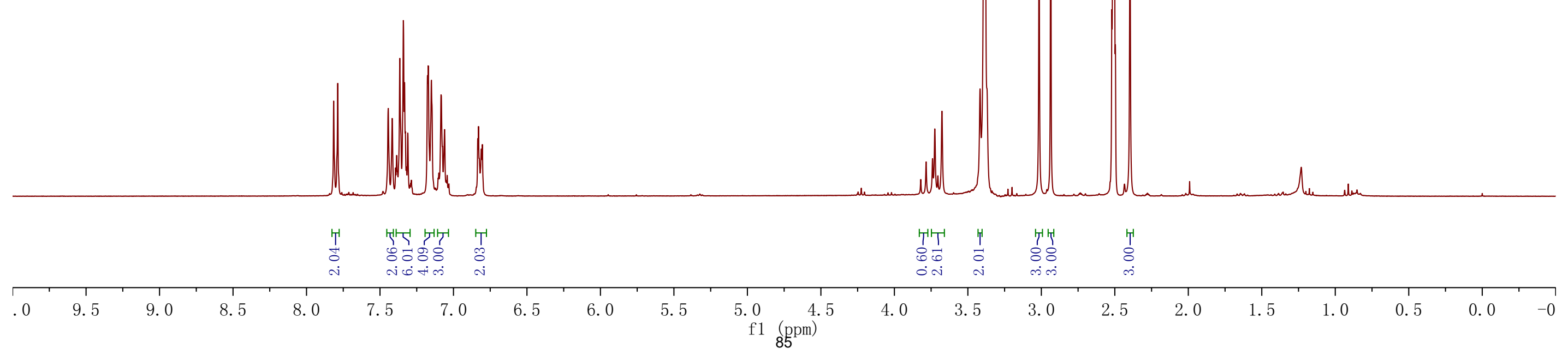




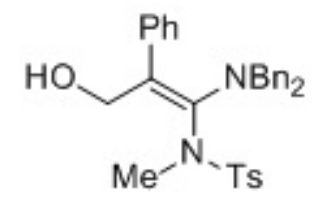

4, $400 \mathrm{MHz}, \mathrm{DMSO}-d_{6}$

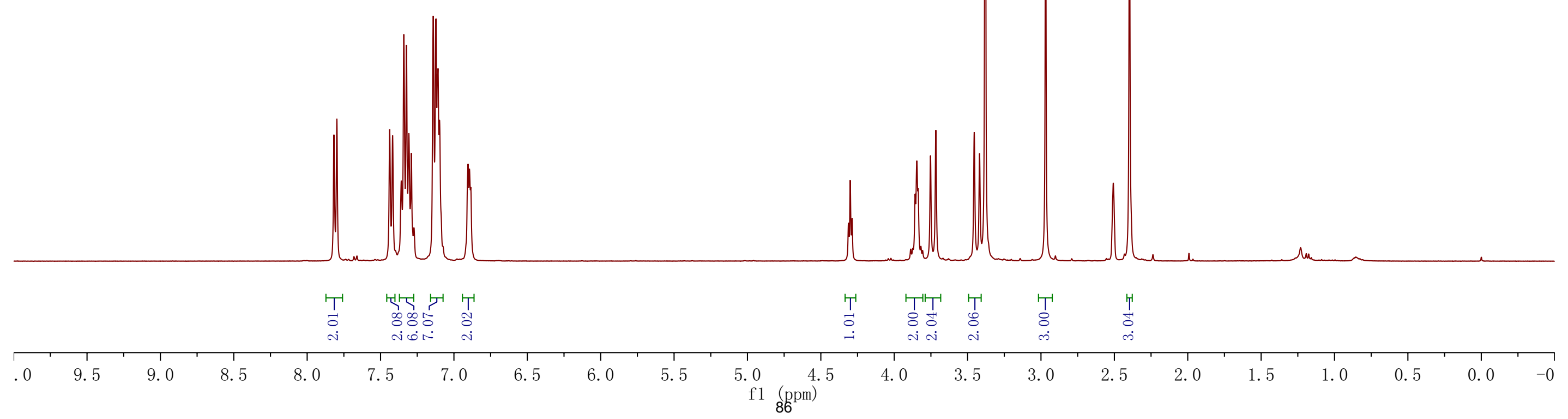




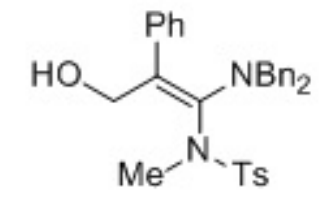

4, $100 \mathrm{MHz}$, DMSO- $d_{6}$
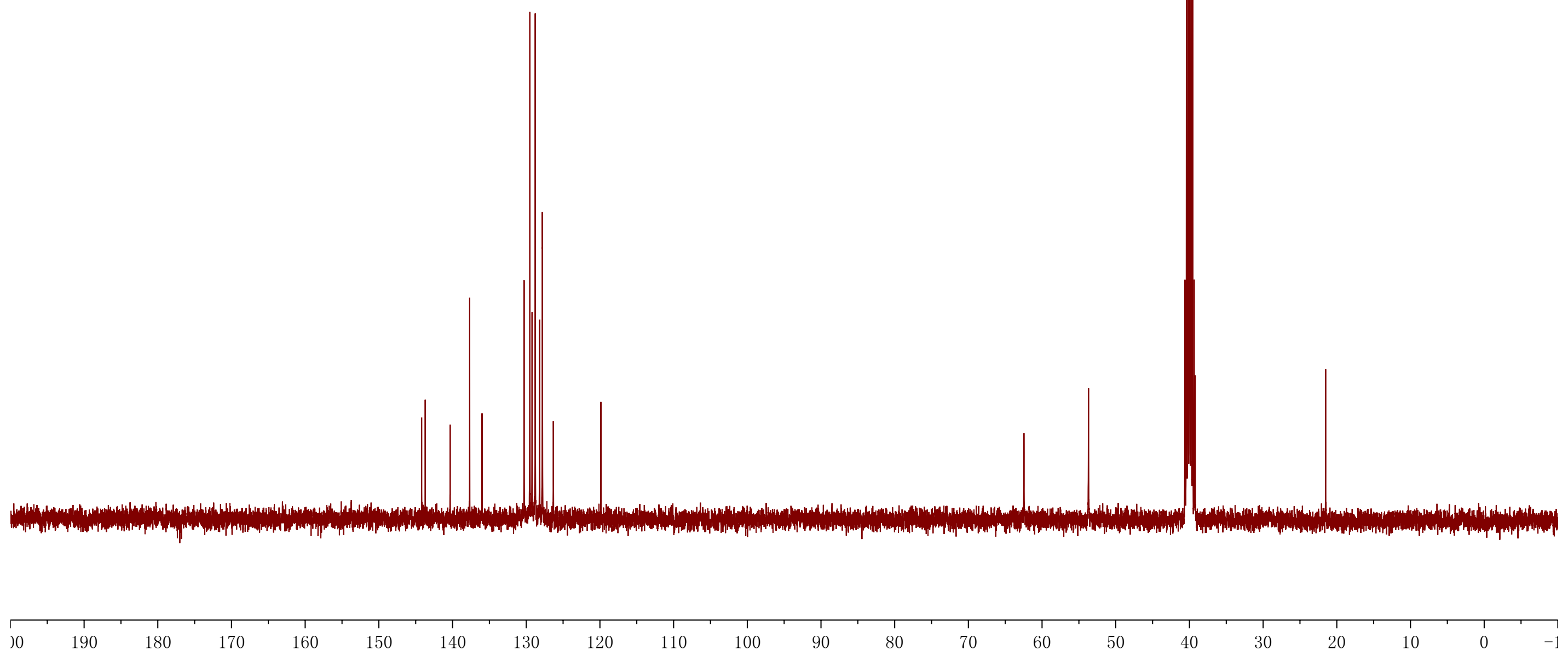

180

170

160

150

140

130

120

110

f1 $87^{(p m)}$ 


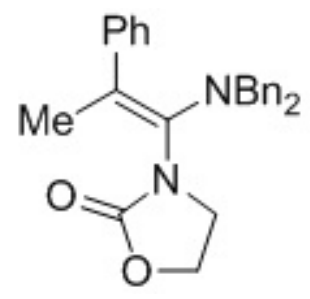

5, $400 \mathrm{MHz}, \mathrm{DMSO}-d_{6}$

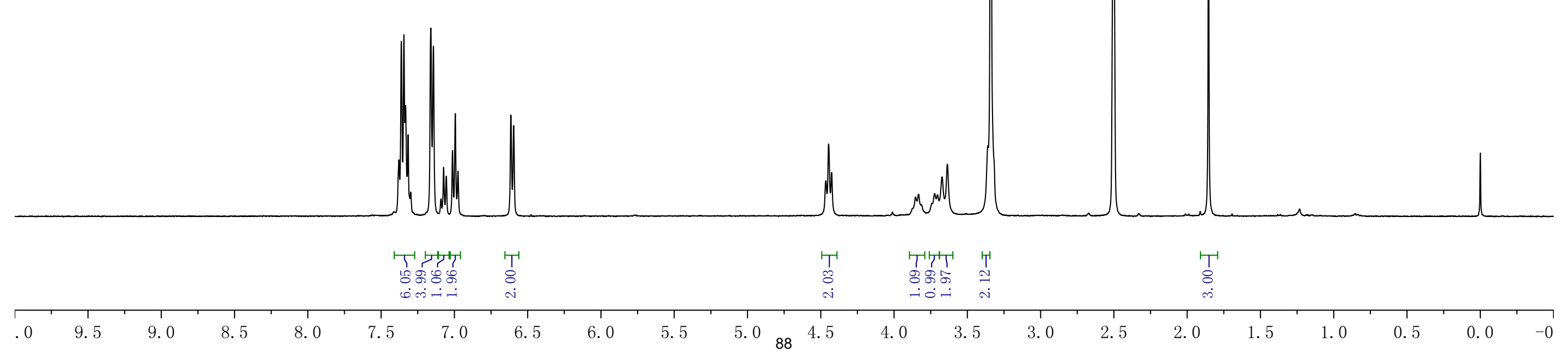


<smiles>CCCCCC(C)=C(CCCC)N1CCOC1=O</smiles>

5, $100 \mathrm{MHz}, \mathrm{DMSO}-d_{6}$ 